\title{
Construções genéricas de espaços de Asplund $C(K)$
}

\author{
Christina Brech
}

TESE APRESENTADA

$\mathrm{AO}$

Instituto de Matemática e Estatística

DA

Universidade De São Paulo

PARA

OBTENÇÃO DO TÍTULO

$\mathrm{DE}$

DOUTOR EM CIÊNCIAS

\author{
Área de Concentração: Matemática \\ Orientador: Prof. Dr. Piotr Koszmider
}

Durante o desenvolvimento deste trabalho o autor recebeu auxílio financeiro da CAPES e do CNPq.

São Paulo, março de 2008 



\title{
Construções genéricas de espaços de Asplund $C(K)$
}

\author{
Este exemplar corresponde à redação \\ final da tese devidamente corrigida \\ e defendida por Christina Brech \\ e aprovada pela Comissão Julgadora.
}

Banca Examinadora:

- Prof. Dr. Piotr Koszmider (orientador) - T.U. Łodz.

- Prof. Dr. Artur Tomita - IME-USP.

- Prof. Dr. Stevo Todorcevic - Université Paris 7.

- Prof. Dr. Jordi Lopez Abad - Université Paris 7.

- Prof. Dr. Jorge Mujica - IMECC-UNICAMP. 

para Papa, Mummy, Ana, Gui e Alexandre 



\section{Agradecimentos}

Em primeiro lugar, tenho que agradecer aos meus orientadores Piotr Koszmider e Stevo Todorcevic. O Piotr, cuja paixão pela matemática foi uma fonte de motivação para meu trabalho, me introduziu às aplicações de teoria dos conjuntos à teoria dos espaços de Banach e me incentivou a seguir nesta linha no doutorado e a fazer um estágio de pesquisa em Paris com o Stevo. Foi sempre atencioso, paciente e exigente. O Stevo aceitou ser meu coorientador e agradeço-lhe por sua disponibilidade e por ter me explicado inúmeras vezes coisas que lhe pareciam tão óbvias e a mim, tão improváveis. Seria impossível resumir em algumas linhas toda a gratidão que sinto por eles: aos dois, muito obrigada!

Agradeço aos Profs. Joan Bagaria e Jorge Mujica por terem aceitado fazer um parecer desta tese, apesar do curto prazo que lhes foi proposto. Agradeço igualmente ao Prof. Artur Tomita, que aceitou participar da banca, e mais particularmente ao Prof. Jordi Lopez Abad, por sua participação na banca, sua ajuda na minha chegada a Paris e seu interesse pelo meu trabalho.

Todo o meu reconhecimento às Profas. Zara Issa Abud e Mary Lilian Lourenço, que guiaram meus primeiros passos na carreira de matemática. Agradeço sinceramente a elas e também aos outros matemáticos do IME e de Chevaleret que me ajudaram ao longo do caminho. As conversas com Ofelia Alas, Antonio Avilés, Valentin Ferenczi, Gilles Godefroy e Lúcia Junqueira me permitiram melhor compreender alguns mistérios da topologia e da teoria dos espaços de Banach.

Finalmente, agradeço aos meus colegas (ex-)doutorandos que tiveram a paciência de ouvir minhas queixas de doutoranda angustiada e com quem tive a oportunidade de travar boas discussões ao longo dos últimos anos. O grupo brasileiro, especialmente Alexandre, André 
Hallack, Armando, Demo, Leo, Marcelo Major, Marina, Natalia, Pavlos, Pedro, Rogério e Samuel. E a trupe francesa, que me acolheu calorosamente na sala 5C06, começando por Avenilde e Fares, mas sem esquecer Adrien, Caroline, Clément, David, Elizabeth, Gönenç, Karim, Lionel, Luís, Martin, Matteo e Victor.

Sandro et Valentin, merci pour la révision du texte français! 
Odkrycie Banacha byto moim największym odkryciem naukowym.

(Hugo Steinhaus) 



\section{Resumo}

Neste trabalho consideramos um método de construções genéricas de espaços compactos e dispersos não-metrizáveis, desenvolvido por Baumgartner, Shelah, Rabus, Juhasz e Soukup. Introduzimos novas técnicas e obtemos novas aplicações relevantes tanto para a topologia dos espaços compactos quanto para a geometria dos espaços de Banach de funções contínuas.

As novas técnicas dizem respeito a novas amalgamações de condições do forcing que adiciona os espaços dispersos, bem como a generalizações dos argumentos dos autores acima citados de pontos de um espaço compacto $K$ para medidas de Radon sobre $K$.

Como aplicações, obtemos dois novos espaços compactos e dispersos $K_{1}$ e $K_{2}$, com as propriedades abaixo.

$K_{1}$ é um espaço hereditariamente separável de peso $\aleph_{1}$ tal que $C\left(K_{1}\right)$ possui a propriedade (C) de Corson e não possui a propriedade (E) de Efremov.

$K_{2}$ é o primeiro exemplo de um espaço compacto disperso, hereditariamente separável, de altura $\omega_{2}$. Segue que o grau de Lindelöf hereditário de $K_{2}$ é $\aleph_{2}$, mostrando a consistência de $h L(K) \not \leq h d(K)^{+}$para espaços compactos $K . C\left(K_{2}\right)$ é o primeiro exemplo consistente de um espaço de densidade $\aleph_{2}$ que não possui um sistema biortogonal não-enumerável.

Palavras-chave: espaço de Banach $C(K)$, forcing, espaço disperso, espaço hereditariamente separável, espaço de Asplund, renormações, sistema biortogonal. 



\section{Abstract}

In this work we consider a method of generic constructions of compact scattered nonmetrizable spaces developed by Baumgartner, Shelah, Rabus, Juhasz and Soukup. We introduce new techniques and obtain new applications both relevant to topology of compact spaces and the geometry of Banach spaces of continuous functions.

The new techniques concern new amalgamations of conditions of forcing which add the dispersed spaces as well as the generalizations of arguments of the above-mentioned authors from points of a compact space $\mathrm{K}$ to Radon measures on $\mathrm{K}$.

As applications we obtain two compact scattered spaces $K_{1}$ and $K_{2}$ with the properties below.

$K_{1}$ is a hereditarily separable space of weight $\aleph_{1}$ such that $C\left(K_{1}\right)$ has property (C) of Corson and does not have property (E) of Efremov.

$K_{2}$ is the first (consistent) example of a compact scattered space which is hereditarily separable and whose height is $\omega_{2}$. It follows that its hereditary Lindelöf degree is $\aleph_{2}$, showing the consistency of $h L(K) \not L h d(K)^{+}$for compact spaces $K . C\left(K_{2}\right)$ is the first consistent example of a Banach space of density $\aleph_{2}$ without uncountable biorthogonal systems.

Keywords: Banach space $C(K)$, forcing, scattered space, hereditarily separable space, Asplund space, renormings, biorthogonal system. 



\section{Résumé}

Dans ce travail nous considérons une méthode de constructions génériques d'espaces compacts et clairsemés non métrisables, développée par Baumgartner, Shelah, Rabus, Juhasz et Soukup. Nous introduisons des nouvelles techniques et nous obtenons des nouvelles applications utiles pour l'étude de la topologie des espaces compacts et de la géométrie des espaces de Banach de fonctions continues.

Les nouvelles techniques concernent de nouvelles amalgamations de conditions du forcing qui introduit les espaces clairsemés, ainsi bien que des généralisations des arguments des auteurs cités ci-dessus des points d'un espace compact $K$ aux mesures de Radon sur $K$.

Comme applications, nous obtenons deux nouveaux espaces compacts et clairsemés $K_{1}$ et $K_{2}$, avec les propriétés ci-dessous.

$K_{1}$ est un espace héréditairement séparable de poids $\aleph_{1}$ tel que $C\left(K_{1}\right)$ possède la propriété (C) de Corson et ne possède pas la propriété (E) de Efremov.

$K_{2}$ est le premier exemple d'un espace compact et clairsemé, héréditairement séparable, dont la hauteur est $\omega_{2}$. Il s'ensuit que le degré de Lindelöf héréditaire de $K_{2}$ est $\aleph_{2}$, ce qui montre la consistance de $h L(K) \not \leq h d(K)^{+}$pour les espaces compacts $K . C\left(K_{2}\right)$ est le premier exemple consistant d'un espace de densité $\aleph_{2}$ qui ne possède pas de système biorthogonal non dénombrable.

Mots-clés: espace de Banach $C(K)$, forcing, espace clairsemé, espace héréditairement séparable, espace d'Asplund, renormages, système biorthogonal. 



\section{Sumário}

$\begin{array}{ll}\text { Lista de Símbolos } & 17\end{array}$

$\begin{array}{ll}\text { Introdução } & 19\end{array}$

1 Definições e resultados preliminares $\quad 25$

1.1 Teoria dos conjuntos . . . . . . . . . . . . . . . . 25

1.2 Topologia . . . . . . . . . . . . . . . . . . 26

1.3 Espaços de Banach . . . . . . . . . . . . . . . . . . . 32

1.4 Espaços de Banach de funções contínuas . . . . . . . . . . . . . . . . 35

2 Construindo espaços dispersos genéricos $\quad 37$

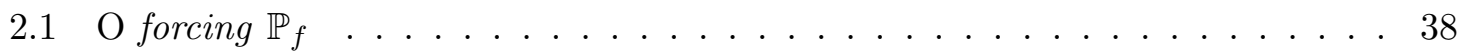

2.2 Propriedades preliminares de $\mathbb{P}_{f} \ldots \ldots \ldots$. . . . . . . . . . . . 39

2.3 Os espaços topológicos genéricos $L$ e $K \ldots \ldots \ldots$. . . . . . . . 41

2.4 Os espaços de Banach $C_{0}(L)$ e $C(K) \ldots \ldots \ldots \ldots \ldots$

2.5 Amalgamações . . . . . . . . . . . . . . . . . . 43

3 Um espaço de Asplund e suas propriedades topológicas $\quad 61$

3.1 Separabilidade hereditária . . . . . . . . . . . . . . . . 62 
3.2 Convergência de seqüências na topologia fraca* . . . . . . . . . . . . 64

3.3 A propriedade $(\mathrm{C})$ e a propriedade $(\mathrm{E}) \ldots \ldots \ldots$. . . . . . . . 68

4 Um espaço de Asplund sem sistema biortogonal não-enumerável $\quad 71$

4.1 Separabilidade hereditária . . . . . . . . . . . . . . . 73

4.2 Conseqüências topológicas e geométricas . . . . . . . . . . . . . . . 75

A Pântanos e a propriedade $\Delta \quad 79$

A.1 Pântanos simplificados . . . . . . . . . . . . . . . 80

A.2 Forcings $\mathcal{F}$-próprios $\ldots \ldots \ldots \ldots \ldots \ldots$

A.3 Construindo a função $f \ldots \ldots \ldots$. . . . . . . . . . . . . . . 85

B Constructions génériques d'espaces d'Asplund C(K) 91

Referências Bibliográficas $\quad 99$

$\begin{array}{ll}\text { Índice Remissivo } & 107\end{array}$ 


\title{
Lista de Símbolos
}

\author{
$\mathbb{N}$ o conjunto dos números naturais \\ $\mathbb{R}$ o corpo dos números reais \\ $\aleph_{0} \quad$ o cardinal enumerável infinito \\ $\aleph_{1} \quad$ o primeiro cardinal não-enumerável \\ $\aleph_{2} \quad$ o segundo cardinal não-enumerável \\ $\omega \quad$ o primeiro ordinal enumerável infinito \\ $\omega_{1} \quad$ o primeiro ordinal não-enumerável \\ $\omega_{2} \quad$ o primeiro ordinal de cardinalidade $\aleph_{2}$ \\ c o cardinal do contínuo \\ $|X|$ a cardinalidade do conjunto $X$ \\ $[X]^{\kappa} \quad$ o conjunto dos subconjuntos de $X$ de cardinalidade $\kappa$ \\ $\|x\| \quad$ a norma do vetor $x$ \\ $X^{*} \quad$ o espaço dual topológico do espaço de Banach $X$ \\ $B_{X}$ a bola unitária fechada do espaço de Banach $X$ \\ $S_{X} \quad$ a esfera unitária do espaço de Banach $X$ \\ [A] o subespaço de $X$ gerado pelo conjunto $A$ \\ $\bar{A}^{w} \quad$ o fecho do conjunto $A$ com respeito à topologia fraca \\ $\bar{A}^{w^{*}}$ o fecho do conjunto $A$ com respeito à topologia fraca* \\ $\chi_{U} \quad$ a função característica do conjunto $U$ \\ $p \Vdash$ a condição $p$ força que
}





\section{Introdução}

Um espaço de Banach $X$ é dito um espaço de Asplund se toda função contínua e convexa definida em $X$ a valores reais é Fréchet-diferenciável em todos os pontos de um subconjunto $G_{\delta}$ denso de $X$.

Em 1975, Asplund [2] mostrou que os espaços de uma certa classe de espaços de Banach, que inclui os espaços $c_{0}(\Gamma)$ (onde $\Gamma$ é um conjunto qualquer) e os espaços reflexivos, possuem essa propriedade. Desde então, apareceram na literatura diversas caracterizações dos espaços de Asplund (veja, por exemplo, [29], [42] e [46]) e eles passaram a ter um papel importante na teoria dos espaços de Banach e, em particular, na teoria de renormações.

Para espaços de Banach separáveis $X$, temos que uma conjunção de resultados devidos a diversos matemáticos, incluindo Asplund, Gregory, Kadec, Klee, Namioka, Phelps e Stegall, entre outros, implica que $X$ admite uma renormação Fréchet-diferenciável se, e somente se, $X$ admite uma função bump Fréchet-diferenciável se, e somente se, $X$ é um espaço de Asplund.

É natural perguntarmos então: o que acontece no caso não-separável?

Para espaços de Banach quaisquer, é fácil construir, a partir de uma norma equivalente Fréchet-diferenciável, uma função bump Fréchet-diferenciável e pode-se mostrar que se $X$ admite uma função bump Fréchet-diferenciável, então $X$ é um espaço de Asplund. Resta, portanto, saber se as recíprocas também são verdadeiras. 
A classe dos espaços de Banach $C(K)$ fornece interessantes exemplos de espaços de Banach não-separáveis (veja, por exemplo, [9], [24], [39], [40], [48] e [52]). Aqui temos mais uma situação em que isso acontece: muitos dos exemplos interessantes de espaços de Asplund não-separáveis são espaços $C(K)$. Namioka e Phelps [46] mostraram que, dado um espaço compacto $K$ (ou localmente compacto $L$ ), temos que $C(K)$ (ou $C_{0}(L)$ ) é de Asplund se, e somente se, $K$ (ou $L$ ) é disperso, isto é, se todo subespaço de $K$ (ou $L$ ) tem pontos isolados.

Os exemplos de espaços compactos e dispersos mais simples de se definir são: a compactificação de Alexandroff de um espaço discreto; e um ordinal sucessor com a topologia da ordem. Mazurkiewicz e Sierpiński [44] mostraram que todo espaço compacto, métrico e disperso é homeomorfo a um ordinal enumerável com a topologia da ordem.

Entretanto, estamos interessados em espaços de Asplund não-separáveis e, portanto, vamos considerar apenas espaços dispersos não-metrizáveis. Existem na literatura diversas construções destes espaços, que não são homeomorfos a ordinais, construídos por métodos conjuntísticos ([17], [28], [49], [56]) e mesmo compactos ou localmente compactos ([3], [36], [38]). O primeiro exemplo de um espaço compacto e disperso de altura $\omega_{2}$ e largura enumerável é o de Baumgartner e Shelah [5], obtido por forcing.

Lembrando que estamos interessados nas propriedades dos espaços de Asplund $C(K)$ não-separáveis, retornemos às perguntas que nos fizemos: será que todo espaço de Asplund admite uma renormação Fréchet-diferenciável ou admite uma função bump Fréchetdiferenciável, tal como acontece com espaços de Asplund separáveis?

Haydon [25] construiu uma árvore $T$ tal que $C_{0}(T)$ não admite renormação Gâteauxdiferenciável. Árvores são espaços localmente compactos e dispersos e temos, portanto, que $C_{0}(T)$ é um espaço de Asplund; como a Fréchet-diferenciabilidade de uma função implica sua Gâteaux-diferenciabilidade, segue que esse espaço $C_{0}(T)$ é um exemplo de um espaço de Asplund que não admite renormação Fréchet-diferenciável.

Mas Haydon [26] provou também que para toda árvore $T, C_{0}(T)$ admite uma função 
bump Fréchet-diferenciável, de forma que o exemplo construído em seu trabalho anterior, assim como qualquer outra árvore, não pode resolver o seguinte problema, ainda sem resposta: existe um espaço de Asplund que não admite uma função bump Fréchet-diferenciável?

$\mathrm{Na}$ esperança de respondermos esta pergunta negativamente através de um espaço da forma $C(K)$, o resultado de Haydon nos obriga a buscar exemplos de espaços compactos (ou localmente compactos) e dispersos, não-metrizáveis que não são árvores.

O espaço $C(K)$, onde $K$ é a reta de Kunen (cuja construção foi reproduzida em [48]), é um dos espaços de funções contínuas com mais aplicações na teoria de espaços de Banach: $K$ é um espaço compacto e disperso, construído sob a hipótese do contínuo, tal que toda potência finita é hereditariamente separável. Pode-se então mostrar que o correspondente espaço de Asplund $C(K)$ não admite renormação Fréchet-diferenciável e não possui um sistema biortogonal não-enumerável (veja [31] e [47]). Entretanto, não se sabe se $C(K)$ admite uma renormação Gâteaux-diferenciável nem se $C(K)$ admite uma função bump Fréchetdiferenciável. Os espaços que vamos estudar neste trabalho possuem propriedades similares.

Nosso objetivo é apresentar um método de construção por forcing de espaços compactos e dispersos não-metrizáveis $K$ e analisar as propriedades topológicas e geométricas dos correspondentes espaços de Asplund $C(K)$. Este método é baseado no espaço construído por Rabus [53] para mostrar a consistência da existência de um espaço inicialmente $\omega_{1}$ compacto $^{1}$ de tightness enumerável e não compacto ${ }^{2}$, respondendo, assim, uma pergunta de Dow e van Douwen. Esta construção, por sua vez, foi inspirada naquela de Baumgartner e Shelah [5]. Em [33], Juhasz e Soukup fazem uma abordagem alternativa da construção de Rabus, usando a linguagem topológica ao invés da linguagem de álgebras de Boole.

O trabalho é composto por quatro capítulos seguidos por dois apêndices: no primeiro capítulo listamos resultados que serão necessários adiante.

\footnotetext{
${ }^{1} \mathrm{Um}$ espaço é inicialmente $\omega_{1}$-compacto se todo recobrimento aberto de cardinalidade menor ou igual a $\aleph_{1}$ possui um subrecobrimento finito.

${ }^{2}$ Cabe notar que, na realidade, Rabus [53] constrói uma álgebra de Boole superatômica, cujo espaço de Stone possui um ponto distinguido, de forma que, ao retirarmos tal ponto, o espaço obtido possui as propriedades que mencionamos.
} 
No segundo capítulo, apresentamos o método de construção de espaços compactos e dispersos a que já nos referimos: definimos um forcing que introduz uma topologia sobre um ordinal não-enumerável $\theta \leq \omega_{2}$ e que depende, assim como em [33], de uma função que associa subconjuntos enumeráveis de $\theta$ a pares de $\theta$. As propriedades de $f$ influenciam as propriedades da topologia introduzida sobre $\theta$.

Após apresentar o forcing e mostrar que ele, de fato, introduz uma topologia sobre $\theta$ de forma que obtemos um espaço compacto e disperso não-metrizável, provamos alguns resultados técnicos sobre o forcing que serão utilizados nos capítulos subseqüentes: os Lemas 2.13 e 2.14. Ambos são resultados combinatórios que garantem a existência de amalgamações, ou seja, de uma condição do forcing que estende outras duas, sob algumas hipóteses a respeito destas. O Lema 2.13 tem hipóteses bastante fortes sobre as condições do forcing a serem estendidas, mas que garantem a possibilidade de se construir uma amalgamação que "copia uma condição dentro da outra". No Lema 2.14, temos uma mistura entre as idéias das amalgamações introduzidas em [33] e [53] e as do Lema 2.13. Aqui, as hipóteses sobre as condições a serem amalgamadas são mais fracas, de forma que a dificuldade para construir uma amalgamação como se quer aumenta.

No terceiro capítulo, fixamos $\theta=\omega_{1}, f=$ min e obtemos um espaço compacto e disperso $K_{1}$ de peso $\aleph_{1}$, de forma que $C\left(K_{1}\right)$ é um espaço de Asplund não-separável. Como a função $f$ aqui é simples e tem propriedades bastante fortes, conseguimos atingir as hipóteses do Lema 2.13 e mostramos, usando esse lema, que toda potência finita de $K_{1}$ é hereditariamente separável. Concluímos que $C\left(K_{1}\right)$ não admite renormação Fréchet-diferenciável e não possui um sistema biortogonal não-enumerável. Passamos daí a analisar outras propriedades de $C\left(K_{1}\right)$ e, generalizando uma idéia de Rabus [53], mostramos que $C\left(K_{1}\right)$ não possui a propriedade (E) de Efremov [18]. Um outro espaço que tem (C) e não tem (E) foi construído por Justin T. Moore (não publicado), usando o princípio conjuntístico $\diamond$, respondendo consistentemente uma pergunta de Plichko e Yost [51].

No quarto capítulo, fixamos $\theta=\omega_{2}$ e uma função $f$ com propriedades mais fortes do que aquela utilizada em [5], [33] e [53]: um resultado não-publicado de Koszmider, 
que apresentamos no Apêndice A, garante a consistência com ZFC da existência dessa função. Assim, obtemos um espaço compacto e disperso $K_{2}$ de peso $\aleph_{2}$, de forma que $C\left(K_{2}\right)$ é, também, um espaço de Asplund não-separável. Aqui, graças às propriedades da função $f$, conseguimos usar o Lema 2.14 para mostrar que toda potência finita de $K_{2}$ é hereditariamente separável.

Mostramos que $K_{2}$ tem altura $\omega_{2}$ e temos, portanto, o primeiro exemplo consistente de um espaço compacto e disperso, hereditariamente separável de altura $\omega_{2}$. Lembramos que a hipótese do contínuo implica que não existe um espaço compacto e disperso de altura $\omega_{2}$ e largura enumerável e Just [35] mostrou, usando o forcing de Cohen, que a não existência destes espaços também é consistente com a negação da hipótese do contínuo. O primeiro exemplo consistente de um espaço compacto e disperso, de largura enumerável e altura $\omega_{2}$ é o de Baumgartner e Shelah [5].

Segue que o grau de Lindelöf hereditário de $K_{2}, h L\left(K_{2}\right)$, é $\aleph_{2}$. Logo, nosso espaço é um contra-exemplo consistente para a desigualdade $h L(K) \leq h d(K)^{+}$, onde $h d(K)$ é a densidade hereditária de $K$. O interesse por essa desigualdade se deve à espécie de dualidade existente entre esses dois cardinais invariantes, juntamente com a seguinte conseqüência de um resultado de Shapirovskiı̌ [62]: para todo espaço compacto $K$, temos que $h d(K) \leq$ $h L(K)^{+}$.

Finalmente, do fato que o peso de $K_{2}$ é $\aleph_{2}$ segue que a densidade de $C\left(K_{2}\right)$ também é $\aleph_{2}$ e como toda potência finita de $K_{2}$ é hereditariamente separável, temos que $C\left(K_{2}\right)$ não admite renormação Fréchet-diferenciável e não possui um sistema biortogonal não-enumerável. Desta forma, este é o primeiro exemplo consistente de um espaço de Banach $C(K)$ de densidade estritamente maior que $\aleph_{1}$ que não possui um sistema biortogonal não-enumerável. Esse exemplo se opõe ao seguinte teorema de Todorcevic [65]: se um espaço de Banach da forma $C(K)$ tem densidade estritamente maior que $\aleph_{1}$, então ele possui um sistema semi-biortogonal não-enumerável. Assim, nosso exemplo mostra que o resultado de Todorcevic não pode ser generalizado em ZFC, substituindo-se a existência de um sistema semibiortogonal não-enumerável pela existência de um sistema biortogonal não-enumerável.

Por fim, apresentamos no Apêndice A a construção de um forcing, usando pântanos 
simplificados, que adiciona a função $f$ que usamos no Capítulo 4, mostrando, assim, que a existência dessa função é consistente com ZFC. Os resultados deste apêndice são devidos a Koszmider e não foram publicados.

O Apêndice B compreende um resumo substancial da presente tese em língua francesa. 


\section{Capítulo 1}

\section{Definições e resultados preliminares}

Nosso objetivo neste capítulo é fixar a notação e lembrar resultados que serão utilizados nos capítulos subseqüentes. Para isso, vamos apresentar as principais definições e enunciar os resultados que usaremos adiante. Procuramos fornecer referências básicas para cada uma das teorias abordadas, onde podem ser encontrados a notação que adotamos e as definições e resultados que eventualmente não incluímos aqui. Indicamos também, sempre que possível, o primeiro trabalho em que cada resultado ou definição apareceu.

\subsection{Teoria dos conjuntos}

Indicamos Jech [30] como referência básica para definições e resultados de teoria dos conjuntos.

Notação 1.1. Dados um ordinal $\alpha$ e um conjunto de ordinais a, dizemos que $\alpha<a$ (resp. $a<\alpha$ ) se para todo $\beta \in a$, tem-se que $\alpha<\beta$ (resp. $\beta<\alpha$ ); se a e b são dois conjuntos de ordinais, dizemos que $a<b$ se para todo $\alpha \in a$, tem-se que $\alpha<b$.

Definição 1.2. Uma família de conjuntos $A$ é um $\Delta$-sistema se existe um conjunto finito $D$ tal que para quaisquer $a_{1}, a_{2} \in A$ distintos, tem-se que $a_{1} \cap a_{2}=D$. O conjunto $D$ é chamado de raiz do $\Delta$-sistema $A$.

O seguinte resultado é conhecido como Lema do $\Delta$-sistema. 
Lema 1.3 (Shanin [61]). Para toda família não-enumerável $A$ de conjuntos finitos, existe uma subfamília não-enumerável $B$ de $A$ que forma um $\Delta$-sistema.

Demonstração. Veja o Teorema 9.18 de [30] ou Teorema 1.5 de [41].

\subsection{Topologia}

Indicamos Engelking [19] como referência básica para definições e resultados de topologia ${ }^{1}$.

Todos os espaços topológicos em questão são de Hausdorff.

Definição 1.4. O peso de um espaço topológico $X, w(X)$, é o menor cardinal infinito $\kappa$ tal que existe uma base topológica de $X$ de cardinalidade menor ou igual a $\kappa$.

Teorema 1.5 (Alexandroff [1]). Todo espaço localmente compacto, não-compacto L possui uma compactificação $K$ tal que $|K \backslash L|=1$ e $w(K)=w(L)$.

Demonstração. Veja o Teorema 3.5.11 de [19].

Notação 1.6. Chamamos o espaço compacto dado pelo teorema acima de compactificação de Alexandroff de $L$ e denotamos $\{*\}=K \backslash L$.

Definição 1.7. Seja $X$ um espaço topológico. Dizemos que $X$ é zero-dimensional se $X$ possui uma base topológica formada por subconjuntos abertos-fechados de $X$.

\section{Densidade e grau de Lindelöf}

Definição 1.8. A densidade de um espaço topológico $X, d(X)$, é o menor cardinal infinito $\kappa$ tal que $X$ possui um subconjunto denso $D$ de cardinalidade menor ou igual a $\kappa$. Se $d(X)=\aleph_{0}$, dizemos que $X$ é separável.

\footnotetext{
${ }^{1}$ Convém mencionar que a maioria das definições e resultados que enunciamos neste capítulo devem-se a diversos matemáticos, incluindo Cantor, Fréchet e Hausdorff, entre outros. Entretanto, na maioria dos casos não indicamos os trabalhos em que essas definições e resultados apareceram pela primeira vez nem seus autores, pois sofreram diversas modificações desde as primeiras versões até chegarem a sua forma atual.
} 
$A$ densidade hereditária de um espaço topológico $X, h d(X)$, é o menor cardinal infinito $\kappa$ tal que para todo subespaço topológico $Y$ de $X$, tem-se que $d(Y) \leq \kappa$. Se $h d(X)=\aleph_{0}$, dizemos que $X$ é hereditariamente separável.

Definição 1.9. Uma família $\left(x_{\alpha}\right)_{\alpha<\kappa}$ de pontos de um espaço topológico $X$ é dita uma seqüência separada à esquerda se existe uma família $\left(V_{\alpha}\right)_{\alpha<\kappa}$ tal que para cada $\alpha<\kappa, V_{\alpha}$ é uma vizinhança aberta de $x_{\alpha}$ e para todo $\alpha<\beta<\kappa$, tem-se que $x_{\alpha} \notin V_{\beta}$.

Proposição 1.10. A densidade hereditária de um espaço topológico é o menor cardinal $\kappa$ tal que X não possui nenhuma seqüência separada à esquerda de cardinalidade $\kappa^{+}$.

Demonstração. Veja o Teorema 3.1 de [56].

Proposição 1.11. Se $K$ é um espaço compacto separável, então $w(K) \leq \mathfrak{c}$.

Demonstração. Veja o Corolário 7.7 de [27].

Definição 1.12. O grau de Lindelöf de um espaço topológico $X, L(X)$, é o menor cardinal infinito $\kappa$ tal que todo recobrimento aberto de $X$ possui um subrecobrimento de cardinalidade menor ou igual a $\kappa$. Se $L(X)=\aleph_{0}$, dizemos que $X$ é Lindelöf.

$O$ grau de Lindelöf hereditário de um espaço topológico $X, h L(X)$, é o menor cardinal infinito $\kappa$ tal que para todo subespaço topológico $Y$ de $X$, tem-se que $L(Y) \leq \kappa$. Se $h L(X)=$ $\aleph_{0}$, dizemos que $X$ é hereditariamente Lindelöf.

Definição 1.13. Uma família $\left(x_{\alpha}\right)_{\alpha<\kappa}$ de pontos de um espaço topológico $X$ é dita uma seqüência separada à direita se existe uma família $\left(V_{\alpha}\right)_{\alpha<\kappa}$ tal que para cada $\alpha<\kappa, V_{\alpha}$ é uma vizinhança aberta de $x_{\alpha}$ e para todo $\alpha<\beta<\kappa$, tem-se que $x_{\beta} \notin V_{\alpha}$.

Proposição 1.14. O grau de Lindelöf hereditário de um espaço topológico é o menor cardinal $\kappa$ tal que $X$ não possui nenhuma seqüência separada à direita de cardinalidade $\kappa^{+}$.

Demonstração. Veja a Proposição 7.6 de [48].

Teorema 1.15 (Shapirovskiü [62]). Para qualquer espaço compacto $K, h d(K) \leq h L(K)^{+}$.

Demonstração. Veja o Teorema 7.17 de [27] 


\section{Medidas de Radon}

Notação 1.16. Dado um espaço topológico $X$, denotamos por Bor $(X)$ a $\sigma$-álgebra dos subconjuntos borelianos de $X$.

Adotamos a seguinte noção de medida de Radon (Definição 18.2.3 de [60]):

Definição 1.17. Seja K um espaço topológico compacto. Uma medida de Radon $\mu$ sobre $K$ é uma medida com sinal, boreliana, limitada e regular, ou seja, é uma função $\mu: \operatorname{Bor}(K) \rightarrow \mathbb{R}$ tal que

- $\mu(\emptyset)=0$;

- para toda familia $\left(B_{n}\right)_{n \in \mathbb{N}}$ de subconjuntos borelianos de $K$ dois a dois disjuntos, temse que

$$
\mu\left(\bigcup_{n \in \mathbb{N}} B_{n}\right)=\sum_{n \in \mathbb{N}} \mu\left(B_{n}\right)
$$

- para todo $B \in \operatorname{Bor}(K)$, tem-se que

$$
|\mu|(B)=\sup \left\{\sum_{i=1}^{k}\left|\mu\left(B_{i}\right)\right|: B=\bigcup_{i=1}^{k} B_{i}, B_{i} \in \operatorname{Bor}(K) \text { e } B_{i} \cap B_{j}=\emptyset, \text { se } i \neq j\right\}<\infty
$$

- para todo $B \in \operatorname{Bor}(K)$ e todo $\varepsilon>0$ existem conjuntos $F$ compacto e $G$ aberto tais que $F \subseteq B \subseteq G$ e $|\mu|(G \backslash F)<\varepsilon$.

Notação 1.18. Dado um espaço topológico compacto $K$, denotamos por $M(K)$ o conjunto das medidas de Radon sobre $K$; para cada $\mu \in M(K)$, denotamos por $\|\mu\|=|\mu|(K)$.

Notação 1.19. Dado um espaço topológico compacto $K$ e $x \in K$, denotamos por $\delta_{x}$ a medida de Dirac com suporte no ponto $x$. Observe que $\delta_{x}$ é uma medida de Radon e $\left\|\delta_{x}\right\|=1$.

\section{Espaços dispersos}

Definição 1.20. Um espaço topológico $X$ é disperso se todo subespaço de $X$ tem um ponto isolado. 
Os exemplos mais comuns de espaços compactos dispersos são: a compactificação de Alexandroff de um espaço discreto e um ordinal sucessor com a topologia da ordem. Mazurkiewicz e Sierpiński [44] mostraram que todo espaço compacto, métrico e disperso é homeomorfo a um ordinal enumerável com a topologia da ordem. Alguns exemplos de espaços dispersos não-metrizáveis interessantes podem ser encontrados em [5] e [49].

Lembramos que um espaço compacto $K$ é disperso se, e somente se, não existe nenhuma função contínua $f: K \rightarrow[0,1]$ sobrejetora se, e somente se, $K$ é zero-dimensional e não existe nenhuma função contínua $f: K \rightarrow 2^{\omega}$ sobrejetora (veja o Teorema 8.5.4 de [60]). Além disso, um espaço compacto zero-dimensional $K$ é disperso se, e somente se, a álgebra de Boole $C l o p(K)$, formada pelos subconjuntos abertos-fechados de $K$, é superatômica (veja o Teorema 1.1 de [57]).

Definição 1.21. Dado um espaço topológico $X$, o conjunto derivado de $X$, que denotamos por $X^{\prime}$, é o conjunto dos pontos de acumulação de $X$, ou seja, o conjunto dos pontos de $X$ que não são isolados em $X$. Definimos recursivamente, para cada ordinal $\alpha, X^{(\alpha+1)}=$ $\left(X^{(\alpha)}\right)^{\prime}$ e para cada ordinal limite $\lambda, X^{(\lambda)}=\bigcap_{\alpha<\lambda} X^{(\alpha)}$.

Teorema 1.22 (Cantor, Bendixon). Seja X um espaço topológico. Então existe um ordinal $\alpha$ tal que $X^{(\alpha)}=X^{(\alpha+1)} . X$ é disperso se, e somente se, existe um ordinal $\alpha$ tal que $X^{(\alpha)}=\emptyset$.

Demonstração. Veja o Teorema 8.5.2 de [60].

Seja $K$ um espaço compacto e disperso e seja $\alpha$ o menor ordinal tal que $K^{(\alpha)}=K^{(\alpha+1)}$. Observe que, como $K$ é disperso, temos que $K^{(\alpha)}=\emptyset$. Daí, do fato que $K$ é compacto segue que $\alpha$ é um ordinal sucessor: senão, $\left(K^{(\beta)}\right)_{\beta<\alpha}$ seria uma família decrescente infinita de subconjuntos fechados e não-vazios de $K$ cuja intersecção é vazia e, pela minimalidade de $\alpha$, não teria nenhuma subfamília finita cuja intersecção fosse vazia, contradizendo a compacidade de $K$. Portanto, $\alpha=\beta+1$, para algum ordinal $\beta$ e observe que $K^{(\beta)}$ é finito.

Definição 1.23. Seja $K$ um espaço compacto e disperso. A altura ${ }^{2}$ de $K$, ht $(K)$, é o menor ordinal $\alpha$ tal que $K^{(\alpha)}$ é finito e a largura de $K, w d(K)$, é o menor cardinal infinito $\kappa$ tal que para todo $\beta<h t(K)$, tem-se que $\left|K^{(\beta)} \backslash K^{(\beta+1)}\right| \leq \kappa$.

\footnotetext{
${ }^{2}$ Enfatizamos que estamos adotando a definição de altura de [5], freqüentemente utilizada para espaços dispersos. Alternativamente, poderia-se definir a altura como o menor ordinal $\alpha$ tal que $K^{(\alpha)}=\emptyset$.
} 
Proposição 1.24. Seja $\theta$ um ordinal e para cada $\xi \in \theta$ seja $V_{\xi} \subseteq \theta$ tal que $\xi=\max V_{\xi}$. Considere $\tau$ a topologia sobre $\theta$ que possui o conjunto

$$
\left\{V_{\xi}: \xi \in \theta\right\} \cup\left\{\theta \backslash V_{\xi}: \xi \in \theta\right\}
$$

como sub-base. Suponha que existe uma função $i:[\theta]^{2} \rightarrow[\theta]^{<\aleph_{0}}$ tal que para quaisquer $\xi<\eta<\theta, i(\{\xi, \eta\}) \subseteq \xi e:$

- se $\xi \in V_{\eta}$, então $V_{\xi} \backslash V_{\eta} \subseteq \bigcup_{\zeta \in i(\{\xi, \eta\})} V_{\zeta}$;

- se $\xi \notin V_{\eta}$, entãa $V_{\xi} \cap V_{\eta} \subseteq \bigcup_{\zeta \in i(\{\xi, \eta\})} V_{\zeta}$.

Então para todo $\xi \in \theta, V_{\xi}$ é compacto $e$

$$
\left\{V_{\xi} \backslash \bigcup_{\eta \in F} V_{\eta}: F \in[\eta]^{<\aleph_{0}}\right\}
$$

forma um sistema fundamental de vizinhanças abertas de $\xi$.

Portanto, $(\theta, \tau)$ é um espaço disperso, localmente compacto e zero-dimensional.

Demonstração. O fato que cada $V_{\xi}$ é compacto segue do Teorema 1.5 de [33].

Seja $U \in \tau$ e $\xi \in U$. Como o conjunto

$$
\left\{V_{\xi}: \xi \in \theta\right\} \cup\left\{\theta \backslash V_{\xi}: \xi \in \theta\right\}
$$

é uma sub-base, existem $F, G \subseteq \theta$ finitos tais que

$$
\xi \in \bigcap_{\eta \in F} V_{\eta} \cap \bigcap_{\eta \in G}\left(\theta \backslash V_{\eta}\right) \subseteq U
$$

Segue do fato que para todo $\eta \in \theta, \max V_{\eta}=\eta$ que para todo $\eta \in F, \eta \geq \xi$. Considere $G_{1}=\{\eta \in G: \xi<\eta\}$ e observe que

$$
\xi \in V_{\xi} \backslash \bigcup\left\{V_{\zeta}: \zeta \in \bigcup_{\eta \in F \cup G_{1}} i(\{\xi, \eta\}) \text { ou } \zeta \in G \backslash G_{1}\right\} \subseteq \bigcap_{\eta \in F} V_{\eta} \cap \bigcap_{\eta \in G}\left(\theta \backslash V_{\eta}\right) \subseteq U
$$


Temos assim que $(\theta, \tau)$ é localmente compacto e zero-dimensional e observe que para todo $X \subseteq \theta, \min X$ é um ponto isolado de $X$, de forma que $(\theta, \tau)$ é disperso.

Proposição 1.25. Para todo espaço compacto e disperso $K$ existe um ordinal $\theta$ e uma topologia $\tau$ sobre $\theta$ tal que $K$ é homeomorfo ao espaço topológico $(\theta, \tau)$ e para cada $\xi \in \theta$, existe uma vizinhança aberta $V_{\xi}$ de $\xi$ tal que $\max V_{\xi}=\xi$ e o conjunto

$$
\left\{V_{\xi}: \xi \in \theta\right\} \cup\left\{\theta \backslash V_{\xi}: \xi \in \theta\right\}
$$

é uma sub-base.

Demonstração. Seja $\alpha$ tal que $K^{(\alpha)}=\emptyset$ e para cada $x \in K$, defina $\operatorname{rank}(x)=\beta<\alpha$, onde $\beta$ é o ordinal tal que $x \in K^{(\beta)} \backslash K^{(\beta+1)}$ e sejam $\theta$ um ordinal e $h: K \rightarrow \theta$ uma bijeção tal que para todo $x, y \in K$, se $\operatorname{rank}(x)<\operatorname{rank}(y)$, então $h(x)<h(y)$. Para cada $x \in K$, seja $U_{x}$ uma vizinhança aberta-fechada de $x$ em $K$ tal que $U_{x} \cap K^{(\operatorname{rank}(x))}=\{x\}$ e para cada $\xi \in \theta$, tome $V_{\xi}=h\left[U_{h^{-1}(\xi)}\right]$. Seja $\tau$ a topologia sobre $\theta$ que possui o conjunto

$$
\left\{V_{\xi}: \xi \in \theta\right\} \cup\left\{\theta \backslash V_{\xi}: \xi \in \theta\right\}
$$

como sub-base. $h$ é uma função bijetora e contínua entre compactos e, portanto, é um homeomorfismo de $K$ sobre $\theta$. Observe que para todo $\xi \in \theta$, temos que $\max V_{\xi}=\xi$.

Definição 1.26. Seja $K$ um espaço compacto ou localmente compacto e $\mu$ uma medida de Radon sobre $K$. $\mu$ é uma medida atômica se existem uma seqüência de reais $\left(a_{n}\right)_{n \in \mathbb{N}}$ e uma seqüência $\left(x_{n}\right)_{n \in \mathbb{N}}$ em $K$ tais que

$$
\mu=\sum_{n \in \mathbb{N}} a_{n} \delta_{x_{n}} \quad \text { e }|\mu|=\sum_{n \in \mathbb{N}}\left|a_{n}\right|<\infty .
$$

Teorema 1.27. Seja $K$ um espaço compacto ou localmente compacto. $K$ é disperso se, e somente se, toda medida de Radon sobre $K$ é atômica.

Demonstração. Veja o Teorema 19.7.6 de [60]. 


\subsection{Espaços de Banach}

Indicamos Fabian et al [20] como referência básica para a teoria geral de espaços de Banach e Deville, Godefroy e Zizler [15] para a teoria de renormações ${ }^{3}$ e espaços de Asplund.

Todos os espaços de Banach em questão são espaços de Banach reais de dimensão infinita.

Teorema 1.28. Todo subconjunto convexo e fechado de um espaço de Banach é fracamente fechado.

Demonstração. Veja o Teorema 3.19 de [20].

\section{Espaços de Asplund}

Definição 1.29. Uma função $f$ definida num espaço de Banach $X$ a valores reais é dita Fréchet-diferenciável em $x \in X$ se existe $f^{\prime}(x) \in X^{*}$ tal que $\lim _{y \rightarrow 0} \frac{f(x+y)-f(x)-f^{\prime}(x) y}{\|y\|}=0$.

Dizemos que uma norma $\|\cdot\|$ em um espaço de Banach $X$ é Fréchet-diferenciável se $\|\cdot\|$ é Fréchet-diferenciável em todo $x \in S_{X}$.

Definição 1.30 (Asplund [2]). Um espaço de Banach $X$ é um espaço de Asplund se toda função contínua e convexa $f: X \rightarrow \mathbb{R}$ é Fréchet-diferenciável em todos os pontos de um subconjunto $G_{\delta}$ denso de $X$.

O seguinte resultado, devido a diversos matemáticos, incluindo Asplund, Gregory, Namioka e Stegall, fornece uma bela caracterização desses espaços:

Teorema 1.31. Um espaço de Banach é de Asplund se, e somente se, o dual de todo subespaço separável é separável.

Demonstração. Veja o Teorema 5.7 de [15] ou o Teorema 8.26 de [20].

Definição 1.32. Dado um espaço de Banach $X$, dizemos que uma função $f: X \rightarrow \mathbb{R}$ é uma função bump se ela tem suporte limitado e não-vazio.

\footnotetext{
${ }^{3}$ Renormar um espaço de Banach é substituir a norma dada por uma norma equivalente.
} 
O seguinte teorema se deve a uma conjunção de resultados devidos a diversos matemáticos (veja [21]):

Teorema 1.33. Dado um espaço de Banach $X$, temos que $(a) \Rightarrow(b) \Rightarrow(c)$, onde:

(a) X admite uma renormação Fréchet-diferenciável;

(b) X admite uma função bump Fréchet-diferenciável;

(c) X é um espaço de Asplund.

Além disso, se $X$ é separável, então $(c) \Rightarrow(a)$ e, portanto, $(a),(b)$ e (c) são equivalentes.

Demonstração. Veja o Teorema 5.3 de [15].

\section{A estrutura dos espaços de Banach}

Definição 1.34 (Mazur [43]). A norma de um espaço de Banach X possui a propriedade de interseç̧ão de Mazur se todo subconjunto convexo, fechado e limitado de $X$ se escreve como uma intersecção de bolas fechadas.

Teorema 1.35 (Mazur [43]). Se a norma de um espaço de Banach X é Fréchet-diferenciável, então ela tem a propriedade de intersecção de Mazur.

Demonstração. Veja a Proposição 4.5 de [15].

Proposição 1.36 (Jiménez-Sevilla, Moreno [31]). Se X é um espaço de Banach cuja norma tem a propriedade de intersecção de Mazur, então existe um subconjunto limitado $A$ de $X$ tal que $|A|=d(X)$ e para todo $x_{0} \in A$, tem-se que $\left.x_{0} \notin \overline{\left[A \backslash\left\{x_{0}\right\}\right.}\right]^{w}$.

Demonstração. Segue da Proposição 4.1 de [31].

Definição 1.37. Um sistema semi-biortogonal de um espaço de Banach $X$ é uma família de pares $\left(x_{\alpha}, \varphi_{\alpha}\right)_{\alpha<\kappa} \subseteq X \times X^{*}$ tal que para todo $\alpha, \beta<\kappa$, tem-se que:

- se $\alpha<\beta$, então $\varphi_{\beta}\left(x_{\alpha}\right)=0$; 
- se $\alpha=\beta$, então $\varphi_{\beta}\left(x_{\alpha}\right)=1$;

- se $\alpha>\beta$, então $\varphi_{\beta}\left(x_{\alpha}\right) \geq 0$.

Um sistema biortogonal de um espaço de Banach $X$ é uma família de pares $\left(x_{\alpha}, \varphi_{\alpha}\right)_{\alpha<\kappa} \subseteq$ $X \times X^{*}$ tal que para todo $\alpha, \beta \in \kappa$, tem-se que:

- se $\alpha \neq \beta$, então $\varphi_{\beta}\left(x_{\alpha}\right)=0$;

- se $\alpha=\beta$, então $\varphi_{\beta}\left(x_{\alpha}\right)=1$.

Observe que todo sistema biortogonal é um sistema semi-biortogonal.

Teorema 1.38. Seja X um espaço de Banach não-separável e hereditariamente Lindelöf com respeito à topologia fraca. Então X não admite renormação Fréchet-diferenciável e não possui nenhum sistema biortogonal não-enumerável.

Demonstração. Suponhamos que $X$ é não-separável e admite uma renormação Fréchetdiferenciável e mostremos que $X$ não é hereditariamente Lindelöf com respeito à topologia fraca. Segue do Teorema 1.35, que $X$ admite uma renormação com a propriedade de intersecção de Mazur. Pelo Teorema 1.36, existe uma família limitada $\left(x_{\alpha}\right)_{\alpha<\omega_{1}} \subseteq X$ tal que para todo $\alpha<\omega_{1}$,

$$
x_{\alpha} \notin{\overline{\left[x_{\beta}: \beta<\omega_{1} \text { e } \beta \neq \alpha\right]}}^{w} \text {. }
$$

Logo, $\left\{x_{\alpha}: \alpha<\omega_{1}\right\}$ é uma seqüência separada à direita com respeito à topologia fraca. Pela Proposição 1.14, temos que $X$ não é hereditariamente Lindelöf com respeito à topologia fraca.

Suponhamos agora que $\left(x_{\alpha}, \varphi_{\alpha}\right)_{\alpha<\omega_{1}} \subseteq X \times X^{*}$ é um sistema biortogonal não-enumerável de $X$. Temos que para todo $\alpha<\omega_{1}$,

$$
x_{\alpha} \notin{\overline{\left[x_{\beta}: \beta<\omega_{1} \text { e } \beta \neq \alpha\right]}}^{w}
$$

Logo, $\left\{x_{\alpha}: \alpha<\omega_{1}\right\}$ é uma seqüência separada à direita em $X$, com respeito à topologia fraca. Pela Proposição 1.14, temos que $X$ não é hereditariamente Lindelöf com respeito à topologia fraca. 


\subsection{Espaços de Banach de funções contínuas}

Indicamos Fabian et al [20] e Semadeni [60] como referências básicas para a teoria dos espaços de Banach $C(K)$.

Notação 1.39. Seja $K$ um espaço compacto (resp. L um espaço localmente compacto). Denotamos por $C(K)$ (resp. $C_{0}(L)$ ) o espaço de Banach das funções contínuas definidas em $K$ a valores reais (resp. o espaço das funções contínuas definidas em $L$ a valores reais que se anulam no infinito), munido da norma do supremo.

Começamos recordando a seguinte versão do Teorema de Stone-Weierstrass:

Teorema 1.40 (Stone [64]; Weierstrass [68]). Seja K um espaço compacto (resp. L localmente compacto) e zero-dimensional e seja Clop $(K)$ (resp. Clop $(L))$ a álgebra dos subconjuntos abertos-fechados de $K$ (resp. L). Então o conjunto

$$
\left\{\sum_{i=1}^{n} a_{i} \chi_{\mathcal{U}_{i}}: n \in \mathbb{N} \text { e } \forall 1 \leq i \leq n, \mathcal{U}_{i} \in C l o p(K)(\operatorname{resp} . \operatorname{Clop}(L)), a_{i} \in \mathbb{Q}\right\}
$$

é denso em $C(K)$ (resp. $C_{0}(L)$ ), com respeito à topologia da norma.

Demonstração. Veja o Teorema 7.3 .8 de [60].

Segue o seguinte:

Teorema 1.41. Seja K um espaço compacto (resp. L localmente compacto) zero-dimensional. A densidade de $C(K)$ (resp. $\left.C_{0}(L)\right)$ é igual ao peso de $K$ (resp. L).

Demonstração. Segue facilmente do teorema anterior.

Teorema 1.42 (Riesz [55]). Seja K é um espaço compacto. Então a aplicação $T: M(K) \rightarrow$ $C(K)^{*}$ dada por

$$
T(\mu)(f)=\int_{K} f d \mu
$$

é um isomorfismo isométrico. 
Demonstração. Veja o Teorema 8.4.1 de [60].

Teorema 1.43 (Namioka, Phelps [46]). Seja K um espaço compacto (resp. L localmente compacto). $K$ (resp. L) é disperso se, e somente se, $C(K)$ (resp. $\left.C_{0}(L)\right)$ é um espaço de Asplund.

Demonstração. Veja o Lema 8.6 de [15].

Teorema 1.44. Se $K$ é um espaço compacto e disperso tal que para todo $n \in \mathbb{N}, K^{n}$ é hereditariamente separável, então $C(K)$ é hereditariamente Lindelöf com respeito à topologia fraca.

Demonstração. Segue do Lema 7.2 e dos Teoremas 7.3 e 7.4 de [48].

Corolário 1.45. Seja $K$ um espaço compacto, disperso e zero-dimensional tal que para todo $n \in \mathbb{N}, K^{n}$ é hereditariamente separável. Se $w(K)>\aleph_{0}$, então $C(K)$ não admite renormação Fréchet-diferenciável e não possui nenhum sistema biortogonal não-enumerável.

Demonstração. Pelo Teorema 1.44, temos que $C(K)$ é hereditariamente Lindelöf com respeito à topologia fraca. Por outro lado, segue do Teorema 1.41 que $d(C(K))=w(K)>$ $\aleph_{0}$, ou seja, $C(K)$ é um espaço de Banach não-separável e hereditariamente Lindelöf com respeito à topologia fraca. Logo, pelo Teorema 1.38, temos que $C(K)$ não admite renormação Fréchet-diferenciável e não possui nenhum sistema biortogonal não-enumerável.

Teorema 1.46 (Todorcevic [65]). Seja $K$ um espaço compacto. Se $d(C(K))>\aleph_{1}$, então $C(K)$ possui sistemas semi-biortogonais não-enumeráveis.

Demonstração. Veja o Teorema 9 de [65]. 


\section{Capítulo 2}

\section{Construindo espaços dispersos genéricos}

Neste capítulo, vamos apresentar um método de construção de espaços (localmente) compactos e dispersos usando forcing. Ostaszewski [49] construiu, usando o princípio conjuntístico $\diamond$, o primeiro exemplo de um espaço localmente compacto e disperso, de largura enumerável e altura $\omega_{1}$, enumeravelmente compacto e perfeitamente normal. Há, na literatura, diversas construções de espaços similares (veja, por exemplo, [32] e [54]), todas elas respondendo a seguinte pergunta de Telgársky: existe um espaço localmente compacto e disperso, de largura enumerável e altura $\omega_{1}$ ? Juhasz e Weiss [34] generalizaram os resultados acima citados, mostrando que para cada ordinal $\alpha<\omega_{2}$, existe um espaço localmente compacto e disperso de largura enumerável e altura $\alpha$.

A pergunta natural que segue é se existem espaços compactos e dispersos, de largura enumerável e altura $\omega_{2}$. Segue da Proposição 1.11 que a hipótese do contínuo implica que não; Just [35] mostrou que no modelo de Cohen, no qual vale a negação da hipótese do contínuo, também não existem tais espaços. Por outro lado, Baumgartner e Shelah [5] provaram, por forcing, que a existência de um espaço desta forma é consistente com ZFC.

Rabus [53] modificou o forcing utilizado em [5] para obter um espaço de tightness enumerável, inicialmente $\omega_{1}$-compacto e não-compacto, respondendo uma pergunta de Dow e van Douwen: seu espaço é obtido retirando-se um ponto distinguido do espaço de Stone de 
uma álgebra de Boole introduzida por forcing. Em [33], Juhasz e Soukup apresentam uma construção alternativa à de Rabus e generalizam os resultados deste, usando a linguagem topológica ao invés da algébrica.

Nosso objetivo neste capítulo é apresentar uma versão geral do forcing utilizado em [33] e mostrar algumas propriedades do espaço topológico que esse forcing adiciona. Começamos pela definição do forcing, que depende de um ordinal não-enumerável $\theta \leq \omega_{2}$ e de uma função $f$ que associa subconjuntos enumeráveis de $\theta$ a pares de $\theta$ e mostramos algumas propriedades básicas do forcing que dependem de $f$. Na Seção 2.3, definimos a topologia que o forcing introduz sobre $\theta$ e verificamos que o espaço topológico $L$ obtido é localmente compacto. Na seção seguinte, concluímos que $C(K)$ é um espaço de Asplund não-separável, onde $K$ é a compactificação de Alexandroff de $L$.

Por fim, na Seção 2.5, mostramos dois lemas técnicos que serão chave nos capítulos subseqüentes. Em cada um deles, assumindo uma certa uniformidade entre duas condições do forcing, construímos uma nova amalgamação que garantirá que os espaços dos Capítulos 3 e 4 sejam hereditariamente separáveis.

\section{$2.1 \quad$ O forcing $\mathbb{P}_{f}$}

Definição 2.1. Dados um ordinal não-enumerável $\theta \leq \omega_{2}$ e uma função $f:[\theta]^{2} \rightarrow[\theta] \leq \aleph_{0}$ tal que para quaisquer $\xi, \eta \in \theta, \xi \neq \eta, f(\{\xi, \eta\}) \subseteq \min \{\xi, \eta\}$, seja $\mathbb{P}_{f}$ o forcing formado pelas condições $p=\left(D_{p}, h_{p}, i_{p}\right)$ onde:

1. $D_{p} \in[\theta]^{<\aleph_{0}}$

2. $h_{p}: D_{p} \rightarrow \wp\left(D_{p}\right)$ e para todo $\xi \in D_{p}$, tem-se que $\max h_{p}(\xi)=\xi$;

3. $i_{p}:\left[D_{p}\right]^{2} \rightarrow\left[D_{p}\right]^{<\aleph_{0}}$ e para todo $\xi, \eta \in D_{p}, \xi<\eta$, tem-se que:

(a) se $\xi \in h_{p}(\eta)$, então $h_{p}(\xi) \backslash h_{p}(\eta) \subseteq \bigcup_{\gamma \in i_{p}(\{\xi, \eta\})} h_{p}(\gamma)$,

(b) se $\xi \notin h_{p}(\eta)$, então $h_{p}(\xi) \cap h_{p}(\eta) \subseteq \bigcup_{\gamma \in i_{p}(\{\xi, \eta\})} h_{p}(\gamma)$,

(c) $e i_{p}(\{\xi, \eta\}) \subseteq f(\{\xi, \eta\})$;

ordenado por $p \leq q$ se $D_{p} \supseteq D_{q}$, para todo $\xi \in D_{q}, h_{p}(\xi) \cap D_{q}=h_{q}(\xi)$ e $\left.i_{p}\right|_{\left[D_{q}\right]^{2}}=i_{q}$. 
Notação 2.2. Dado $p=\left(D_{p}, h_{p}, i_{p}\right) \in \mathbb{P}_{f}$, chamamos $D_{p}$ de domínio da condição $p$ e para cada $\xi, \eta \in D_{p}, \xi<\eta$, denotamos

$$
h_{p}(\xi) * h_{p}(\eta)= \begin{cases}h_{p}(\xi) \backslash h_{p}(\eta) & \text { se } \xi \in h_{p}(\eta), \\ h_{p}(\xi) \cap h_{p}(\eta) & \text { se } \xi \notin h_{p}(\eta) .\end{cases}
$$

Observemos que com esta notação podemos reescrever as condições 3.(a) e (b) da Definição 2.1 da seguinte maneira: para todo $\xi, \eta \in D_{p}, \xi<\eta$, tem-se que

$$
h_{p}(\xi) * h_{p}(\eta) \subseteq \bigcup_{\gamma \in i_{p}(\{\xi, \eta\})} h_{p}(\gamma)
$$

Note que a operação $*$ não é comutativa e também que $\xi \notin h_{p}(\xi) * h_{p}(\eta)$ de forma que $h_{p}(\xi) * h_{p}(\eta) \subseteq \xi$

Tendo em vista a Proposição 1.25, se quisermos forçar uma topologia sobre o ordinal $\theta$ de forma a obter um espaço disperso, as condições 1 e 2 da Definição 2.1 acima são exigências naturais: para cada $\xi \in D_{p}$, vamos determinar, dentre os elementos de $D_{p}$, aqueles que estão na vizinhança $V_{\xi}$ da caracterização, ou seja, $h_{p}(\xi)$ é uma aproximação finita para $V_{\xi}$.

Queremos também que a topologia introduzida sobre $\theta$ faça do espaço obtido um espaço localmente compacto, ou seja, queremos que cada vizinhança $V_{\xi}$ seja compacta. Pela Proposição 1.24, precisamos daquela função $i$ e as condições 3.(a) e (b) da Definição 2.1 vão garantir que $i_{p}$ é uma aproximação finita para $i$.

Mas para que serve, afinal, a condição 3.(c) da Definição 2.1? Pois bem: se tomamos a definição sem essa última condição, o forcing não tem c.c.c. e não preserva cardinais. Isso prejudicaria todo o projeto: por exemplo, não teríamos como garantir, neste caso, que $\theta$ é um ordinal não-enumerável no modelo estendido, ou seja, não teríamos que o espaço obtido é um espaço não-metrizável.

\subsection{Propriedades preliminares de $\mathbb{P}_{f}$}

Nesta capítulo, $\theta$ é um ordinal não-enumerável menor ou igual a $\omega_{2}$ e $f:[\theta]^{2} \rightarrow[\theta] \leq \aleph_{0}$ é uma função tal que para quaisquer $\xi, \eta \in \theta, \xi \neq \eta, f(\{\xi, \eta\}) \subseteq \min \{\xi, \eta\}$. 
Lema 2.3 (Juhasz, Soukup [33]). Para cada $\xi<\theta$, o conjunto $\left\{p \in \mathbb{P}_{f}: \xi \in D_{p}\right\}$ é denso em $\mathbb{P}_{f}$.

Demonstração. Veja o Lema 2.2 de [33].

Lembremos a definição da propriedade $\Delta$ :

Definição 2.4 (Baumgartner, Shelah [5]). Dado um ordinal não-enumerável $\theta \leq \omega_{2}$, dizemos que uma função $f:[\theta]^{2} \rightarrow[\theta] \leq \aleph_{0}$ tem a propriedade $\Delta$ se para todo $\xi<\eta<\theta$, temos que $f(\{\xi, \eta\}) \subseteq \xi$ e se para toda família não-enumerável $A$ de subconjuntos finitos de $\theta$, existem $a, b \in A$ distintos tais que para todo $\zeta \in a \cap b$, todo $\xi \in a \backslash b$ e todo $\eta \in b \backslash a$ :

(i) se $\zeta<\xi, \eta$, então $\zeta \in f(\{\xi, \eta\})$;

(ii) se $\zeta<\xi$, então $f(\{\zeta, \eta\}) \subseteq f(\{\xi, \eta\})$;

(iii) se $\zeta<\eta$, então $f(\{\zeta, \xi\}) \subseteq f(\{\xi, \eta\})$.

Teorema 2.5 (Rabus [53]; Juhasz, Soukup [33]). Se $f$ tem a propriedade $\Delta$, então $\mathbb{P}_{f}$ tem c.c.c.

Demonstração. Veja o Lema 4.1 de [53] ou o Teorema 2.4 de [33].

Segue daí (Teorema 5.10, Cap.VII, [41]) que se $f$ tem a propriedade $\Delta$, então o forcing $\mathbb{P}_{f}$ preserva cardinais.

Dado um subconjunto $X$ de $\theta$ e uma condição $p \in \mathbb{P}_{f}$, definimos $\left.p\right|_{X}=\left(D_{\left.p\right|_{X}}, h_{\left.p\right|_{X}}, i_{\left.p\right|_{X}}\right)$ da seguinte forma: $D_{\left.p\right|_{X}}=D_{p} \cap X$; para cada $\xi \in D_{\left.p\right|_{X}}, h_{\left.p\right|_{X}}(\xi)=h_{p}(\xi) \cap X$; e para cada $\operatorname{par}\{\xi, \eta\} \subseteq D_{p \mid X}, i_{\left.p\right|_{X}}(\{\xi, \eta\})=i_{p}(\{\xi, \eta\}) \cap X$. Convém notar que se $p \in \mathbb{P}_{f}$ e $X \subseteq \theta$, nem sempre vale que $\left.p\right|_{X} \in \mathbb{P}_{f}$. Mas temos o seguinte:

Lema 2.6 (Rabus [53]). Sejam $p_{1}, p_{2} \in \mathbb{P}_{f}$ e $B \subseteq \theta$ tais que $D_{p_{1}} \subseteq B, B<D_{p_{2}} \backslash B$ e se $\xi \in B$ e $\eta \in B \cup D_{p_{2}}$, então $f(\{\xi, \eta\}) \subseteq B$. Se $q \in \mathbb{P}_{f}$ é tal que $q \leq p_{1}, p_{2}$, então $\left.q\right|_{B \cup D_{p_{2}}} \in \mathbb{P}_{f}$ e $\left.q\right|_{B \cup D_{p_{2}}} \leq p_{1}, p_{2}$. 
Demonstração. Suponhamos $p_{1}=\left(D_{1}, h_{1}, i_{1}\right), p_{2}=\left(D_{2}, h_{2}, i_{2}\right) \in \mathbb{P}_{f}$ e $B \subseteq \theta$ como na hipótese. Seja $q \in \mathbb{P}_{f}, q \leq p_{1}$, $p_{2}$ e vejamos que $\left.q\right|_{B \cup D_{2}} \in \mathbb{P}_{f}$. É fácil ver que $\left.q\right|_{B \cup D_{2}}$ satisfaz as condições 1,2 e 3.(c) da Definição 2.1 .

Para verificar que $\left.q\right|_{B \cup D_{2}}$ satisfaz as condições 3.(a) e (b) da Definição 2.1, fixemos $\xi, \eta \in D_{q} \cap\left(B \cup D_{2}\right), \xi<\eta$.

Notemos que se $\xi \in B$ e $\eta \in B \cup D_{2}$, então $i_{q}(\{\xi, \eta\}) \subseteq B$ e, portanto, $i_{\left.q\right|_{B \cup D_{2}}}(\{\xi, \eta\})=$ $i_{q}(\{\xi, \eta\})$. Se $\xi \in D_{2} \backslash B$, então $\eta \in D_{2}$ e $i_{q}(\{\xi, \eta\}) \subseteq D_{2}$ e, portanto, $i_{\left.q\right|_{B \cup D_{2}}}(\{\xi, \eta\})=$ $i_{q}(\{\xi, \eta\})$.

Tomemos $\zeta \in\left(h_{q}(\xi) * h_{q}(\eta)\right) \cap\left(B \cup D_{2}\right)$.

Caso 1. $\xi \in B$.

Neste caso, temos, por hipótese, que $f(\{\xi, \eta\}) \subseteq B$. Como $q \in \mathbb{P}_{f}$, existe $\delta \in i_{q}(\{\xi, \eta\}) \subseteq$ $f(\{\xi, \eta\}) \subseteq B$ tal que $\zeta \in h_{q}(\delta)$. Logo, $\delta \in i_{q}(\{\xi, \eta\}) \cap B \subseteq i_{\left.q\right|_{B \cup D_{2}}}(\{\xi, \eta\})$ e como $\zeta \leq \xi$, $\zeta \in B \cup D_{2}$ e $\xi \in B$, segue que $\zeta \in B$ e, portanto, $\zeta \in h_{q}(\delta) \cap B \subseteq h_{\left.q\right|_{B \cup D_{2}}}(\delta)$.

Caso 2. $\xi \in D_{2} \backslash B$.

Neste caso, temos que $i_{q}(\{\xi, \eta\})=i_{2}(\{\xi, \eta\}) \subseteq D_{2}$. Logo, $i_{\left.q\right|_{B \cup D_{2}}}(\{\xi, \eta\})=i_{q}(\{\xi, \eta\})$. Como existe $\delta \in i_{q}(\{\xi, \eta\})$ tal que $\zeta \in h_{q}(\delta)$ e $\zeta \in B \cup D_{2}$, temos que $\zeta \in h_{\left.q\right|_{B \cup D_{2}}}(\delta)$ e concluímos este caso.

Por fim, é fácil ver que $\left.q\right|_{B \cup D_{2}} \leq p_{1}, p_{2}$.

O lema que acabamos de provar foi mostrado por Rabus [53] (Lema 5.3). Porém, o autor utiliza ali uma linguagem algébrica e nós optamos aqui pela linguagem topológica e por isso julgamos conveniente re-escrever a demonstração nesta linguagem.

\subsection{Os espaços topológicos genéricos $L$ e $K$}

Fixemos a partir de agora, e até o final desse capítulo, um modelo $V$ tal que $\theta, f \in V$ e um filtro $\mathbb{P}_{f}$-genérico $G$ sobre $V$.

Notemos que para cada $\xi<\eta<\theta$, segue do Lema 2.3 que o conjunto $\left\{p \in \mathbb{P}_{f}: \xi, \eta \in D_{p}\right\}$ é denso em $\mathbb{P}_{f}$. 
Definição 2.7. Para cada $\xi<\eta<\theta$ seja, em $V[G]$,

$$
h(\xi)=\bigcup_{p \in G} h_{p}(\xi) \quad e \quad i(\{\xi, \eta\})=\bigcup_{p \in G} i_{p}(\{\xi, \eta\}) .
$$

É fácil ver que para cada $\xi<\theta, \xi=\max h(\xi)$ e que se para quaisquer $\xi<\eta<\theta$, denotamos

$$
h(\xi) * h(\eta)= \begin{cases}h(\xi) \backslash h(\eta) & \text { se } \xi \in h(\eta), \\ h(\xi) \cap h(\eta) & \text { se } \xi \notin h(\eta),\end{cases}
$$

então $i(\{\xi, \eta\})$ é um subconjunto finito de $\xi$ tal que

$$
h(\xi) * h(\eta) \subseteq \bigcup_{\gamma \in i(\{\xi, \eta\})} h(\gamma)
$$

Em $V[G]$, seja $L$ o espaço topológico genérico $(\theta, \tau)$, onde $\tau$ é a topologia sobre $\theta$ que possui o conjunto

$$
\{h(\xi): \xi<\theta\} \cup\{L \backslash h(\xi): \xi<\theta\}
$$

como sub-base. Vamos chamar $h(\xi)$ de vizinhança genérica de $\xi$.

Temos o seguinte:

Proposição 2.8. Em $V[G]$, para todo $\xi<\theta, h(\xi)$ é compacto $e$

$$
\left\{h(\xi) \backslash \bigcup_{\eta \in F} h(\eta): F \in[\xi]^{<\aleph_{0}}\right\}
$$

forma um sistema fundamental de vizinhanças abertas de $\xi$.

Portanto, L é um espaço localmente compacto, disperso e zero-dimensional.

Demonstração. Segue diretamente da Proposição 1.24.

Notação 2.9. Em $V[G]$, denotamos a compactificação de Alexandroff de $L$ por $K$ e denotamos $\{*\}=K \backslash L$. 


\subsection{Os espaços de Banach $C_{0}(L)$ e $C(K)$}

Vejamos algumas das propriedades de $C_{0}(L)$ e de $C(K)$ :

Teorema 2.10. Em $V[G], L$ e $K$ são espaços dispersos e, portanto, $C_{0}(L)$ e $C(K)$ são espaços de Asplund.

Demonstração. Segue da Proposição 2.8 que $L$ e $K$ são espaços dispersos e segue do Teorema 1.43 que $C_{0}(L)$ e $C(K)$ são espaços de Asplund.

Teorema 2.11. Se $\theta=\omega_{1}$ ou $\omega_{2}$, então, em $V[G], L$ e $K$ têm peso $|\theta|$ e, portanto, $C_{0}(L)$ e $C(K)$ têm densidade $|\theta|$.

Demonstração. Para qualquer família $\mathcal{B}$ de abertos de $L$ da forma $h(\xi) \backslash \bigcup_{\gamma \in F} h(\gamma)$, onde $F \in[\xi]^{<\aleph_{0}}$ e $\xi<\theta$ tal que $|\mathcal{B}|<|\theta|$, temos que $\sup \bigcup \mathcal{B}<\theta$ e, portanto, $\mathcal{B}$ não pode ser uma base para a topologia de $L$. Por outro lado, pela Proposição 2.8, o conjunto

$$
\left\{h(\xi) \backslash \bigcup_{\gamma \in F} h(\gamma): \xi<\theta, F \in[\xi]^{<\aleph_{0}}\right\}
$$

é uma base topológica de $L$ de cardinalidade $|\theta|$. Logo, o peso de $L$ é $|\theta|$ e, como $K$ é a compactificação de Alexandroff de $L$, segue do Teorema 1.5 que $w(K)=w(L)=|\theta|$.

Por fim, temos, pelo Teorema 1.41, que a densidade de $C_{0}(L)$ e de $C(K)$ é $|\theta|$.

\subsection{Amalgamações}

Nesta seção, vamos provar dois lemas combinatórios que usaremos nos Capítulos 3 e 4 . Antes disso, vamos enunciar um lema de Juhasz e Soukup [33], cuja idéia está implícita em Rabus [53] e que vamos usar no Capítulo 3:

Lema 2.12 (Rabus, [53]; Juhasz, Soukup, [33]). Seja $t=\left(D_{t}, h_{t}, i_{t}\right) \in \mathbb{P}_{f}, D_{t}=T \cup E \cup F$, onde $T<E<F, E=\left\{\alpha_{1}<\cdots<\alpha_{k}\right\}, F=\left\{\alpha_{i}^{1}, \alpha_{i}^{2}: 1 \leq i \leq k\right\}, H \subseteq T e$ :

(i) para todo $1 \leq i \leq k$, temos que $h_{t}\left(\alpha_{i}^{1}\right) \cap h_{t}\left(\alpha_{i}^{2}\right)=\bigcup_{\xi \in H \cup E} h_{t}(\xi)$; 
(ii) para todo $1 \leq i \leq k$ e todo $\xi \in T$, temos que $f\left(\left\{\xi, \alpha_{i}\right\}\right)=f\left(\left\{\xi, \alpha_{i}^{1}\right\}\right)=f\left(\left\{\xi, \alpha_{i}^{2}\right\}\right)$.

Então existe $u=\left(D_{u}, h_{u}, i_{u}\right) \in \mathbb{P}_{f}$ tal que $D_{u}=T \cup E$ e:

(a) $u \leq\left. t\right|_{T}$;

(b) $u \leq\left. t\right|_{H \cup E}$;

(c) $T \backslash \bigcup_{\xi \in H \cup E} h_{t}(\xi) \subseteq h_{u}\left(\alpha_{1}\right)$.

Demonstração. Veja o Lema 2.16 de [33]. Observe que ali, $\theta=\omega_{2}$, mas não se usa esse fato na demonstração.

O primeiro lema combinatório que vamos provar, enunciado abaixo, tem hipóteses bastante fortes sobre duas condições do forcing $\mathbb{P}_{f}$ e, especificamente, sobre os domínios delas - a hipótese (C). Em contrapartida, garantimos a existência de uma amalgamação, uma condição mais forte que ambas, que mantém intacta uma das condições e que adiciona alguns pontos desta à vizinhança de certos pontos da outra. Pela hipótese (C), podemos chamar uma das condições de "condição menor" e a outra, de "condição maior". Nesses termos, dados um ponto $x$ da condição menor e um ponto $y$ da condição maior, obtemos uma amalgamação que adiciona $x$ à vizinhança de um ponto da condição maior se, e somente se, $y$ pertence a essa vizinhança.

Lema 2.13. Sejam $p_{1}=\left(D_{1}, h_{1}, i_{1}\right), p_{2}=\left(D_{2}, h_{2}, i_{2}\right) \in \mathbb{P}_{f},\left\{x_{1}^{1}, \ldots, x_{n}^{1}\right\} \subseteq D_{1} \backslash D_{2}$ e $\left\{x_{1}^{2}, \ldots, x_{n}^{2}\right\} \subseteq D_{2} \backslash D_{1}$ tais que para quaisquer $1 \leq i<j \leq n, x_{i}^{1} \neq x_{j}^{1}$ e $x_{i}^{2} \neq x_{j}^{2}$. Suponha que:

(A) se $\xi, \eta \in D_{1} \cap D_{2}$ e $\xi \neq \eta$, então $i_{1}(\{\xi, \eta\})=i_{2}(\{\xi, \eta\})$;

(B) se $\xi \in D_{1} \cap D_{2}$, então $h_{1}(\xi)=h_{2}(\xi)$;

(C) $D_{1} \cap D_{2}<D_{1} \backslash D_{2}<D_{2} \backslash D_{1}$;

(D) se $\xi \in D_{1} \backslash D_{2}$ e $\eta \in D_{2} \backslash D_{1}$, então $D_{1} \cap \xi \subseteq f(\{\xi, \eta\})$.

Então, existe $q \leq p_{1}, p_{2}$ em $\mathbb{P}_{f}$ tal que, para todo $\xi \in D_{2}$ e todo $1 \leq i \leq n$, temos que

$$
x_{i}^{1} \in h_{q}(\xi) \text { se, e somente se, } x_{i}^{2} \in h_{q}(\xi) .
$$


Demonstração. Definamos $q=\left(D_{q}, h_{q}, i_{q}\right)$ da seguinte forma: $D_{q}=D_{1} \cup D_{2}$;

$$
h_{q}(\xi)= \begin{cases}h_{1}(\xi) & \text { se } \xi \in D_{1}, \\ h_{2}(\xi) \cup\left\{x_{i}^{1}: x_{i}^{2} \in h_{2}(\xi) \text { e } 1 \leq i \leq n\right\} & \text { se } \xi \in D_{2} \backslash D_{1}\end{cases}
$$

e

$$
i_{q}(\{\xi, \eta\})= \begin{cases}i_{1}(\{\xi, \eta\}) & \text { se } \xi, \eta \in D_{1} \\ i_{2}(\{\xi, \eta\}) & \text { se } \xi, \eta \in D_{2} \\ f(\{\xi, \eta\}) \cap D_{q} & \text { caso contrário. }\end{cases}
$$

Observe que (A) implica que $i_{q}(\{\xi, \eta\})$ está bem-definido para quaisquer $\xi, \eta \in D_{1} \cap D_{2}$, $\xi \neq \eta$. Para os demais pares, é claro que $i_{q}$ está bem-definido.

Vejamos que $q \in \mathbb{P}_{f}$ : o fato que $q$ satisfaz as condições 1 e 3.(c) da Definição 2.1 segue diretamente da definição de $q$. Vejamos que $q$ satisfaz a condição 2 da Definição 2.1: seja $\xi \in D_{q}$ e considere os seguintes casos:

Caso 1. $\xi \in D_{1}$.

Neste caso, segue diretamente da definição de $q$ que $h_{1}(\xi)=h_{q}(\xi)$. Como $p_{1} \in \mathbb{P}_{f}$, temos que $\xi=\max h_{1}(\xi)=\max h_{q}(\xi)$.

Caso 2. $\xi \in D_{2} \backslash D_{1}$.

Aqui, segue da definição de $q$ que $h_{q}(\xi) \subseteq h_{2}(\xi) \cup D_{1}$ e $h_{2}(\xi) \subseteq h_{q}(\xi)$. Mas (C) implica que $D_{1}<\xi$. Assim, como $p_{2} \in \mathbb{P}_{f}$, temos que $\xi=\max h_{2}(\xi)=\max h_{q}(\xi)$.

Resta verificar que $q$ satisfaz as condições 3.(a) e (b) da Definição 2.1. Vejamos primeiramente o seguinte:

Afirmação 1. Para todo $\xi \in D_{2}$, temos que

$$
h_{q}(\xi) \cap D_{2}=h_{2}(\xi)
$$




$$
h_{q}(\xi) \cap\left(D_{1} \backslash D_{2}\right)=\left\{x_{i}^{1}: x_{i}^{2} \in h_{2}(\xi) \text { e } 1 \leq i \leq n\right\} .
$$

Demonstração da Afirmação 1. Seja $\xi \in D_{2}$ e considere os seguintes casos:

Caso 1. $\xi \in D_{1} \cap D_{2}$.

Aqui, temos pela definição que $h_{q}(\xi)=h_{1}(\xi)$ e segue de (B) que $h_{1}(\xi)=h_{2}(\xi)$. Como $h_{2}(\xi) \subseteq D_{2}$, temos que

$$
h_{q}(\xi) \cap D_{2}=h_{2}(\xi) \cap D_{2}=h_{2}(\xi)
$$

e temos a igualdade (I). Além disso, como $h_{q}(\xi)=h_{1}(\xi)=h_{2}(\xi)$, temos que $h_{q}(\xi) \cap\left(D_{1} \backslash\right.$ $\left.D_{2}\right)=\emptyset$. Mas para cada $1 \leq i \leq n$, temos, por hipótese, que $x_{i}^{2} \in D_{2} \backslash D_{1}$ e, segue de $(\mathrm{C})$ que $\xi<x_{i}^{2}$. Logo,

$$
h_{2}(\xi) \cap\left\{x_{i}^{2}: 1 \leq i \leq n\right\}=h_{1}(\xi) \cap\left\{x_{i}^{2}: 1 \leq i \leq n\right\} \subseteq D_{1} \cap\left\{x_{i}^{2}: 1 \leq i \leq n\right\}=\emptyset
$$

e, portanto,

$$
\left\{x_{i}^{1}: x_{i}^{2} \in h_{2}(\xi) \text { e } 1 \leq i \leq n\right\}=\emptyset
$$

concluindo (II).

Caso 2. $\xi \in D_{2} \backslash D_{1}$.

Neste caso, observe que $D_{2} \cap\left\{x_{i}^{1}: 1 \leq i \leq n\right\}=\emptyset$ e $\left(D_{1} \backslash D_{2}\right) \cap h_{2}(\xi)=\emptyset$. Daí, as igualdades (I) e (II) seguem facilmente da definição de $q$ e concluímos a demonstração da Afirmação 1.

Finalmente, provemos o seguinte:

Afirmação 2. q satisfaz as condições 3.(a) e (b) da Definição 2.1. 
Demonstração da Afirmação 2. Fixemos $\xi, \eta \in D_{q}, \xi<\eta$, e consideremos os seguintes casos:

Caso 1. $\xi, \eta \in D_{1}$.

Observe que, pela definição, $h_{q}(\xi)=h_{1}(\xi)$ e $h_{q}(\eta)=h_{1}(\eta)$. Assim, temos que se $\xi \in h_{q}(\eta)$, então $\xi \in h_{1}(\eta)$ e, portanto,

$$
h_{q}(\xi) \backslash h_{q}(\eta)=h_{1}(\xi) \backslash h_{1}(\eta)
$$

Por outro lado, se $\xi \notin h_{q}(\eta)$, então $\xi \notin h_{1}(\eta)$ e, portanto,

$$
h_{q}(\xi) \cap h_{q}(\eta)=h_{1}(\xi) \cap h_{1}(\eta) .
$$

Logo,

$$
h_{q}(\xi) * h_{q}(\eta)=h_{1}(\xi) * h_{1}(\eta)
$$

Mas como $i_{q}(\{\xi, \eta\})=i_{1}(\{\xi, \eta\}) \subseteq D_{1}$, segue da definição de $q$ que para todo $\gamma \in$ $i_{q}(\{\xi, \eta\}), h_{q}(\gamma)=h_{1}(\gamma)$. Daí, segue do fato que $p_{1} \in \mathbb{P}_{f}$ que

$$
h_{q}(\xi) * h_{q}(\eta)=h_{1}(\xi) * h_{1}(\eta) \subseteq \bigcup_{\gamma \in i_{1}(\{\xi, \eta\})} h_{1}(\gamma)=\bigcup_{\gamma \in i_{q}(\{\xi, \eta\})} h_{q}(\gamma) .
$$

Caso 2. $\xi, \eta \in D_{2}$.

Segue da igualdade (I) que $h_{q}(\xi) \cap D_{2}=h_{2}(\xi)$ e $h_{q}(\eta) \cap D_{2}=h_{2}(\eta)$. Daí, se $\xi \in h_{q}(\eta)$, também pela igualdade (I) temos que $\xi \in h_{2}(\eta)$ e, portanto,

$$
\left(h_{q}(\xi) \backslash h_{q}(\eta)\right) \cap D_{2}=\left(h_{q}(\xi) \cap D_{2}\right) \backslash\left(h_{q}(\eta) \cap D_{2}\right)=h_{2}(\xi) \backslash h_{2}(\eta)
$$

Analogamente, se $\xi \notin h_{q}(\eta)$, segue da igualdade (I) que $\xi \notin h_{2}(\eta)$ e, portanto,

$$
\left(h_{q}(\xi) \cap h_{q}(\eta)\right) \cap D_{2}=\left(h_{q}(\xi) \cap D_{2}\right) \cap\left(h_{q}(\eta) \cap D_{2}\right)=h_{2}(\xi) \cap h_{2}(\eta) .
$$

Logo,

$$
\left(h_{q}(\xi) * h_{q}(\eta)\right) \cap D_{2}=h_{2}(\xi) * h_{2}(\eta) .
$$


Mas pela definição de $q$, temos que $i_{q}(\{\xi, \eta\})=i_{2}(\{\xi, \eta\}) \subseteq D_{2}$. Daí, segue da definição de $q$ que para todo $\gamma \in i_{q}(\{\xi, \eta\}), h_{2}(\gamma) \subseteq h_{q}(\gamma)$. Logo, como $p_{2} \in \mathbb{P}_{f}$, temos que

$$
\left(h_{q}(\xi) * h_{q}(\eta)\right) \cap D_{2}=h_{2}(\xi) * h_{2}(\eta) \subseteq \bigcup_{\gamma \in i_{2}(\{\xi, \eta\})} h_{2}(\gamma) \subseteq \bigcup_{\gamma \in i_{q}(\{\xi, \eta\})} h_{q}(\gamma)
$$

Por outro lado, segue da igualdade (II) que

$$
h_{q}(\xi) \cap\left(D_{1} \backslash D_{2}\right)=\left\{x_{i}^{1}: x_{i}^{2} \in h_{2}(\xi) \text { e } 1 \leq i \leq n\right\}
$$

e

$$
h_{q}(\eta) \cap\left(D_{1} \backslash D_{2}\right)=\left\{x_{i}^{1}: x_{i}^{2} \in h_{2}(\eta) \text { e } 1 \leq i \leq n\right\} .
$$

Daí, se $\xi \in h_{q}(\eta)$, temos pela igualdade (I) que $\xi \in h_{2}(\eta)$ e, portanto,

$$
\begin{gathered}
\left(h_{q}(\xi) \backslash h_{q}(\eta)\right) \cap\left(D_{1} \backslash D_{2}\right)=\left(h_{q}(\xi) \cap\left(D_{1} \backslash D_{2}\right)\right) \backslash\left(h_{q}(\eta) \cap\left(D_{1} \backslash D_{2}\right)\right) \\
=\left\{x_{i}^{1}: x_{i}^{2} \in h_{2}(\xi) \text { e } 1 \leq i \leq n\right\} \backslash\left\{x_{i}^{1}: x_{i}^{2} \in h_{2}(\eta) \text { e } 1 \leq i \leq n\right\} \\
=\left\{x_{i}^{1}: x_{i}^{2} \in h_{2}(\xi) \backslash h_{2}(\eta) \text { e } 1 \leq i \leq n\right\}
\end{gathered}
$$

Analogamente, se $\xi \notin h_{q}(\eta)$, também pela igualdade (I) temos que $\xi \notin h_{2}(\eta)$ e, portanto,

$$
\begin{gathered}
\left(h_{q}(\xi) \cap h_{q}(\eta)\right) \cap\left(D_{1} \backslash D_{2}\right)=\left(h_{q}(\xi) \cap\left(D_{1} \backslash D_{2}\right)\right) \cap\left(h_{q}(\eta) \cap\left(D_{1} \backslash D_{2}\right)\right) \\
=\left\{x_{i}^{1}: x_{i}^{2} \in h_{2}(\xi) \text { e } 1 \leq i \leq n\right\} \cap\left\{x_{i}^{1}: x_{i}^{2} \in h_{2}(\eta) \text { e } 1 \leq i \leq n\right\} \\
=\left\{x_{i}^{1}: x_{i}^{2} \in h_{2}(\xi) \cap h_{2}(\eta) \text { e } 1 \leq i \leq n\right\} .
\end{gathered}
$$

Logo,

$$
\left(h_{q}(\xi) * h_{q}(\eta)\right) \cap\left(D_{1} \backslash D_{2}\right)=\left\{x_{i}^{1}: x_{i}^{2} \in h_{2}(\xi) * h_{2}(\eta) \text { e } 1 \leq i \leq n\right\}
$$

Assim, se $\zeta \in\left(h_{q}(\xi) * h_{q}(\eta)\right) \cap\left(D_{1} \backslash D_{2}\right)$, então existe $1 \leq i \leq n$ tal que $x_{i}^{2} \in h_{2}(\xi) * h_{2}(\eta)$ e $\zeta=x_{i}^{1}$. Como $p_{2} \in \mathbb{P}_{f}$, existe $\gamma \in i_{2}(\{\xi, \eta\})=i_{q}(\{\xi, \eta\})$ tal que $x_{i}^{2} \in h_{2}(\gamma)$. Mas $x_{i}^{2} \in D_{2} \backslash D_{1}$ e $x_{i}^{2} \leq \gamma$ e, então, segue de (C) que $\gamma \in D_{2} \backslash D_{1}$. Daí, segue da igualdade (II) que $x_{i}^{1} \in h_{q}(\gamma)$ e concluímos, assim, a demonstração para este caso. 
Caso 3. $\xi \in D_{1} \backslash D_{2}$ e $\eta \in D_{2} \backslash D_{1}$.

Neste caso, fixe $\zeta \in D_{q}$ tal que $\zeta \in h_{q}(\xi) * h_{q}(\eta)$. Como $\xi \in D_{1}$ e $\zeta<\xi$, segue de (C) que $\zeta \in D_{1}$. Mas segue de (D) que $\zeta \in D_{1} \cap \xi \subseteq D_{q} \cap f(\{\xi, \eta\})=i_{q}(\{\xi, \eta\})$ e, portanto,

$$
\zeta \in h_{q}(\zeta) \subseteq \bigcup_{\left.\gamma \in i_{q}(\xi, \eta\}\right)} h_{q}(\gamma),
$$

concluindo a demonstração para este caso.

Observemos que (C) implica que o caso $\xi \in D_{2} \backslash D_{1}$ e $\eta \in D_{1} \backslash D_{2}$ não pode ocorrer, pois assumimos que $\xi<\eta$ e concluímos, assim, a demonstração da Afirmação 2 e do fato que $q \in \mathbb{P}_{f}$.

Finalmente, é fácil ver que $q \leq p_{1}$, diretamente pela definição de $q$. O fato que $q \leq p_{2}$ segue também facilmente da definição de $q$ e da Afirmação 1.

Por fim, fixe $\xi \in D_{2}$ e $1 \leq i \leq n$. Como $x_{i}^{2} \in D_{2}$, segue da igualdade (I) que

$$
x_{i}^{2} \in h_{q}(\xi) \text { se, e somente se, } x_{i}^{2} \in h_{2}(\xi) .
$$

Por outro lado, como $x_{i}^{1} \in D_{1} \backslash D_{2}$, segue da igualdade (II) que

$$
x_{i}^{1} \in h_{q}(\xi) \text { se, e somente se, } x_{i}^{2} \in h_{2}(\xi)
$$

Portanto,

$$
x_{i}^{1} \in h_{q}(\xi) \text { se, e somente se, } x_{i}^{2} \in h_{q}(\xi)
$$

concluindo a demonstração do resultado.

Finalmente, chegamos ao segundo lema combinatório: queremos amalgamar duas condições numa maneira parecida à do lema anterior, pois queremos "copiar" algumas coisas que acontecem com pontos de uma condição para pontos correspondentes da outra condição. Porém, sem a hipótese $(\mathrm{C})$ do lema anterior (que substituímos pela hipótese mais fraca 
(D) no lema abaixo), as coisas se complicam ainda mais. Antes não precisávamos nos preocupar com pontos da condição menor que estivessem acima de pontos da maior, já que eles não existiam. Por outro lado, tendo em vista que queremos fazer algumas "cópias", a amalgamação construída no Lema 2.7 de [33] para mostrar que o forcing é c.c.c. também não nos ajuda, já que essa amalgamação altera as vizinhanças dos pontos o mínimo possível.

A solução que encontramos é construir uma amalgamação que aproveita metade de cada idéia (observe que aqui, pela condição (D) também está claro que podemos chamar uma das condições de "condição menor" e a outra, de "condição maior"): para os pontos da condição menor, usamos a idéia de [33], adicionando à sua vizinhança apenas os pontos da condição maior estritamente necessários; para os da maior, adicionamos à sua vizinhança os pontos da condição menor cuja "cópia" na maior pertence à sua vizinhança.

Convém notar que para obter condições satisfazendo a hipótese (D) do lema anterior (2.13) e a hipótese (E).(iii) do lema abaixo (2.14), precisaremos de uma hipótese sobre a função $f$ que é mais forte que a propriedade $\Delta$ utilizada em [33] e [53]. As funções utilizadas nos Capítulos 3 e 4 garantirão a existência destas condições, quando necessário.

Lema 2.14. Sejam $p_{1}=\left(D_{1}, h_{1}, i_{1}\right), p_{2}=\left(D_{2}, h_{2}, i_{2}\right) \in \mathbb{P}_{f}$ duas condições tais que existe uma função bijetora e $: D_{1} \rightarrow D_{2}$ que preserva ordem satisfazendo as seguintes condições:

(A) se $\xi, \eta \in D_{1} \cap D_{2}$ e $\xi \neq \eta$, então $i_{1}(\{\xi, \eta\})=i_{2}(\{\xi, \eta\})$;

(B) se $\xi, \eta \in D_{1}$, então $\xi \in h_{1}(\eta)$ se, e somente se, $e(\xi) \in h_{2}(e(\eta))$;

(C) se $\xi \in D_{1} \cap D_{2}$, então $e(\xi)=\xi$;

(D) se $\xi \in D_{1}$, então $\xi \leq e(\xi)$;

(E) para todo $\zeta \in D_{1} \cap D_{2}$, todo $\xi \in D_{1} \backslash D_{2}$ e todo $\eta \in D_{2} \backslash D_{1}$ :

(i) se $\zeta<\xi$, então $f(\{\zeta, \eta\}) \subseteq f(\{\xi, \eta\})$;

(ii) $D_{1} \cap \xi \cap \eta \subseteq f(\{\xi, \eta\})$.

Então existe $q \in \mathbb{P}_{f}, q \leq p_{1}, p_{2}$ tal que para todo $\xi \in D_{1}$ e todo $\eta \in D_{2}$,

$$
\xi \in h_{q}(\eta) \text { se, e somente se, } e(\xi) \in h_{2}(\eta) \text {. }
$$


Demonstração. Para cada $\eta \in D_{2} \backslash D_{1}$, se o conjunto $\left\{\delta \in D_{1} \cap D_{2}: \eta \in h_{2}(\delta)\right\}$ é não-vazio, defina

$$
\delta_{\eta}=\min \left\{\delta \in D_{1} \cap D_{2}: \eta \in h_{2}(\delta)\right\} .
$$

Observe que, para todo $\eta \in D_{2} \backslash D_{1}$, se $\delta_{\eta}$ existe, então $\eta \leq \delta_{\eta}$, pois $\eta \in h_{2}\left(\delta_{\eta}\right)$. Como $\eta \notin D_{1}$ e $\delta_{\eta} \in D_{1} \cap D_{2}$, temos que $\eta \neq \delta_{\eta}$ e, portanto, $\eta<\delta_{\eta}$.

Defina $q=\left(D_{q}, h_{q}, i_{q}\right)$ por: $D_{q}=D_{1} \cup D_{2}$;

$$
h_{q}(\xi)= \begin{cases}h_{1}(\xi) \cup h_{2}(\xi) & \text { se } \xi \in D_{1} \cap D_{2}, \\ h_{1}(\xi) \cup\left\{\eta \in D_{2} \backslash D_{1}: \delta_{\eta} \text { existe e } \delta_{\eta} \in h_{1}(\xi)\right\} & \text { se } \xi \in D_{1} \backslash D_{2} \\ h_{2}(\xi) \cup\left\{\eta \in D_{1} \backslash D_{2}: e(\eta) \in h_{2}(\xi)\right\} & \text { se } \xi \in D_{2} \backslash D_{1}\end{cases}
$$

e

$$
i_{q}(\{\xi, \eta\})= \begin{cases}i_{1}(\{\xi, \eta\}) & \text { se } \xi, \eta \in D_{1} \\ i_{2}(\{\xi, \eta\}) & \text { se } \xi, \eta \in D_{2} \\ f(\{\xi, \eta\}) \cap D_{q} & \text { caso contrário. }\end{cases}
$$

Notemos que (A) implica que $i_{q}(\{\xi, \eta\})$ está bem-definido para quaisquer $\xi, \eta \in D_{1} \cap D_{2}$, $\xi \neq \eta$ e é claro que $i_{q}$ está bem-definido para os demais pares.

Precisamos mostrar que $q \in \mathbb{P}_{f}$, ou seja, que $q$ satisfaz as condições 1,2 e 3 da Definição 2.1. O fato que $q$ satisfaz as condições 1 e 3.(c) da Definição 2.1 segue diretamente da definição de $q$ e do fato que $p_{1}, p_{2} \in \mathbb{P}_{f}$. Antes de mostrar que $q$ satisfaz as demais condições da Definição 2.1, provemos algumas afirmações:

Afirmação 1. Para todo $\xi \in D_{1}$, temos que

$$
h_{q}(\xi) \cap D_{1}=h_{1}(\xi)
$$

e

$$
h_{q}(\xi) \cap\left(D_{2} \backslash D_{1}\right)=\left\{\eta \in D_{2} \backslash D_{1}: \delta_{\eta} \text { existe e } \delta_{\eta} \in h_{1}(\xi)\right\} .
$$

Demonstração da Afirmação 1. Mostremos, primeiramente, a igualdade (I): fixe $\xi \in D_{1}$ e considere os seguintes casos: 
Caso 1. $\xi \in D_{1} \backslash D_{2}$.

Neste caso, como $\left\{\zeta \in D_{2} \backslash D_{1}: \delta_{\zeta}\right.$ existe e $\left.\delta_{\zeta} \in h_{1}(\xi)\right\} \cap D_{1}=\emptyset$, a igualdade (I) segue facilmente da definição de $q$.

Caso 2. $\xi \in D_{1} \cap D_{2}$.

Neste caso, segue da definição de $q$ que $h_{1}(\xi) \subseteq h_{q}(\xi)$ e segue do fato que $p_{1} \in \mathbb{P}_{f}$ que $h_{1}(\xi) \subseteq D_{1}$. Logo, $h_{1}(\xi) \subseteq h_{q}(\xi) \cap D_{1}$. Para verificar a outra inclusão, seja $\eta \in h_{q}(\xi) \cap D_{1}$. Daí, $\eta \in h_{1}(\xi)$ ou $\eta \in h_{2}(\xi)$. Se $\eta \in h_{1}(\xi)$, concluímos a demonstração da igualdade (I). Se $\eta \in h_{2}(\xi) \cap D_{1}$, então $\eta \in D_{1} \cap D_{2}$. Portanto, (C) implica que $e(\eta)=\eta$. Mas neste caso (C) também implica que $e(\xi)=\xi$. Daí, segue de (B) que $\eta=e(\eta) \in h_{1}(e(\xi))=h_{1}(\xi)$, concluindo a demonstração da igualdade (I).

Mostremos agora a igualdade (II): fixe $\xi \in D_{1}$ e considere novamente os seguintes casos: Caso 1. $\xi \in D_{1} \backslash D_{2}$.

Neste caso, a igualdade (II) segue diretamente da definição de $q$ e do fato que $h_{1}(\xi) \cap$ $\left(D_{2} \backslash D_{1}\right)=\emptyset$.

Caso 2. $\xi \in D_{1} \cap D_{2}$.

Mostremos as duas inclusões.

Primeiramente, seja $\eta \in h_{q}(\xi) \cap\left(D_{2} \backslash D_{1}\right)$. Observe que $\eta \notin h_{1}(\xi)$, já que $h_{1}(\xi) \subseteq D_{1}$ e $\eta \notin D_{1}$. Logo, $\eta \in h_{2}(\xi) \backslash h_{1}(\xi)$. Como $\xi \in D_{1} \cap D_{2}$, temos que $\delta_{\eta}$ existe e, pela minimalidade de $\delta_{\eta}, \delta_{\eta} \leq \xi$. Suponhamos, por absurdo, que $\delta_{\eta} \notin h_{1}(\xi)$. Então, $\delta_{\eta}<\xi$ e segue de (B) que $\delta_{\eta} \notin h_{2}(e(\xi))$. Lembramos que neste caso (C) implica que $e(\xi)=\xi$ e, portanto, temos que $\delta_{\eta} \notin h_{2}(\xi)$. Daí, $\eta \in h_{2}\left(\delta_{\eta}\right) \cap h_{2}(\xi)=h_{2}\left(\delta_{\eta}\right) * h_{2}(\xi)$. Logo, existe $\delta \in i_{2}\left(\left\{\delta_{\eta}, \xi\right\}\right)$ tal que $\eta \in h_{2}(\delta)$. Mas segue de (A) que $i_{1}\left(\left\{\delta_{\eta}, \xi\right\}\right)=i_{2}\left(\left\{\delta_{\eta}, \xi\right\}\right)$. Daí, como $p_{1}, p_{2} \in \mathbb{P}_{f}$, temos que $i_{2}\left(\left\{\delta_{\eta}, \xi\right\}\right) \subseteq D_{1} \cap D_{2} \cap f\left(\left\{\delta_{\eta}, \xi\right\}\right)$. Lembrando que $f\left(\left\{\delta_{\eta}, \xi\right\}\right) \subseteq \min \left\{\delta_{\eta}, \xi\right\}$, temos que $\delta \in D_{1} \cap D_{2} \cap \delta_{\eta}$. Assim, temos que $\eta \in h_{2}(\delta), \delta \in D_{1} \cap D_{2}$ e $\delta<\delta_{\eta}$, contradizendo a minimalidade de $\delta_{\eta}$.

Seja, agora, $\eta \in D_{2} \backslash D_{1}$ tal que $\delta_{\eta}$ existe e $\delta_{\eta} \in h_{1}(\xi)$. Temos duas possibilidades: $\delta_{\eta}=\xi$ ou $\delta_{\eta}<\xi$. Se $\delta_{\eta}=\xi$, temos que $\eta \in h_{2}\left(\delta_{\eta}\right)=h_{2}(\xi)$ e concluímos a demonstração. Se 
$\delta_{\eta}<\xi$, segue de (B) que $\delta_{\eta} \in h_{2}(e(\xi))$. Lembramos que (C) implica que $e(\xi)=\xi$ e, portanto, temos que $\delta_{\eta} \in h_{2}(\xi)$. Então, $h_{2}\left(\delta_{\eta}\right) * h_{2}(\xi)=h_{2}\left(\delta_{\eta}\right) \backslash h_{2}(\xi)$. Suponhamos, por absurdo, que $\eta \notin h_{2}(\xi)$. Logo, $\eta \in h_{2}\left(\delta_{\eta}\right) * h_{2}(\xi)$ e daí, existe $\delta \in i_{2}\left(\left\{\delta_{\eta}, \xi\right\}\right)$ tal que $\eta \in h_{2}(\delta)$. Novamente, segue de $(\mathrm{A})$ que $i_{2}\left(\left\{\delta_{\eta}, \xi\right\}\right) \subseteq D_{1} \cap D_{2} \cap \delta_{\eta}$ e, portanto, $\delta \in D_{1} \cap D_{2} \cap \delta_{\eta}$. Assim, temos que $\eta \in h_{2}(\delta), \delta \in D_{1} \cap D_{2}$ e $\delta<\delta_{\eta}$, contradizendo a minimalidade de $\delta_{\eta}$.

Concluímos, assim, a demonstração deste caso, da igualdade (II) e da Afirmação 1.

Mostremos agora uma afirmação correspondente à Afirmação 1, mas para $\xi \in D_{2}$ :

Afirmação 2. Para todo $\xi \in D_{2}$, temos que

$$
h_{q}(\xi) \cap D_{2}=h_{2}(\xi)
$$

$e$

$$
h_{q}(\xi) \cap\left(D_{1} \backslash D_{2}\right)=\left\{\eta \in D_{1} \backslash D_{2}: e(\eta) \in h_{2}(\xi)\right\}
$$

Demonstração da Afirmação 2. Mostremos primeiramente a igualdade (III): fixe $\xi \in D_{2}$ e considere os seguintes casos:

Caso 1. $\xi \in D_{2} \backslash D_{1}$.

Neste caso, a igualdade (III) segue diretamente da definição de $q$ e do fato que $\{\zeta \in$ $\left.D_{1} \backslash D_{2}: e(\zeta) \in h_{2}(\xi)\right\} \cap D_{2}=\emptyset$.

Caso 2. $\xi \in D_{1} \cap D_{2}$.

Neste caso, segue da definição de $q$ e do fato que $h_{2}(\xi) \subseteq D_{2}$ que $h_{2}(\xi) \subseteq h_{q}(\xi) \cap D_{2}$. Para mostrar a outra inclusão, fixe $\eta \in h_{q}(\xi) \cap D_{2}$ e temos então que $\eta \in h_{1}(\xi)$ ou $\eta \in h_{2}(\xi)$. Se $\eta \in h_{2}(\xi)$, concluímos a demonstração da igualdade (III). Se $\eta \in h_{1}(\xi) \cap D_{2}$, temos que $\eta \in h_{1}(\xi) \cap D_{2} \subseteq D_{1} \cap D_{2}$. Daí, (C) implica que $e(\eta)=\eta$. Mas (C) também implica que $e(\xi)=\xi$. Segue então do fato que $\eta \in h_{1}(\xi)$ e de (B) que $\eta=e(\eta) \in h_{2}(e(\xi))=h_{2}(\xi)$, 
concluindo a demonstração da igualdade (III).

Mostremos, finalmente, a igualdade (IV): fixe $\xi \in D_{2}$ e considere os seguintes casos: Caso 1. $\xi \in D_{2} \backslash D_{1}$.

Neste caso, a igualdade (III) segue diretamente da definição de $q$ e do fato que $h_{2}(\xi) \cap$ $\left(D_{1} \backslash D_{2}\right)=\emptyset$.

Caso 2. $\xi \in D_{1} \cap D_{2}$.

Neste caso, segue de (C) que $e(\xi)=\xi$. Tomemos $\eta \in D_{1} \backslash D_{2}$. Daí, se $\eta \in h_{q}(\xi) \cap\left(D_{1} \backslash D_{2}\right)$, como $h_{2}(\xi) \subseteq D_{2}$, temos que $\eta \in h_{1}(\xi)$ e, segue de $(\mathrm{B})$, que $e(\eta) \in h_{2}(e(\xi))=h_{2}(\xi)$. Por outro lado, se $e(\eta) \in h_{2}(\xi)$, segue novamente de (B) que $\eta \in h_{1}(\xi)$ e concluímos a demonstração deste caso, da igualdade (IV) e da Afirmação 2.

Voltemos agora à demonstração do lema. Lembramos que para mostrar que $q \in \mathbb{P}_{f}$, resta mostrar que $q$ satisfaz as condições 2, 3.(a) e 3.(b) da Definição 2.1.

Afirmação 3. q satisfaz a condição 2 da Definição 2.1.

Demonstração da Afirmação 3. Fixe $\xi \in D_{q}$ e considere os seguintes casos:

Caso 1. $\xi \in D_{1}$.

Seja $\eta \in h_{q}(\xi)$. Pela Afirmação 1, temos que $\eta \in h_{1}(\xi)$ ou $\eta \in D_{2} \backslash D_{1}$ e $\delta_{\eta} \in h_{1}(\xi)$. Se $\eta \in h_{1}(\xi)$, como $p_{1} \in \mathbb{P}_{f}$, temos que $\eta \leq \max h_{1}(\xi)=\xi$. Se $\eta \in D_{2} \backslash D_{1}$ e $\delta_{\eta} \in h_{1}(\xi)$, então $\eta \leq \delta_{\eta} \leq \xi$. Como $\xi \in h_{1}(\xi) \subseteq h_{q}(\xi)$, segue que $\xi=\max h_{q}(\xi)$.

Caso 2. $\xi \in D_{2} \backslash D_{1}$.

Seja $\eta \in h_{q}(\xi)$. Pela Afirmação 2, temos que $\eta \in h_{2}(\xi)$ ou $\eta \in D_{1} \backslash D_{2}$ e $e(\eta) \in h_{2}(\xi)$. Se $\eta \in h_{2}(\xi)$, como $p_{2} \in \mathbb{P}_{f}$, temos que $\eta \leq \max h_{2}(\xi)=\xi$. Se $\eta \in D_{1} \backslash D_{2}$ e $e(\eta) \in h_{2}(\xi)$, então segue de (D) que $\eta \leq e(\eta) \leq \xi$. Como $\xi \in h_{2}(\xi) \subseteq h_{q}(\xi)$, segue que $\xi=\max h_{q}(\xi)$.

Concluímos, assim, a demonstração da Afirmação 3. 
Para concluir que $q \in \mathbb{P}_{f}$, resta provar que $q$ satisfaz as condições 3.(a) e (b) da Definição 2.1:

Afirmação 4. q satisfaz as condições 3.(a) e (b) da Definição 2.1.

Demonstração da Afirmação 4. Sejam $\xi, \eta \in D_{q}, \xi<\eta$ e consideremos os seguintes casos:

Caso 1. $\xi, \eta \in D_{1}$.

Neste caso, segue da igualdade (I) que $h_{q}(\eta) \cap D_{1}=h_{1}(\eta)$ e $h_{q}(\xi) \cap D_{1}=h_{1}(\xi)$.

Assim, se $\xi \in h_{q}(\eta)$, então

$$
\left(h_{q}(\xi) \backslash h_{q}(\eta)\right) \cap D_{1}=\left(h_{q}(\xi) \cap D_{1}\right) \backslash\left(h_{q}(\eta) \cap D_{1}\right)=h_{1}(\xi) \backslash h_{1}(\eta)
$$

Por outro lado, se $\xi \notin h_{q}(\eta)$, então

$$
\left(h_{q}(\xi) \cap h_{q}(\eta)\right) \cap D_{1}=\left(h_{q}(\xi) \cap D_{1}\right) \cap\left(h_{q}(\eta) \cap D_{1}\right)=h_{1}(\xi) \cap h_{1}(\eta) .
$$

Como, também pela igualdade (I), $\xi \in h_{q}(\eta)$ se, e somente se, $\xi \in h_{1}(\eta)$, segue que $\left(h_{q}(\xi) * h_{q}(\eta)\right) \cap D_{1}=h_{1}(\xi) * h_{1}(\eta)$. Daí, como $i_{1}(\{\xi, \eta\})=i_{q}(\{\xi, \eta\})$ e para todo $\gamma \in D_{1}$ temos que $h_{1}(\gamma) \subseteq h_{q}(\gamma)$, segue do fato que $p_{1} \in \mathbb{P}_{f}$ que

$$
\left(h_{q}(\xi) * h_{q}(\eta)\right) \cap D_{1}=h_{1}(\xi) * h_{1}(\eta) \subseteq \bigcup_{\gamma \in i_{1}(\{\xi, \eta\})} h_{1}(\gamma) \subseteq \bigcup_{\gamma \in i_{q}(\{\xi, \eta\})} h_{q}(\gamma)
$$

Além disso, segue da igualdade (II) que

$$
h_{q}(\xi) \cap\left(D_{2} \backslash D_{1}\right)=\left\{\zeta \in D_{2} \backslash D_{1}: \delta_{\zeta} \text { existe e } \delta_{\zeta} \in h_{1}(\xi)\right\}
$$

$\mathrm{e}$

$$
h_{q}(\eta) \cap\left(D_{2} \backslash D_{1}\right)=\left\{\zeta \in D_{2} \backslash D_{1}: \delta_{\zeta} \text { existe e } \delta_{\zeta} \in h_{1}(\eta)\right\} .
$$

Assim, se $\xi \in h_{q}(\eta)$, então

$$
\left(h_{q}(\xi) \backslash h_{q}(\eta)\right) \cap\left(D_{2} \backslash D_{1}\right)=\left(h_{q}(\xi) \cap\left(D_{2} \backslash D_{1}\right)\right) \backslash\left(h_{q}(\eta) \cap\left(D_{2} \backslash D_{1}\right)\right)
$$




$$
\begin{gathered}
=\left\{\zeta \in D_{2} \backslash D_{1}: \delta_{\zeta} \text { existe e } \delta_{\zeta} \in h_{1}(\xi)\right\} \backslash\left\{\zeta \in D_{2} \backslash D_{1}: \delta_{\zeta} \text { existe e } \delta_{\zeta} \in h_{1}(\eta)\right\} \\
=\left\{\zeta \in D_{2} \backslash D_{1}: \delta_{\zeta} \text { existe e } \delta_{\zeta} \in h_{1}(\xi) \backslash h_{1}(\eta)\right\}
\end{gathered}
$$

Por outro lado, se $\xi \notin h_{q}(\eta)$, então

$$
\begin{gathered}
\left(h_{q}(\xi) \cap h_{q}(\eta)\right) \cap\left(D_{2} \backslash D_{1}\right)=\left(h_{q}(\xi) \cap\left(D_{2} \backslash D_{1}\right)\right) \cap\left(h_{q}(\eta) \cap\left(D_{2} \backslash D_{1}\right)\right) \\
=\left\{\zeta \in D_{2} \backslash D_{1}: \delta_{\zeta} \text { existe e } \delta_{\zeta} \in h_{1}(\xi)\right\} \cap\left\{\zeta \in D_{2} \backslash D_{1}: \delta_{\zeta} \text { existe e } \delta_{\zeta} \in h_{1}(\eta)\right\} \\
=\left\{\zeta \in D_{2} \backslash D_{1}: \delta_{\zeta} \text { existe e } \delta_{\zeta} \in h_{1}(\xi) \cap h_{1}(\eta)\right\}
\end{gathered}
$$

Como, pela igualdade (I), $\xi \in h_{q}(\eta)$ se, e somente se, $\xi \in h_{1}(\eta)$, segue que

$$
\left(h_{q}(\xi) * h_{q}(\eta)\right) \cap\left(D_{2} \backslash D_{1}\right)=\left\{\zeta \in D_{2} \backslash D_{1}: \delta_{\zeta} \text { existe e } \delta_{\zeta} \in h_{1}(\xi) * h_{1}(\eta)\right\}
$$

Daí, se $\zeta \in\left(h_{q}(\xi) * h_{q}(\eta)\right) \cap D_{2} \backslash D_{1}$, então $\delta_{\zeta}$ existe e existe $\gamma \in i_{1}(\{\xi, \eta\})=i_{q}(\{\xi, \eta\})$ tal que $\delta_{\zeta} \in h_{1}(\gamma)$. Como $\gamma \in i_{1}(\{\xi, \eta\}) \subseteq D_{1}$, segue da igualdade (II) que $\zeta \in h_{q}(\gamma)$. Ou seja, temos que

$$
\left(h_{q}(\xi) * h_{q}(\eta)\right) \cap D_{2} \backslash D_{1} \subseteq \bigcup_{\gamma \in i_{q}(\{\xi, \eta\})} h_{q}(\gamma)
$$

e concluímos a demonstração das condições 3.(a) e (b) da Definição 2.1 para este caso.

Caso 2. $\xi, \eta \in D_{2}$.

Segue da igualdade (III) que $h_{q}(\eta) \cap D_{2}=h_{2}(\eta)$ e $h_{q}(\xi) \cap D_{2}=h_{2}(\xi)$.

Assim, se $\xi \in h_{q}(\eta)$, então

$$
\left(h_{q}(\xi) \backslash h_{q}(\eta)\right) \cap D_{2}=\left(h_{q}(\xi) \cap D_{2}\right) \backslash\left(h_{q}(\eta) \cap D_{2}\right)=h_{2}(\xi) \backslash h_{2}(\eta) .
$$

Por outro lado, se $\xi \notin h_{q}(\eta)$, então

$$
\left(h_{q}(\xi) \cap h_{q}(\eta)\right) \cap D_{2}=\left(h_{q}(\xi) \cap D_{2}\right) \cap\left(h_{q}(\eta) \cap D_{2}\right)=h_{2}(\xi) \cap h_{2}(\eta) .
$$

Como, também pela igualdade (III), $\xi \in h_{q}(\eta)$ se, e somente se, $\xi \in h_{2}(\eta)$, segue que $\left(h_{q}(\xi) * h_{q}(\eta)\right) \cap D_{2}=h_{2}(\xi) * h_{2}(\eta)$. Daí, como $i_{2}(\{\xi, \eta\})=i_{q}(\{\xi, \eta\})$ e para todo $\gamma \in D_{2}$ 
temos que $h_{2}(\gamma) \subseteq h_{q}(\gamma)$, segue do fato que $p_{2} \in \mathbb{P}_{f}$ que

$$
\left(h_{q}(\xi) * h_{q}(\eta)\right) \cap D_{2}=h_{2}(\xi) * h_{2}(\eta) \subseteq \bigcup_{\gamma \in i_{2}(\{\xi, \eta\})} h_{2}(\gamma) \subseteq \bigcup_{\gamma \in i_{q}(\{\xi, \eta\})} h_{q}(\gamma)
$$

Além disso, segue da igualdade (IV) que

$$
h_{q}(\xi) \cap\left(D_{1} \backslash D_{2}\right)=\left\{\zeta \in D_{1} \backslash D_{2}: e(\zeta) \in h_{2}(\xi)\right\}
$$

e

$$
h_{q}(\eta) \cap\left(D_{1} \backslash D_{2}\right)=\left\{\zeta \in D_{1} \backslash D_{2}: e(\zeta) \in h_{2}(\eta)\right\} .
$$

Assim, se $\xi \in h_{q}(\eta)$, então

$$
\begin{gathered}
\left(h_{q}(\xi) \backslash h_{q}(\eta)\right) \cap\left(D_{1} \backslash D_{2}\right)=\left(h_{q}(\xi) \cap\left(D_{1} \backslash D_{2}\right)\right) \backslash\left(h_{q}(\eta) \cap\left(D_{1} \backslash D_{2}\right)\right) \\
=\left\{\zeta \in D_{1} \backslash D_{2}: e(\zeta) \in h_{2}(\xi)\right\} \backslash\left\{\zeta \in D_{1} \backslash D_{2}: e(\zeta) \in h_{2}(\eta)\right\} \\
=\left\{\zeta \in D_{1} \backslash D_{2}: e(\zeta) \in h_{2}(\xi) \backslash h_{2}(\eta)\right\}
\end{gathered}
$$

Por outro lado, se $\xi \notin h_{q}(\eta)$, então

$$
\begin{gathered}
\left(h_{q}(\xi) \cap h_{q}(\eta)\right) \cap\left(D_{1} \backslash D_{2}\right)=\left(h_{q}(\xi) \cap\left(D_{1} \backslash D_{2}\right)\right) \cap\left(h_{q}(\eta) \cap\left(D_{1} \backslash D_{2}\right)\right) \\
=\left\{\zeta \in D_{1} \backslash D_{2}: e(\zeta) \in h_{2}(\xi)\right\} \cap\left\{\zeta \in D_{1} \backslash D_{2}: e(\zeta) \in h_{2}(\eta)\right\} \\
=\left\{\zeta \in D_{1} \backslash D_{2}: e(\zeta) \in h_{2}(\xi) \cap h_{2}(\eta)\right\}
\end{gathered}
$$

Como, também pela igualdade (III), $\xi \in h_{q}(\eta)$ se, e somente se, $\xi \in h_{2}(\eta)$, segue que

$$
\left(h_{q}(\xi) * h_{q}(\eta)\right) \cap\left(D_{1} \backslash D_{2}\right)=\left\{\zeta \in D_{1} \backslash D_{2}: e(\zeta) \in h_{2}(\xi) * h_{2}(\eta)\right\}
$$

Daí, se $\zeta \in\left(h_{q}(\xi) * h_{q}(\eta)\right) \cap D_{1} \backslash D_{2}$, existe $\gamma \in i_{2}(\{\xi, \eta\})=i_{q}(\{\xi, \eta\})$ tal que $e(\zeta) \in h_{2}(\gamma)$. Como $\gamma \in i_{2}(\{\xi, \eta\}) \subseteq D_{2}$, segue da igualdade (IV) que $\zeta \in h_{q}(\gamma)$. Portanto, temos que

$$
\left(h_{q}(\xi) * h_{q}(\eta)\right) \cap D_{1} \backslash D_{2} \subseteq \bigcup_{\gamma \in i_{q}(\{\xi, \eta\})} h_{q}(\gamma)
$$


e concluímos a demonstração das condições 3.(a) e (b) da Definição 2.1 para este caso.

Caso 3. $\xi \in D_{1} \backslash D_{2}$ e $\eta \in D_{2} \backslash D_{1}$.

Fixemos $\zeta \in h_{q}(\xi) * h_{q}(\eta)$ e consideremos os seguintes subcasos:

Subcaso 3.1. $\zeta \in D_{1}$.

Aqui, temos que $\zeta \in D_{1} \cap \xi \cap \eta$ e, por (E).(ii), temos que $\zeta \in f(\{\xi, \eta\})$. Como $\zeta \in$ $D_{1} \subseteq D_{q}$, segue da definição de $q$ que $\zeta \in i_{q}(\{\xi, \eta\})$. Portanto, tomando $\gamma=\zeta$ temos que $\zeta \in h_{q}(\gamma)$ e $\gamma \in i_{q}(\{\xi, \eta\})$.

Subcaso 3.2. $\zeta \in D_{2} \backslash D_{1}$.

Neste caso, segue da igualdade (II) que $\delta_{\zeta}$ existe e $\delta_{\zeta} \in h_{1}(\xi)$. Logo, $\delta_{\zeta} \in D_{1} \cap \xi \cap \eta$ e, novamente segue de (E).(ii) que $\delta_{\zeta} \in f(\{\xi, \eta\}) \cap D_{q}=i_{q}(\{\xi, \eta\})$. Daí, pela definição de $\delta_{\zeta}$ temos que $\zeta \in h_{2}\left(\delta_{\zeta}\right) \subseteq h_{q}\left(\delta_{\zeta}\right)$. Tomando $\gamma=\delta_{\zeta}$, temos que $\zeta \in h_{q}(\gamma)$ e $\gamma \in i_{q}(\{\xi, \eta\})$, concluindo a demonstração para este caso.

Caso 4. $\xi \in D_{2} \backslash D_{1}$ e $\eta \in D_{1} \backslash D_{2}$.

Novamente, fixemos $\zeta \in h_{q}(\xi) * h_{q}(\eta)$ e consideremos os seguintes subcasos:

Subcaso 4.1. $\zeta \in D_{1}$.

Temos aqui que $\zeta \in D_{1} \cap \xi \cap \eta$ e, segue de (E).(ii) que $\zeta \in f(\{\xi, \eta\})$. Como $\zeta \in D_{1} \subseteq D_{q}$, segue da definição de $q$ que $\zeta \in i_{q}(\{\xi, \eta\})$. Assim, para $\gamma=\zeta$ temos que $\zeta \in h_{q}(\gamma)$ e $\gamma \in i_{q}(\{\xi, \eta\})$.

Subcaso 4.2. $\zeta \in D_{2} \backslash D_{1}$.

Comecemos este subcaso provando o seguinte:

Observação 1. O conjunto $\left\{\delta_{\zeta}, \delta_{\xi}\right\}$ é não-vazio, $\min \left\{\delta_{\zeta}, \delta_{\xi}\right\}<\eta$ e, se $\delta_{\zeta}$ e $\delta_{\xi}$ existem, então $\delta_{\zeta} \neq \delta_{\xi}$.

Demonstração da Observação 1. Primeiramente, observe que, pela definição da operação $*$, temos que se $\xi \notin h_{q}(\eta)$, então $\zeta \in h_{q}(\xi) * h_{q}(\eta)=h_{q}(\xi) \cap h_{q}(\eta)$ e, portanto, $\zeta \in h_{q}(\eta)$. Analogamente, se $\xi \in h_{q}(\eta)$, então $\zeta \in h_{q}(\xi) * h_{q}(\eta)=h_{q}(\xi) \backslash h_{q}(\eta)$ e, portanto, $\zeta \notin h_{q}(\eta)$. 
Ou seja,

$$
\left|\{\zeta, \xi\} \cap h_{q}(\eta)\right|=1 .
$$

Daí, como neste subcaso temos que $\{\zeta, \xi\} \subseteq D_{2} \backslash D_{1}$ e $\eta \in D_{1} \backslash D_{2}$, segue da igualdade (II) que

$$
\left|\left\{\delta_{\zeta}, \delta_{\xi}\right\} \cap h_{1}(\eta)\right|=1
$$

e, portanto, o conjunto $\left\{\delta_{\zeta}, \delta_{\xi}\right\}$ é não-vazio.

Daí, observe que

$$
\min \left\{\delta_{\zeta}, \delta_{\xi}\right\} \leq \min \left(\left\{\delta_{\zeta}, \delta_{\xi}\right\} \cap h_{1}(\eta)\right) \leq \eta
$$

Como $\min \left\{\delta_{\zeta}, \delta_{\xi}\right\} \in D_{1} \cap D_{2}$, por definição e estamos no caso em que $\eta \notin D_{2}$, segue que $\min \left\{\delta_{\zeta}, \delta_{\xi}\right\}<\eta$.

Finalmente, como vimos que $\zeta \in h_{q}(\eta)$ se, e somente se, $\xi \notin h_{q}(\eta)$, então, se $\delta_{\zeta}$ e $\delta_{\xi}$ existem, segue que $\delta_{\zeta} \in h_{1}(\eta)$ se, e somente se, $\delta_{\xi} \notin h_{1}(\eta)$, de forma que $\delta_{\zeta} \neq \delta_{\xi}$, concluindo a demonstração da Observação 1.

Consideremos $\delta=\min \left\{\delta_{\xi}, \delta_{\eta}\right\}$ e observe que, como $\delta \in D_{1} \cap D_{2}$ e $\xi \notin D_{1}$, temos que $\delta \neq \xi$. Daí temos as seguintes opções:

Subcaso 4.2.1. $\delta<\xi$.

Aqui temos que $\delta \neq \delta_{\xi}$. Portanto, $\delta_{\zeta}$ existe e $\delta_{\zeta}=\delta \in D_{1} \cap \xi \cap \eta$. Por (E).(ii), temos que $\delta_{\zeta} \in f(\{\xi, \eta\}) \cap D_{q}=i_{q}(\{\xi, \eta\})$. Mas, pela definição de $\delta_{\zeta}, \zeta \in h_{2}\left(\delta_{\zeta}\right) \subseteq h_{q}\left(\delta_{\zeta}\right)$. Assim, para $\gamma=\delta_{\zeta}$, temos que $\zeta \in h_{q}(\gamma)$ e $\gamma \in i_{q}(\{\xi, \eta\})$, como queríamos.

Subcaso 4.2.2. $\delta>\xi$.

Neste caso, pela igualdade (III), como $\zeta \in h_{q}(\xi) * h_{q}(\eta) \subseteq h_{q}(\xi)$ e $\zeta, \xi \in D_{2} \backslash D_{1}$, temos que $\zeta \in h_{2}(\xi)$. Mostremos o seguinte:

Observação 2. $\zeta \in h_{2}(\xi) * h_{2}(\delta)$.

Demonstração da Observação 2. Primeiramente, suponhamos que $\delta=\delta_{\xi}$. Daí, se $\delta_{\zeta}$ também existe, então $\delta_{\xi}<\delta_{\zeta}$ e segue da minimalidade de $\delta_{\zeta}$ que $\zeta \notin h_{2}\left(\delta_{\xi}\right)$; é claro que se $\delta_{\zeta}$ não existe, então $\zeta \notin h_{2}\left(\delta_{\xi}\right)$. Mas pela definição de $\delta_{\xi}$, temos que $\xi \in h_{2}\left(\delta_{\xi}\right)$. Logo, 
$h_{2}(\xi) * h_{2}\left(\delta_{\xi}\right)=h_{2}(\xi) \backslash h_{2}\left(\delta_{\xi}\right)$ e, portanto, $\zeta \in h_{2}(\xi) \backslash h_{2}\left(\delta_{\xi}\right)=h_{2}(\xi) * h_{2}(\delta)$.

Agora, suponhamos que $\delta=\delta_{\zeta}$. Daí, se $\delta_{\xi}$ também existe, então $\delta_{\zeta}<\delta_{\xi}$ e segue da minimalidade de $\delta_{\xi}$ que $\xi \notin h_{2}\left(\delta_{\zeta}\right)$; é claro que se $\delta_{\xi}$ não existe, então $\xi \notin h_{2}\left(\delta_{\zeta}\right)$. Mas pela definição de $\delta_{\zeta}$, temos que $\zeta \in h_{2}\left(\delta_{\zeta}\right)$. Logo, $h_{2}(\xi) * h_{2}\left(\delta_{\zeta}\right)=h_{2}(\xi) \cap h_{2}\left(\delta_{\zeta}\right)$ e, portanto, $\zeta \in h_{2}(\xi) \cap h_{2}\left(\delta_{\zeta}\right)=h_{2}(\xi) * h_{2}(\delta)$, concluindo a demonstração da Observação 2.

Finalmente, como $p_{2} \in \mathbb{P}_{f}$, existe $\gamma \in i_{2}(\{\xi, \delta\})$ tal que $\zeta \in h_{2}(\gamma) \subseteq h_{q}(\gamma)$ e, por (E).(i), temos que:

$$
i_{2}(\{\xi, \delta\}) \subseteq f(\{\xi, \delta\}) \cap D_{2} \subseteq f(\{\xi, \eta\}) \cap D_{q}=i_{q}(\{\xi, \eta\}) .
$$

Logo, $\gamma \in i_{q}(\{\xi, \delta\})$ e $\zeta \in h_{q}(\gamma)$, concluindo a demonstração do Caso 4 , da Afirmação 4 e do fato que $q \in \mathbb{P}_{f}$.

Agora, segue facilmente da definição de $q$ e da Afirmação 1 que $q \leq p_{1}$. Analogamente, segue da definição de $q$ e da Afirmação 2 que $q \leq p_{2}$.

Por fim, verificamos que $q$ satisfaz a condição que queremos: sejam $\xi \in D_{1}$ e $\eta \in D_{2}$. Temos mais uma vez os seguintes casos:

Caso 1. $\xi \in D_{1} \cap D_{2}$.

Neste caso, (C) implica que $e(\xi)=\xi$. Daí, segue da igualdade (III) que $\xi \in h_{q}(\eta)$ se, e somente se, $e(\xi)=\xi \in h_{2}(\eta)$.

Caso 2. $\xi \in D_{1} \backslash D_{2}$.

Neste caso, segue diretamente da igualdade (IV) que $\xi \in h_{q}(\eta)$ se, e somente se $e(\xi) \in$ $h_{2}(\eta)$, e concluímos, desta forma, a demonstração do lema. 


\section{Capítulo 3}

\section{Um espaço de Asplund e suas propriedades topológicas}

Neste capítulo, vamos estudar as propriedades dos espaços $K_{1}$ e $C\left(K_{1}\right)$ :

Definição 3.1. Fixemos um modelo $V$ e sejam $\theta=\omega_{1}$ e $f:\left[\omega_{1}\right]^{2} \rightarrow\left[\omega_{1}\right] \leq \aleph_{0}$ dada por $f(\{\xi, \eta\})=\min \{\xi, \eta\}$. Fixemos um filtro $\mathbb{P}_{f}$-genérico $G$ sobre $V$. Vamos chamar de $L_{1} o$ espaço da Definição 2.7 correspondente ao forcing $\mathbb{P}_{f}$.

Observe que $f$ tem a propriedade $\Delta$ e, portanto, segue do Teorema 2.5 que $\mathbb{P}_{f}$ é c.c.c. e, portanto, preserva cardinais. Segue da Proposição 2.8 que $L_{1}$ é localmente compacto.

Notação 3.2. Vamos chamar de $K_{1}$ a compactificação de Alexandroff de $L_{1}$.

Segue dos Teoremas 2.10 e 2.11 que $L_{1}$ e $K_{1}$ são dispersos de peso $\aleph_{1}$ e, portanto, $C_{0}\left(L_{1}\right)$ e $C\left(K_{1}\right)$ são espaços de Asplund de densidade $\aleph_{1}$.

Vamos começar mostrando, na Seção 3.1, que (qualquer potência finita de) $K_{1}$ é hereditariamente separável. Para isso, vamos usar a amalgamação construída no Lema 2.13. Daí, segue que $C\left(K_{1}\right)$ é um espaço de Asplund de densidade $\aleph_{1}$ que não admite renormação Fréchet-diferenciável e não possui um sistema biortogonal não-enumerável.

Na Seção 3.2, mostramos uma proposição sobre a convergência na topologia fraca* de seqüências de $C\left(K_{1}\right)^{*}$, que é uma modificação do Lema 5.4 de Rabus [53]: aqui, o forcing $\mathbb{P}_{f}$ 
introduz uma topologia sobre $\omega_{1}$, enquanto que em [53], sobre $\omega_{2}$. Por outro lado, mostramos um resultado sobre convergência de medidas de Radon sobre o espaço $K_{1}$, enquanto que em [53] são considerados apenas pontos do espaço.

Finalmente, na Seção 3.3, usamos o resultado da seção anterior para dar um novo exemplo de um espaço que tem (C) e não tem (E). Podemos dizer, em linhas gerais, que a propriedade (C) é uma versão convexa da propriedade de Lindelöf e a propriedade (E), por sua vez, é uma versão convexa da seqüencialidade. A primeira foi introduzida por Corson [12] e a segunda, por Efremov [18]. O primeiro exemplo de um espaço que tem (C) e não tem (E) é uma modificação feita por Justin T. Moore (não publicada) do espaço de Ostaszewski [49] (assumindo, portanto, o princípio $\diamond$ ).

\subsection{Separabilidade hereditária}

Teorema 3.3. Em $V[G]$, para todo $n \in \mathbb{N}$, temos que $K_{1}^{n}$ é hereditariamente separável.

Demonstração. Vamos mostrar por indução em $n \in \mathbb{N}$ : fixemos $n \in \mathbb{N}$ e suponhamos que para todo $0 \leq i<n, K_{1}^{i}$ é hereditariamente separável (convencionamos $K_{1}^{0}=\{*\}=K_{1} \backslash L_{1}$ ). Mostremos que $K_{1}^{n}$ é hereditariamente separável. Para isso, pela Proposição 1.10, basta mostrar que $K_{1}^{n}$ não possui seqüências separadas à esquerda de cardinalidade $\aleph_{1}$.

Em $V$, suponha que $\left(\dot{x}_{\alpha}\right)_{\alpha<\omega_{1}}$ é uma seqüência de nomes tal que $\mathbb{P}_{f}$ força que $\left(\dot{x}_{\alpha}\right)_{\alpha<\omega_{1}}$ é uma seqüência de $K_{1}^{n}$, separada à esquerda e de cardinalidade $\aleph_{1}$ e que para cada $\alpha<\omega_{1}$, temos que $\dot{x}_{\alpha}=\left(\dot{x}_{1}^{\alpha}, \ldots, \dot{x}_{n}^{\alpha}\right)$, onde cada $\dot{x}_{i}^{\alpha}$ é um nome para um elemento de $K_{1}$.

Observe que se

$$
\mathbb{P}_{f} \Vdash \exists 1 \leq i \leq n, \exists X \subseteq \omega_{1},|X|=\aleph_{1} \text { tal que } \forall \alpha, \beta \in X, \dot{x}_{i}^{\alpha}=\dot{x}_{i}^{\beta},
$$

então

$$
\begin{gathered}
\mathbb{P}_{f} \Vdash \exists 1 \leq i \leq n, \exists X \subseteq \omega_{1},|X|=\aleph_{1} \text { tal que }\left(\left(\dot{x}_{1}^{\alpha}, \ldots, \dot{x}_{i-1}^{\alpha}, \dot{x}_{i+1}^{\alpha}, \ldots, \dot{x}_{n}^{\alpha}\right)\right)_{\alpha \in X} \\
\text { é uma seqüência separada à esquerda em } K_{1}^{n-1},
\end{gathered}
$$


contradizendo a hipótese indutiva. Portanto, podemos supor, sem perda de generalidade, que $\mathbb{P}_{f}$ força que para todo $1 \leq i \leq n$ e todo $\alpha<\beta<\omega_{1}, \dot{x}_{i}^{\alpha} \neq \dot{x}_{i}{ }^{\beta}$ e $\dot{x}_{i}^{\alpha} \in L_{1}=K_{1} \backslash\{*\}$.

Daí, para cada $\alpha<\omega_{1}$, existem $\dot{F}_{1}^{\alpha}, \ldots, \dot{F}_{n}^{\alpha}$ nomes para subconjuntos finitos de $\omega_{1}$ tais que $\mathbb{P}_{f}$ força que

$$
\forall \alpha<\omega_{1} \quad \forall 1 \leq i \leq n \quad \dot{x}_{i}^{\alpha} \in h\left(\dot{x}_{i}^{\alpha}\right) \backslash \bigcup_{\xi \in \dot{F}_{i}^{\alpha}} h(\xi)
$$

e

$$
\forall \alpha<\beta<\omega_{1} \quad \exists 1 \leq i \leq n \quad \dot{x}_{i}^{\alpha} \notin h\left(\dot{x}_{i}^{\beta}\right) \backslash \bigcup_{\xi \in \dot{F}_{i}^{\beta}} h(\xi)
$$

Para cada $\alpha<\omega_{1}$, sejam $p_{\alpha}=\left(D_{\alpha}, h_{\alpha}, i_{\alpha}\right) \in \mathbb{P}_{f}, x_{1}^{\alpha}, \ldots, x_{n}^{\alpha} \in \omega_{1}$ e $F_{1}^{\alpha}, \ldots, F_{n}^{\alpha} \subseteq \omega_{1}$ finitos tais que

$$
p_{\alpha} \Vdash \forall 1 \leq i \leq n \quad \dot{x}_{i}^{\alpha}=\check{x}_{i}^{\alpha} \text { e } \dot{F}_{i}^{\alpha}=\check{F}_{i}^{\alpha} .
$$

Pelo Lema 2.3, podemos assumir, sem perda de generalidade, que para todo $\alpha<\omega_{1}$ e todo $1 \leq i \leq n, F_{i}^{\alpha} \subseteq D_{\alpha}$ e $x_{i}^{\alpha} \in D_{\alpha}$. Pelo Lema 1.3, podemos também assumir que $\left(D_{\alpha}\right)_{\alpha<\omega_{1}}$ forma um $\Delta$-sistema de raiz $D$ tal que para todo $\alpha<\beta<\omega_{1}, D<D_{\alpha} \backslash D<D_{\beta} \backslash D$. Como, para cada $\xi \in D$ e cada $\alpha<\omega_{1}$, temos que $h_{\alpha}(\xi) \subseteq \xi \cap D_{\alpha} \subseteq D$, podemos supor que, para todo $\xi \in D$ e todo $\alpha<\beta<\omega_{1}, h_{\alpha}(\xi)=h_{\beta}(\xi)$. De forma análoga, para cada par $\{\xi, \eta\} \subseteq D$ e cada $\alpha<\omega_{1}$, temos que $i_{\alpha}(\{\xi, \eta\}) \in[f(\{\xi, \eta\})]^{<\aleph_{0}}$ e podemos supor então que, para todo par $\{\xi, \eta\} \subseteq D$ e todo $\alpha<\beta<\omega_{1}, i_{\alpha}(\{\xi, \eta\})=i_{\beta}(\{\xi, \eta\})$. Finalmente, observe que podemos supor que, para todo $1 \leq i \leq n$, tem-se que: ou $x_{i}^{\alpha}=x_{i}^{\beta}$ para todo $\alpha<\beta<\omega_{1}$, ou $x_{i}^{\alpha} \notin D$ para todo $\alpha<\omega_{1}$. Além disso, suponha, sem perda de generalidade, que para todo $1 \leq i<j \leq n$ e todo $\alpha<\beta<\omega_{1}, x_{i}^{\alpha}=x_{j}^{\alpha}$ se, e somente se $x_{i}^{\beta}=x_{j}^{\beta}$.

Sejam $\alpha<\beta<\omega_{1}$ e observe que $p_{\alpha}, p_{\beta},\left\{x_{1}^{\alpha}, \ldots, x_{n}^{\alpha}\right\} \backslash D$ e $\left\{x_{1}^{\beta}, \ldots, x_{n}^{\beta}\right\} \backslash D$ satisfazem as condições (A), (B), (C) e (D) do Lema 2.13. Logo, existe $q \leq p_{\alpha}, p_{\beta}$ em $\mathbb{P}_{f}$ tal que, para todo $\xi \in D_{\beta}$ e todo $1 \leq i \leq n$, se $x_{i}^{\alpha} \notin D$, então

$$
x_{i}^{\alpha} \in h_{q}(\xi) \text { se, e somente se, } x_{i}^{\beta} \in h_{q}(\xi) .
$$

Como $q \leq p_{1}, p_{2}$, temos que

$$
q \Vdash \forall 1 \leq i \leq n \quad \dot{x}_{i}^{\alpha}=\check{x}_{i}^{\alpha}, \dot{x}_{i}^{\beta}=\check{x}_{i}^{\beta}, \dot{F}_{i}^{\alpha}=\check{F}_{i}^{\alpha}, \dot{F}_{i}^{\beta}=\check{F}_{i}^{\beta} .
$$


Mas, para cada $1 \leq i \leq n$, se $x_{i}^{\alpha} \in D$, então $x_{i}^{\beta}=x_{i}^{\alpha}$ e daí,

$$
q \Vdash \dot{x}_{i}^{\alpha}=\check{x}_{i}^{\alpha}=\check{x}_{i}^{\beta}=\dot{x}_{i}^{\beta},
$$

contradizendo o fato que assumimos que $\mathbb{P}_{f}$ força que $\dot{x}_{i}^{\alpha}=\dot{x}_{i}^{\beta}$. Logo, para todo $1 \leq i \leq n$, $x_{i}^{\alpha} \notin D$. Como

$$
\forall 1 \leq i \leq n, x_{i}^{\beta} \in h_{\beta}\left(x_{i}^{\beta}\right) \backslash \bigcup_{\xi \in F_{i}^{\beta}} h_{\beta}(\xi)
$$

e $F_{i}^{\beta} \cup\left\{x_{i}^{\beta}\right\} \subseteq D_{\beta}$, segue que

$$
\forall 1 \leq i \leq n, x_{i}^{\alpha} \in h_{\beta}\left(x_{i}^{\beta}\right) \backslash \bigcup_{\xi \in F_{i}^{\beta}} h_{\beta}(\xi),
$$

ou seja,

$$
q \Vdash \forall 1 \leq i \leq n \quad \dot{x}_{i}^{\alpha}=\check{x}_{i}^{\alpha} \in h\left(\check{x}_{i}^{\beta}\right) \backslash \bigcup_{\xi \in \check{F}_{i}^{\beta}} h(\xi)=h\left(\dot{x}_{i}^{\beta}\right) \backslash \bigcup_{\xi \in \dot{F}_{i}^{\beta}} h(\xi),
$$

contradizendo a hipótese e concluindo a demonstração.

Para terminar esta seção, temos as seguintes conseqüências sobre o espaço de Banach $C\left(K_{1}\right)$ :

Teorema 3.4. Em $V[G], C\left(K_{1}\right)$ não admite renormação Fréchet-diferenciável e não contém nenhum sistema biortogonal não-enumerável.

Demonstração. Segue do Teorema 3.3 que $h d\left(K_{1}^{n}\right)=\aleph_{0}$ para todo $n \in \mathbb{N}$. Por outro lado, pelo Teorema 2.11, temos que $w\left(K_{1}\right)=\aleph_{1}$. Daí, segue do Corolário 1.45 que $C\left(K_{1}\right)$ não admite renormação Fréchet-diferenciável e não contém nenhum sistema biortogonal nãoenumerável.

\subsection{Convergência de seqüências na topologia fraca*}

Vejamos agora um resultado sobre a convergência de seqüências de $C\left(K_{1}\right)^{*}$ com respeito à topologia fraca*. Observe que segue do resultado abaixo que, em particular, não existe uma seqüência de pontos de $L_{1}$ que converge para $*$ em $K_{1}$. 
Proposição 3.5. Em $V[G]$, se $\left(\mu_{n}\right)_{n \in \mathbb{N}} \subseteq B_{C\left(K_{1}\right)^{*}}$ é uma seqüência que converge para $\delta_{*}$ com respeito à topologia fraca* ${ }^{*}$, então existe $n_{0} \in \mathbb{N}$ tal que para todo $n \in \mathbb{N}, n \geq n_{0}$, tem-se que $\mu_{n}(\{*\}) \neq 0$.

Demonstração. Senão, existe, em $V[G]$, uma seqüência $\left(\mu_{n}\right)_{n \in \mathbb{N}} \subseteq B_{C\left(K_{1}\right)^{*}}$ que converge para $\delta_{*}$ com respeito à topologia fraca* e tal que para todo $n \in \mathbb{N}$, tem-se que $\mu_{n}(\{*\})=0$.

Em $V$, fixe $0<\varepsilon<\frac{1}{5}$ e sejam $\dot{\delta}_{*}$ um nome para $\delta_{*}$ e $\left(\dot{\mu}_{n}\right)_{n \in \mathbb{N}}$ uma seqüência de nomes para elementos de $B_{C\left(K_{1}\right)^{*}}$, tais que

$\mathbb{P}_{f} \Vdash \forall n \in \mathbb{N}, \dot{\mu}_{n}(\{*\})=0$ e $\left(\dot{\mu}_{n}\right)_{n \in \mathbb{N}}$ converge para $\dot{\delta}_{*}$ com respeito à topologia fraca*.

Daí, segue do Teorema 1.27 que

$$
\mathbb{P}_{f} \Vdash \forall n \in \mathbb{N}, \exists F_{n} \subseteq K_{1} \backslash\{*\}=L_{1} \text { tal que }\left|\dot{\mu}_{n}\right|\left(K_{1} \backslash F_{n}\right)<\check{\varepsilon} .
$$

Assim, para cada $n \in \mathbb{N}$, seja $A_{n}$ uma anticadeia maximal em $\mathbb{P}_{f}$ tal que para cada $p \in A_{n}$, existe um subconjunto finito $F_{n}^{p}$ de $\omega_{1}$ tal que $p$ força que $\left|\dot{\mu}_{n}\right|\left(K_{1} \backslash \check{F}_{n}^{p}\right)<\check{\varepsilon}$ e para cada $\alpha \in F_{n}^{p}$, existe $a_{\alpha} \in \mathbb{R}$ tal que $p$ força que $\dot{\mu}_{n}(\{\check{\alpha}\})=\check{a}_{\check{\alpha}}$. Pelo Lema 2.3, podemos assumir, sem perda de generalidade, que para todo $n \in \mathbb{N}$ e todo $p \in A_{n}, F_{n}^{p} \subseteq D_{p}$.

Do fato que $\mathbb{P}_{f}$ é c.c.c., segue que existe $\gamma<\omega_{1}$ tal que

$$
\bigcup\left\{D_{p}: p \in A_{n}, n \in \mathbb{N}\right\} \subseteq \gamma
$$

Seja $q \in \mathbb{P}_{f}$. Observe que $h_{q}(\gamma) \subseteq \gamma \subseteq \omega_{1}$ e, portanto, $q \Vdash * \notin h(\gamma)$. Daí, $q \Vdash \dot{\delta}_{*}(h(\gamma))=$ 0 . Como $\mathbb{P}_{f}$ força que $\left(\dot{\mu}_{n}\right)_{n \in \mathbb{N}}$ converge para $\dot{\delta}_{*}$ com respeito à topologia fraca*, existem $r \leq q$ e $m \in \mathbb{N}$ tais que

$$
r \Vdash \forall n \geq m,\left|\dot{\mu}_{n}(h(\gamma))\right|<\check{\varepsilon} .
$$

Mais uma vez pelo Lema 2.3, podemos assumir, sem perda de generalidade, que $\gamma \in D_{r}$. Seja $H=D_{r} \cap \gamma$ e $E=D_{r} \backslash \gamma=\left\{\gamma=\alpha_{1}<\cdots<\alpha_{k}\right\}$. Seja $F \subseteq \omega_{1}$, tal que $E<F$ e $|F|=2|E|$ e denote $F=\left\{\alpha_{i}^{1}, \alpha_{i}^{2}: 1 \leq i \leq k\right\}$. 
Vamos obter, após 3 passos, $u \in \mathbb{P}_{f}$ e $n \in \mathbb{N}$ tais que $u \leq r, n \geq m$ e $u \Vdash\left|\dot{\mu}_{n}(h(\gamma))\right|>\check{\varepsilon}$, contradizendo a hipótese: no Passo 1, estendemos $r$ a uma condição $s$ tal que $D_{s}=D_{r} \cup F$ e para todo $1 \leq i \leq k$, temos que $h_{s}\left(\alpha_{i}^{1}\right) \cap h_{s}\left(\alpha_{i}^{2}\right)=\bigcup_{\xi \in D_{r}} h_{s}(\xi)$; no Passo 2, estendemos $s$ a uma condição $t$ tal que $D_{t} \subseteq \gamma \cup E \cup F$ de forma que existe $n \geq m$ e $p \in A_{n}$ tal que $t \leq p$ e

$$
t \Vdash\left|\dot{\mu}_{n}\left(\bigcup_{\xi \in \check{D}_{r}} h(\xi)\right)\right|<\check{\varepsilon} ;
$$

finalmente, no Passo 3 vamos obter $u$ tal que $D_{u}=\left(D_{t} \cap \gamma\right) \cup E, u \leq r$ e

$$
u \Vdash\left|\dot{\mu}_{n}(h(\gamma))\right|>\check{\varepsilon},
$$

contradizendo a hipótese.

Passo 1. Defina $s=\left(D_{s}, h_{s}, i_{s}\right)$ por $D_{s}=D_{r} \cup F$;

$$
h_{s}(\xi)= \begin{cases}h_{r}(\xi) & \text { se } \xi \in D_{r} \\ D_{r} \cup\{\xi\} & \text { se } \xi \in F\end{cases}
$$

$\mathrm{e}$

$$
i_{s}(\{\xi, \eta\})= \begin{cases}i_{r}(\{\xi, \eta\}) & \text { se } \xi, \eta \in D_{r}, \\ \min \{\xi, \eta\} \cap D_{s} & \text { caso contrário. }\end{cases}
$$

É evidente que $s$ satisfaz as condições 1 e 2 da Definição 2.1 e as condições 3.(a) e (b) para $\xi, \eta \in D_{r}$ seguem do fato que $r \in \mathbb{P}_{f}$.

Sejam então $\xi \in D_{r}$ e $\eta \in F$. Daí, $\xi \in h_{s}(\eta)$ e $h_{s}(\xi) \subseteq h_{s}(\eta)$ de forma que $h_{s}(\xi) * h_{s}(\eta)=$ $h_{s}(\xi) \backslash h_{s}(\eta)=\emptyset$. Logo, $s$ satisfaz as condições 3.(a) e (b) da Definição 2.1 para esses pares.

Sejam agora $\xi, \eta \in F$ e $\xi<\eta$. Neste caso, $\xi \notin h_{s}(\eta)$ e daí, $h_{s}(\xi) * h_{s}(\eta)=h_{s}(\xi) \cap h_{s}(\eta)=$ $D_{r}$. Mas $D_{r}=H \cup E \subseteq \xi \cap D_{s}$, de forma que $s$ satisfaz as condições 3.(a) e 3.(b) para esses pares.

Portanto, $s \in \mathbb{P}_{f}$ e é fácil ver que $s \leq r$.

Passo 2. Observe que $\mathbb{P}_{f}$ força que $\bigcup_{\xi \in \check{D}_{r}} h(\xi)$ é um aberto-fechado e $* \notin \bigcup_{\xi \in \check{D_{r}}} h(\xi)$. Daí, 
temos por hipótese que $\mathbb{P}_{f}$ força que $\dot{\delta}_{*}\left(K_{1} \backslash \bigcup_{\xi \in \check{D}_{r}} h(\xi)\right)=1$.

Como $\mathbb{P}_{f}$ força que $\left(\dot{\mu}_{n}\right)_{n \in \mathbb{N}}$ converge para $\dot{\delta}_{*}$ com respeito à topologia fraca*, existem $t \leq s$ e $n \in \mathbb{N}, n \geq m$ tais que

$$
t \Vdash \dot{\mu}_{n}\left(K_{1} \backslash \bigcup_{\xi \in \check{D}_{r}} h(\xi)\right)>1-\check{\varepsilon} .
$$

Mas $A_{n}$ é uma anticadeia maximal e daí, podemos assumir, sem perda de generalidade, que existe $p \in A_{n}$ tal que $t \leq p$. Como $t \leq p, r$, temos que

$$
t \Vdash \dot{\mu}_{n}\left(\check{F}_{n}^{p} \backslash \bigcup_{\xi \in \check{D}_{r}} h(\xi)\right) \geq \dot{\mu}_{n}\left(K_{1} \backslash \bigcup_{\xi \in \check{D}_{r}} h(\xi)\right)-\left|\dot{\mu}_{n}\right|\left(K_{1} \backslash \check{F}_{n}^{p}\right)>1-2 \check{\varepsilon}
$$

ou seja,

$$
\sum\left\{a_{\alpha}: \alpha \in F_{n}^{p} \backslash \bigcup_{\xi \in D_{r}} h_{t}(\xi)\right\}>1-2 \varepsilon
$$

Passo 3. Seja $T=D_{t} \cap \gamma$ e observe que $t, T, E, F$ e $H$ satisfazem as hipóteses do Lema 2.12. Logo, existe $u=\left(D_{u}, h_{u}, i_{u}\right) \in \mathbb{P}_{f}$ tal que $D_{u}=T \cup E, u \leq\left. t\right|_{T}, u \leq\left. t\right|_{H \cup E}$ e $T \backslash \bigcup_{\xi \in H \cup E} h_{t}(\xi) \subseteq h_{u}\left(\alpha_{1}\right)$ e observe que $\left.t\right|_{T} \leq p$, que $H \cup E=D_{r}$ e que $\left.t\right|_{H \cup E}=r$.

Resta mostrar a afirmação abaixo e temos uma contradição com o fato que $u \leq r$ e que $r \Vdash\left|\dot{\mu}_{n}(h(\gamma))\right|<\check{\varepsilon}:$

Afirmação. $u \Vdash \dot{\mu}_{n}(h(\gamma))>\check{\varepsilon}$.

Demonstração da Afirmação. Considere

$$
I=\left\{\alpha \in F_{n}^{p}: t \Vdash \check{\alpha} \notin \bigcup_{\xi \in \check{D}_{r}} h(\xi)\right\}=F_{n}^{p} \backslash \bigcup_{\xi \in D_{r}} h_{t}(\xi)
$$

e note que, como $D_{r}=H \cup E, \alpha_{1}=\gamma$ e $F_{n}^{p} \subseteq D_{p} \subseteq D_{t} \cap \gamma=T$, temos que

$$
I \subseteq T \backslash \bigcup_{\xi \in D_{r}} h_{t}(\xi) \subseteq h_{u}(\gamma)
$$


Como $u \leq\left. t\right|_{T} \leq p$ e $p$ força que $\dot{\mu}_{n}(\{\check{\alpha}\})=\check{a}_{\check{\alpha}}$ para todo $\alpha \in F_{n}^{p}$, temos que

$$
u \Vdash \dot{\mu}_{n}(\check{I})=\sum_{\alpha \in \check{I}} \check{a}_{\alpha}>1-2 \check{\varepsilon},
$$

e, como $\mathbb{P}_{f}$ força que $\left\|\dot{\mu}_{n}\right\| \leq 1$, temos que

$$
u \Vdash\left|\dot{\mu}_{n}\right|(h(\gamma) \backslash \check{I}) \leq\left|\dot{\mu}_{n}\right|\left(K_{1} \backslash \check{I}\right) \leq\left\|\dot{\mu}_{n}\right\|-\left|\dot{\mu}_{n}\right|(\check{I})<1-(1-2 \check{\varepsilon})=2 \check{\varepsilon} .
$$

Portanto,

$$
u \Vdash \dot{\mu}_{n}(h(\gamma)) \geq \dot{\mu}_{n}(\check{I})-\left|\dot{\mu}_{n}\right|(h(\gamma) \backslash \check{I})>1-4 \check{\varepsilon}>\check{\varepsilon},
$$

concluindo a demonstração da afirmação e da proposição.

\subsection{A propriedade (C) e a propriedade (E)}

Nesta seção, vamos considerar duas versões convexas de propriedades topológicas. A primeira delas é uma versão da propriedade de Lindelöf:

Definição 3.6 (Corson [12]). Seja X um espaço de Banach. Dizemos que X tem a propriedade (C) se toda família de subconjuntos fechados e convexos cuja intersecção é vazia possui uma subfamília enumerável de intersecção vazia.

Proposição 3.7. Se X é um espaço de Banach fracamente Lindelöf, então X tem a propriedade $(C)$.

Demonstração. Seja $\mathcal{F}$ uma família de subconjuntos fechados e convexos cuja intersecção é vazia. Pelo Teorema 1.28, temos que todo elemento de $\mathcal{F}$ é fracamente fechado. Assim, $\mathscr{C}=\{X \backslash F: F \in \mathcal{F}\}$ é uma família de subconjuntos de $X$ fracamente abertos que recobrem $X$. Como $X$ é fracamente Lindelöf, existe um subrecobrimento enumerável $\mathscr{C}^{*}$ de $\mathscr{C}$. Segue que $\mathcal{F}^{*}=\left\{F \in \mathcal{F}: X \backslash F \in \mathscr{C}^{*}\right\}$ é uma subfamília enumerável de $\mathcal{F}$ de intersecção vazia.

Passemos então à segunda propriedade, esta, uma versão convexa da seqüencialidade do espaço dual: 
Definição 3.8 (Efremov [18]). Seja $X$ um espaço de Banach. Dizemos que $X$ tem a propriedade (E) quando para todo conjunto limitado e convexo $C$ de $X^{*}$, se $\varphi \in \bar{C} w^{w^{*}}$, então existe uma seqüência $\left(\varphi_{n}\right)_{n \in \mathbb{N}} \subseteq C$ tal que $\left(\varphi_{n}\right)_{n \in \mathbb{N}}$ converge para $\varphi$ com respeito à topologia fraca*.

Plichko e Yost ([51], pág. 352) perguntaram se (C) implica (E) e Justin T. Moore obteve uma modificação do espaço de Ostaszewski, usando o princípio conjuntístico $\diamond$, que tem (C) e não tem (E). O espaço que apresentamos neste capítulo é também um contra-exemplo para a conjectura de Plichko e Yost:

Teorema 3.9. Em $V[G], C\left(K_{1}\right)$ tem a propriedade $(C)$ e não tem a propriedade $(E)$.

Demonstração. Pelos Teoremas 1.44 e 3.3 temos que $C\left(K_{1}\right)$ é fracamente Lindelöf e, pela Proposição 3.7 , segue que $C\left(K_{1}\right)$ tem a propriedade $(\mathrm{C})$.

Para mostrar que, em $V[G], C\left(K_{1}\right)$ não tem a propriedade (E), observe que o conjunto

$$
C=\left\{\sum_{i=1}^{k} a_{i} \delta_{x_{i}}: k \in \mathbb{N}, a_{i} \in(0,1], x_{i} \in L_{1}=K_{1} \backslash\{*\}, \sum_{i=1}^{k} a_{i}=1\right\},
$$

é um subconjunto convexo de $S_{C\left(K_{1}\right)^{*}}$.

Vejamos que $\delta_{*} \in \bar{C}^{w^{*}}$. Dados $\varepsilon>0, f_{1}, \ldots, f_{n} \in C\left(K_{1}\right)$, temos que

$$
U=\bigcap_{i=1}^{n} f_{i}^{-1}\left[\left(f_{i}(*)-\varepsilon, f_{i}(*)+\varepsilon\right)\right]
$$

é uma vizinhança aberta de $*$. Como $*$ é um ponto de acumulação de $K_{1}$, existe $x \in L_{1} \cap U$ e segue que

$$
\delta_{x} \in\left\{\mu \in C\left(K_{1}\right)^{*}: \forall 1 \leq i \leq n,\left|\mu\left(f_{i}\right)-\delta_{*}\left(f_{i}\right)\right|<\varepsilon\right\} \cap C .
$$

Logo, $\delta_{*} \in \bar{C}^{w^{*}}$.

Por outro lado, pela Proposição 3.5, não existe uma seqüência em $C$ que converge para $\delta_{*}$ com respeito à topologia fraca*. Portanto, $C\left(K_{1}\right)$ não possui a propriedade $(\mathrm{E})$. 


\section{Capítulo 4}

\section{Um espaço de Asplund sem sistema biortogonal não-enumerável}

Neste capítulo, vamos estudar as propriedades dos espaços $K_{2}$ e $C\left(K_{2}\right)$ :

Definição 4.1. Fixemos um modelo $V$, seja $\theta=\omega_{2}$ e suponhamos que existe $f:\left[\omega_{2}\right]^{2} \rightarrow$ $\left[\omega_{2}\right]^{\leq \aleph_{0}}$ uma função $o^{1}$ tal que para todo $\xi<\eta<\omega_{2}, f(\{\xi, \eta\}) \subseteq \xi$ e se A é uma família nãoenumerável de subconjuntos finitos de $\omega_{2}$ que forma um $\Delta$-sistema, então existem $a, b \in A$ e uma bijeção e $: a \rightarrow b$ que preserva ordem, constante em $a \cap b$ e tal que para todo $\xi \in a$, $\xi \leq e(\xi)$ e para todo $\zeta \in a \cap b$, todo $\xi \in a \backslash b$ e todo $\eta \in b \backslash a$ :

(i) se $\zeta<\xi$, então $f(\{\zeta, \eta\}) \subseteq f(\{\xi, \eta\})$;

(ii) se $\zeta<\eta$, então $f(\{\zeta, \xi\}) \subseteq f(\{\xi, \eta\})$;

(iii) $a \cap \xi \cap \eta \subseteq f(\{\xi, \eta\})$.

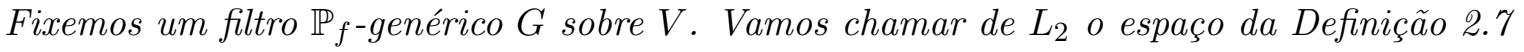
correspondente ao forcing $\mathbb{P}_{f}$.

\footnotetext{
${ }^{1}$ Observe que o Teorema A.22 garante a existência de um forcing que força a existência de uma função $f$ com as propriedades aqui enunciadas, garantindo, portanto, que a existência dessa função é consistente com ZFC. Logo, podemos assumir que essa função existe no modelo inicial $V$.
} 
Observe que $f$ tem a propriedade $\Delta$ e, portanto, segue do Teorema 2.5 que $\mathbb{P}_{f}$ é c.c.c. e, portanto, preserva cardinais. Segue da Proposição 2.8 que $L_{2}$ é localmente compacto.

Notação 4.2. Vamos chamar de $K_{2}$ a compactificação de Alexandroff de $L_{2}$.

Segue dos Teoremas 2.10 e 2.11 que $L_{2}$ e $K_{2}$ são dispersos de peso $\aleph_{2}$ e, portanto, $C_{0}\left(L_{2}\right)$ e $C\left(K_{2}\right)$ são espaços de Asplund de densidade $\aleph_{2}$.

Começamos, na Seção 4.1, aplicando o Lema 2.14 para mostrar que toda potência finita do espaço $K_{2}$ é hereditariamente separável. Para isso, usamos fortemente as propriedades satisfeitas pela função $f$ da Definição 4.1, lembrando que estas são mais fortes que a propriedade $\Delta$ e que as satisfeitas pela função construída em [5].

Na seção seguinte, 4.2, vamos analisar outras propriedades interessantes do espaço $K_{2} \mathrm{e}$ suas conseqüências para o espaço de Banach $C\left(K_{2}\right)$.

Primeiramente, mostramos que $K_{2}$ tem altura $\omega_{2}$ e é, portanto, o primeiro exemplo consistente de um espaço compacto e disperso, hereditariamente separável de altura $\omega_{2}$. Lembramos que o primeiro exemplo consistente de um espaço compacto e disperso, de largura enumerável e altura $\omega_{2}$ é o de Baumgartner e Shelah [5]. Por outro lado, a hipótese do contínuo implica que não existem espaços como o de [5] ou como $K_{2}$ e Just [35] mostrou, usando o forcing de Cohen, que a não existência destes espaços também é consistente com a negação da hipótese do contínuo.

Ainda do ponto de vista topológico, temos que o grau de Lindelöf hereditário de $K_{2}$ é $\aleph_{2}$, de forma que nosso espaço é o primeiro contra-exemplo compacto consistente para a desigualdade $h L(K) \leq h d(K)^{+}$. Lembramos que a desigualdade dual para espaços compactos segue de um resultado de Shapirovskiı̌ [62], ou seja, vale em ZFC que para todo espaço compacto $K, h d(K) \leq h L(K)^{+}$.

Passamos finalmente às propriedades do espaço de Banach $C\left(K_{2}\right)$. Assim como no capítulo anterior, segue do fato que todas as potências finitas de $K_{2}$ são hereditariamente separáveis, que $C\left(K_{2}\right)$ não admite renormação Fréchet-diferenciável e não contém nenhum sistema biortogonal não-enumerável. Além disso, a densidade de $C\left(K_{2}\right)$ é $\aleph_{2}$, assim como o peso de $K_{2}$. Temos, portanto, o primeiro exemplo consistente de um espaço de Banach $C(K)$ de densidade estritamente maior que $\aleph_{1}$ sem nenhum sistema biortogonal não-enumerável. Todorcevic [65] mostrou que todo espaço de Banach da forma $C(K)$ de densidade estrita- 
mente maior que $\aleph_{1}$ possui um sistema semi-biortogonal não-enumerável. Assim, segue do nosso exemplo que não se pode generalizar o resultado de Todorcevic em ZFC, substituindose a existência de um sistema semi-biortogonal não-enumerável pela existência de um sistema biortogonal não-enumerável.

\subsection{Separabilidade hereditária}

Teorema 4.3. Em $V[G]$, para todo $n \in \mathbb{N}$, temos que $K_{2}^{n}$ é hereditariamente separável.

Demonstração. Vamos mostrar por indução em $n \in \mathbb{N}$ : fixemos $n \in \mathbb{N}$ e suponhamos que para todo $0 \leq i<n, K_{2}^{i}$ é hereditariamente separável (convencionamos $K_{2}^{0}=\{*\}$ ). Mostremos que $K_{2}^{n}$ é hereditariamente separável. Para isso, pela Proposição 1.10, basta mostrar que $K_{2}^{n}$ não possui seqüências separadas à esquerda de cardinalidade $\aleph_{1}$.

Em $V$, suponha que $\left(\dot{x}_{\alpha}\right)_{\alpha<\omega_{1}}$ é uma seqüência de nomes tal que $\mathbb{P}_{f}$ força que $\left(\dot{x}_{\alpha}\right)_{\alpha<\omega_{1}}$ é uma seqüência de $K_{2}^{n}$, separada à esquerda e de cardinalidade $\aleph_{1}$ e que para cada $\alpha<\omega_{1}$, temos que $\dot{x}_{\alpha}=\left(\dot{x}_{1}^{\alpha}, \ldots, \dot{x}_{n}^{\alpha}\right)$, onde cada $\dot{x}_{i}^{\alpha}$ é um nome para um elemento de $K_{2}$.

Observe que se

$$
\mathbb{P}_{f} \Vdash \exists 1 \leq i \leq n, \exists X \subseteq \omega_{1},|X|=\aleph_{1} \text { tal que } \forall \alpha, \beta \in X, \dot{x}_{i}^{\alpha}=\dot{x}_{i}^{\beta},
$$

então

$$
\begin{gathered}
\mathbb{P}_{f} \Vdash \exists 1 \leq i \leq n, \exists X \subseteq \omega_{1},|X|=\aleph_{1} \text { tal que }\left(\left(\dot{x}_{1}^{\alpha}, \ldots, \dot{x}_{i-1}^{\alpha}, \dot{x}_{i+1}^{\alpha}, \ldots, \dot{x}_{n}^{\alpha}\right)\right)_{\alpha \in X} \\
\text { é uma seqüência separada à esquerda em } K_{2}^{n-1},
\end{gathered}
$$

contradizendo a hipótese indutiva. Portanto, podemos supor, sem perda de generalidade, que $\mathbb{P}_{f}$ força que para todo $1 \leq i \leq n$ e todo $\alpha<\beta<\omega_{1}, \dot{x}_{i}^{\alpha} \neq \dot{x}_{i}{ }^{\beta}$ e $\dot{x}_{i}^{\alpha} \in L_{2}=K_{2} \backslash\{*\}$.

Daí, para cada $\alpha<\omega_{1}$, existem $\dot{F}_{1}^{\alpha}, \ldots, \dot{F}_{n}^{\alpha}$ nomes para subconjuntos finitos de $\omega_{2}$ tais que $\mathbb{P}_{f}$ força que

$$
\forall \alpha<\omega_{1} \quad \forall 1 \leq i \leq n \quad \dot{x}_{i}^{\alpha} \in h\left(\dot{x}_{i}^{\alpha}\right) \backslash \bigcup_{\xi \in \dot{F}_{i}^{\alpha}} h(\xi)
$$


e

$$
\forall \alpha<\beta<\omega_{1} \quad \exists 1 \leq i \leq n \quad \dot{x}_{i}^{\alpha} \notin h\left(\dot{x}_{i}^{\beta}\right) \backslash \bigcup_{\xi \in \dot{F}_{i}^{\beta}} h(\xi)
$$

Para cada $\alpha<\omega_{1}$, sejam $p_{\alpha}=\left(D_{\alpha}, h_{\alpha}, i_{\alpha}\right) \in \mathbb{P}_{f}, x_{1}^{\alpha}, \ldots, x_{n}^{\alpha} \in \omega_{2}$ e $F_{1}^{\alpha}, \ldots, F_{n}^{\alpha} \subseteq \omega_{2}$ finitos tais que

$$
p_{\alpha} \Vdash \forall 1 \leq i \leq n \quad \dot{x}_{i}^{\alpha}=\check{x}_{i}^{\alpha} \text { e } \dot{F}_{i}^{\alpha}=\check{F}_{i}^{\alpha} .
$$

Pelo Lema 2.3, podemos assumir, sem perda de generalidade, que para todo $\alpha<\omega_{1}$ e todo $1 \leq i \leq n, F_{i}^{\alpha} \subseteq D_{\alpha}$ e $x_{i}^{\alpha} \in D_{\alpha}$.

Pelo Lema 1.3, podemos também assumir que $\left(D_{\alpha}\right)_{\alpha<\omega_{1}}$ forma um $\Delta$-sistema de raiz $D$. Como, para cada par $\{\xi, \eta\} \subseteq D$ e cada $\alpha<\omega_{1}$, temos que $i_{\alpha}(\{\xi, \eta\}) \in[f(\{\xi, \eta\})]^{<\aleph_{0}}$, podemos supor ainda que para todo $\alpha<\beta<\omega_{1}$, se $\xi, \eta \in D, \xi \neq \eta$, então $i_{\alpha}(\{\xi, \eta\})=$ $i_{\beta}(\{\xi, \eta\})$.

Daí, podemos assumir também, sem perda de generalidade, que $\left(D_{\alpha}\right)_{\alpha<\omega_{1}}$ forma um $\Delta$-sistema de raiz $D$ tal que para cada par $\alpha<\beta<\omega_{1}$, existe uma função $e_{\alpha \beta}: D_{\alpha} \rightarrow D_{\beta}$ bijetora que preserva ordem e tal que:

- se $\xi, \eta \in D_{\alpha}$, então $\xi \in h_{\alpha}(\eta)$ se, e somente se, $e_{\alpha \beta}(\xi) \in h_{\beta}\left(e_{\alpha \beta}(\eta)\right)$;

- se $\xi \in D$, então $e_{\alpha \beta}(\xi)=\xi$;

- se $\xi \in D_{\alpha}$, então $\xi \leq e_{\alpha \beta}(\xi)$;

- para todo $1 \leq i \leq n, e_{\alpha \beta}\left(x_{i}^{\alpha}\right)=x_{i}^{\beta}$.

Daí, podemos supor que para todo $1 \leq i \leq n$, temos que: ou $x_{i}^{\alpha}=x_{i}^{\beta}$ para todo $\alpha<\beta<\omega_{1}$; ou $x_{i}^{\alpha} \notin D$ para todo $\alpha<\omega_{1}$.

Finalmente, pelas propridades da função $f$ da Definição 4.1, existem $\alpha<\beta<\omega_{1}$ tais que para todo $\zeta \in D$, todo $\xi \in D_{\alpha} \backslash D$ e todo $\eta \in D_{\beta} \backslash D$ :

(i) se $\zeta<\xi$, então $f(\{\zeta, \eta\}) \subseteq f(\{\xi, \eta\})$;

(ii) se $\zeta<\eta$, então $f(\{\zeta, \xi\}) \subseteq f(\{\xi, \eta\})$; 
(iii) $D_{\alpha} \cap \xi \cap \eta \subseteq f(\{\xi, \eta\})$.

Temos assim que $p_{\alpha}$ e $p_{\beta}$ satisfazem todas as hipóteses do Lema 2.14. Daí, existe $q \leq p_{\alpha}, p_{\beta}$ em $\mathbb{P}_{f}$ tal que para todo $\xi \in D_{\alpha}$ e todo $\eta \in D_{\beta}$,

$$
\xi \in h_{q}(\eta) \text { se, e somente se, } e_{\alpha \beta}(\xi) \in h_{2}(\eta) \text {. }
$$

Daí, para todo $1 \leq i \leq n$ e todo $\xi \in D_{\beta}$, temos que

$$
x_{i}^{\alpha} \in h_{q}(\xi) \text { se, e somente se, } x_{i}^{\beta} \in h_{2}(\xi) .
$$

Assim, temos que

$$
x_{i}^{\alpha} \in h_{q}\left(x_{i}^{\beta}\right) \backslash \bigcup_{\xi \in F_{i}^{\beta}} h_{q}(\xi) .
$$

Mas $q \leq p_{\alpha}, p_{\beta}$ e então

$$
q \Vdash \forall 1 \leq i \leq n, \dot{x}_{i}^{\alpha}=\check{x}_{i}^{\alpha}, \dot{x}_{i}^{\beta}=\check{x}_{i}^{\beta} \text { e } \dot{F}_{i}^{\beta}=\check{F}_{i}^{\beta}
$$

Portanto,

$$
q \Vdash \forall 1 \leq i \leq n, \dot{x}_{i}^{\alpha}=\check{x}_{i}^{\alpha} \in h\left(\check{x}_{i}^{\beta}\right) \backslash \bigcup_{\xi \in \check{F}_{i}^{\beta}} h(\xi)=h\left(\dot{x}_{i}^{\beta}\right) \backslash \bigcup_{\xi \in \dot{F}_{i}^{\beta}} h(\xi),
$$

contradizendo a hipótese.

\subsection{Conseqüências topológicas e geométricas}

Primeiramente, vejamos algumas outras propriedades topológicas de $K_{2}$ :

Teorema 4.4. Em $V[G], K_{2}$ é um espaço compacto e disperso, hereditariamente separável de altura $\omega_{2}$.

Demonstração. Já vimos que $K_{2}$ é compacto pela própria definição e $K_{2}$ é disperso pelo Teorema 2.10. Segue do Teorema 4.3 que $K_{2}$ é hereditariamente separável e, portanto, resta verificar que $h t\left(K_{2}\right)=\omega_{2}$. 
Primeiramente, observe que para todo $\alpha<h t\left(K_{2}\right)$, como $K_{2}$ é hereditariamente separável, $K_{2}^{(\alpha)}$ é separável. Além disso, pela própria definição $(1.23), K_{2}^{(\alpha)} \backslash K_{2}^{(\alpha+1)}$ é o conjunto dos pontos isolados de $K_{2}^{(\alpha)}$ e, como $K_{2}^{(\alpha)}$ é separável, temos que $\left|K_{2}^{(\alpha)} \backslash K_{2}^{(\alpha+1)}\right| \leq$ $\aleph_{0}$.

Daí, para qualquer $\alpha<\omega_{2}$,

$$
\left|K_{2} \backslash K_{2}^{(\alpha)}\right|=\left|\bigcup_{\beta<\alpha} K_{2}^{(\beta)} \backslash K_{2}^{(\beta+1)}\right| \leq \aleph_{1}
$$

e, portanto, $\left|K_{2}^{(\alpha)}\right|=\aleph_{2}$. Logo, $h t\left(K_{2}\right) \geq \omega_{2}$.

Por outro lado, para todo $\alpha \in L_{2}=K_{2} \backslash\{*\}$, temos que $\min K_{2}^{(\alpha)}$ é um ponto isolado de $K_{2}^{(\alpha)}$ e, portanto, $\min K_{2}^{(\alpha)} \notin K_{2}^{(\alpha+1)}$. Por indução, segue que $\min K_{2}^{(\alpha+1)} \geq \alpha+1$. Logo,

$$
\bigcap_{\alpha<\omega_{2}} K_{2}^{(\alpha)}=\{*\}
$$

e então, $h t\left(K_{2}\right)=\omega_{2}$, concluindo a demonstração.

Lembramos mais uma vez que Just [35] mostrou que no modelo de Cohen não existem espaços compactos, dispersos, de largura enumerável e altura $\omega_{2}$. Por outro lado, Baumgartner e Shelah [5] mostraram a consistência da existência de um espaço com estas propriedades. $K_{2}$ é um espaço com propriedades mais fortes que aquelas do espaço de [5] e que, portanto, não existe no modelo de Cohen.

Segue do resultado acima o seguinte:

Corolário 4.5. Em $V[G], K_{2}$ é um espaço compacto tal que $h d\left(K_{2}\right)=\aleph_{0}$ e $h L\left(K_{2}\right)=\aleph_{2}$. Portanto, $h L\left(K_{2}\right) \not \leq h d\left(K_{2}\right)^{+}$.

Demonstração. Já vimos que $h d\left(K_{2}\right)=\aleph_{0}$ e sabemos que $h L\left(K_{2}\right) \leq\left|K_{2}\right|=\aleph_{2}$.

Por outro lado, pelo teorema anterior temos que a família

$$
\mathscr{C}=\left\{K_{2} \backslash K_{2}^{(\alpha)}: \alpha<\omega_{2}\right\}
$$

é um recobrimento aberto de $K_{2} \backslash\{*\}$ e observe que se $X \subseteq \omega_{2}$ é tal que $|X|<\aleph_{2}$, então 
existe $\gamma \in \omega_{2}$ tal que $X<\gamma$. Daí,

$$
\bigcup\left\{K_{2} \backslash K_{2}^{(\alpha)}: \alpha \in X\right\} \subseteq K_{2} \backslash K_{2}^{(\gamma)}
$$

e, como vimos na demonstração do teorema anterior,

$$
\left|K_{2} \backslash K_{2}^{(\gamma)}\right| \leq \aleph_{1}
$$

Ou seja, $\mathscr{C}$ é um recobrimento aberto de $K_{2} \backslash\{*\}$ sem subrecobrimento de cardinalidade menor ou igual a $\aleph_{1}$. Portanto, $h L\left(K_{2}\right)=\aleph_{2}$.

Lembramos que Shapirovskiı̆ [62] mostrou em ZFC que, para todo espaço compacto $K$, $h d(K) \leq h L(K)^{+}($Teorema 1.15).

Passemos, então, às propriedades do espaço de Banach $C\left(K_{2}\right)$. Assim como no capítulo anterior, podemos novamente concluir o seguinte:

Teorema 4.6. Em $V[G], C\left(K_{2}\right)$ é um espaço de densidade $\aleph_{2}$ que não admite renormação Fréchet-diferenciável e não possui nenhum sistema biortogonal não-enumerável.

Demonstração. O fato que $C\left(K_{2}\right)$ tem densidade $\aleph_{2}$ segue do Teorema 2.11, assim como o fato que $K_{2}$ tem peso $\aleph_{2}$.

Pelo Teorema 4.3, temos que $h d\left(K_{2}^{n}\right)=\aleph_{0}$ para todo $n \in \mathbb{N}$. Portanto, pelo Corolário 1.45, temos que $C\left(K_{2}\right)$ não admite uma renormação Fréchet-diferenciável e não possui nenhum sistema biortogonal não-enumerável.

Assim, $C\left(K_{2}\right)$ é o primeiro exemplo consistente de um espaço de Banach $C(K)$ de densidade estritamente maior que $\aleph_{1}$ sem nenhum sistema biortogonal não-enumerável. Lembramos que, pelo Teorema 1.46 de Todorcevic, todo espaço de Banach da forma $C(K)$ de densidade estritamente maior que $\aleph_{1}$ possui um sistema semi-biortogonal não-enumerável. Nosso exemplo mostra que não podemos generalizar esse resultado em ZFC, substituindo a existência de um sistema semi-biortogonal não-enumerável pela existência de um sistema biortogonal não-enumerável. 


\section{Apêndice A}

\section{Pântanos e a propriedade $\Delta$}

Nosso objetivo aqui é apresentar a demonstração de que é consistente com ZFC que existe uma função $f:\left[\omega_{2}\right]^{2} \rightarrow\left[\omega_{2}\right]^{\leq \aleph_{0}}$ tal que para todo $\xi<\eta<\omega_{2}, f(\{\xi, \eta\}) \subseteq \xi$ e se $A$ é uma família não-enumerável de subconjuntos finitos de $\omega_{2}$ que forma um $\Delta$-sistema, então existem $a, b \in A$ e uma bijeção $e: a \rightarrow b$ que preserva ordem constante em $a \cap b$ e tal que para todo $\xi \in a, \xi \leq e(\xi)$ e para todo $\zeta \in a \cap b$, todo $\xi \in a \backslash b$ e todo $\eta \in b \backslash a$ :

(i) se $\zeta<\xi$, então $f(\{\zeta, \eta\}) \subseteq f(\{\xi, \eta\})$;

(ii) se $\zeta<\eta$, então $f(\{\zeta, \xi\}) \subseteq f(\{\xi, \eta\})$;

(iii) $a \cap \xi \cap \eta \subseteq f(\{\xi, \eta\})$.

Lembramos que no Capítulo 4 , o espaço $L_{2}$ é definido num modelo obtido por forcing e que assumimos a existência dessa função no modelo inicial (veja a Definição 4.1). Assim, nosso objetivo aqui é apresentar uma demonstração do Teorema A.22, um resultado não publicado de Koszmider.

Para isso, vamos introduzir, na Seção A.1, a noção de $(\kappa, 1)$-pântanos simplificados $((\kappa, 1)$-simplified morasses $)$ e provar algumas propriedades básicas desses objetos. Na seção A.2, apresentamos a noção de forcing $\mathcal{F}$-próprio, onde $\mathcal{F} \subseteq\left[\omega_{2}\right]^{\leq \aleph_{0}}$ e mostramos que se $\mathcal{F}$ é um conjunto estacionário, então todo forcing $\mathcal{F}$-próprio preserva $\omega_{1}$. Finalmente, na Seção A.3, definimos um forcing $\mathbb{P}$ e, utilizando os resultados das seções anteriores, mostramos 
que $\mathbb{P}$ força a existência da função $f$ com as propriedades que queremos.

A noção de $(\kappa, 1)$-pântanos simplificados foi introduzida por Velleman [66] e [67].

\section{A.1 Pântanos simplificados}

Notação A.1. Dada uma familia de conjuntos $\mathcal{F}$ e um conjunto $X$, denotamos por $\mathcal{F} \mid X=$ $\{Y \in \mathcal{F}: Y \subsetneq X\}$.

Definição A.2 (Velleman [66], [67]). Seja $\kappa$ um cardinal regular. Um $(\kappa, 1)$-pântano simplificado é uma família $\mathcal{F} \subseteq\left[\kappa^{+}\right]^{<\kappa}$ que satisfaz as seguintes condições:

1. $\mathcal{F}$ é bem-fundada com relação à inclusão;

2. para todo $X \in \mathcal{F}$, temos que $|\mathcal{F}| X \mid<\kappa$;

3. para quaisquer $X, Y \in \mathcal{F}$, se $\operatorname{rank}(X)=\operatorname{rank}(Y)=\alpha$, então $X$ e $Y$ têm o mesmo tipo de ordem (i.e., ambos são ordem-isomorfos a um ordinal $\theta_{\alpha}$ ) e se $f_{X Y}$ é a única bijeção de $X$ sobre $Y$ que preserva ordem, então $\mathcal{F} \mid Y=\left\{f_{X Y}[Z]: Z \in \mathcal{F} \mid X\right\}$;

4. $\mathcal{F}$ é um conjunto dirigido com relação à inclusão, ou seja, para quaisquer $X, Y \in \mathcal{F}$, existe $Z \in \mathcal{F}$ tal que $X, Y \subseteq Z$;

5. $\mathcal{F}$ é localmente quase dirigido, ou seja, para todo $X \in \mathcal{F}$,

a) ou $\mathcal{F} \mid X$ é dirigido;

b) ou existem $X_{1}, X_{2} \in \mathcal{F}$ tais que $\operatorname{rank}\left(X_{1}\right)=\operatorname{rank}\left(X_{2}\right)$ e $X=X_{1} * X_{2}$, i.e., $X=$ $X_{1} \cup X_{2}, X_{1} \cap X_{2}<X_{1} \backslash X_{2}<X_{2} \backslash X_{1}$ e $\mathcal{F}|X=\mathcal{F}| X_{1} \cup \mathcal{F} \mid X_{2} \cup\left\{X_{1}, X_{2}\right\} ;$

6. $\bigcup \mathcal{F}=\kappa^{+}$.

Lema A.3 (Velleman [66], [67]). Seja $\mathcal{F}$ um $(\kappa, 1)$-pântano simplificado. Sejam $X, Y \in \mathcal{F}$ tais que $\operatorname{rank}(X)=\operatorname{rank}(Y)$ e $\alpha \in X \cap Y$. Então $X \cap \alpha=Y \cap \alpha$.

Demonstração. Seja $\mathcal{F}$ um $(\kappa, 1)$-pântano simplificado. Pela condição 4 da Definição A.2, $\mathcal{F}$ é dirigido e, portanto, vamos fazer a demonstração por indução no $\operatorname{rank}(Z)$, onde $X, Y \subseteq$ 
$Z \in \mathcal{F}$. Observe que $\mathcal{F}$ satisfaz a condição 5 da Definição A.2 e consideremos os seguintes casos:

Caso 1. $\mathcal{F} \mid Z$ é dirigido.

Neste caso, existe $Z_{1} \in \mathcal{F} \mid Z$ tal que $X, Y \subseteq Z_{1} \subsetneq Z$. Daí, $\operatorname{rank}\left(Z_{1}\right)<\operatorname{rank}(Z)$ e, pela hipótese indutiva, concluímos o resultado.

Caso 2. Existem $Z_{1}, Z_{2} \in \mathcal{F}$ tal que $Z=Z_{1} * Z_{2} \operatorname{erank}\left(Z_{1}\right)=\operatorname{rank}\left(Z_{2}\right)$.

Aqui, se $X, Y \subseteq Z_{i}$ para $i=1$ ou $i=2$, então, pela hipótese indutiva, concluímos o resultado. Senão, como $Z=Z_{1} * Z_{2}$, segue que $X \subseteq Z_{1}$ e $Y \subseteq Z_{2}$. Logo, se $\alpha \in$ $X \cap Y$, então $\alpha \in Z_{1} \cap Z_{2}$ e daí, segue da condição 3 da Definição A.2 que $f_{Z_{1} Z_{2}}[X] \subseteq$ $Z_{2}$ e $\operatorname{rank}\left(f_{Z_{1} Z_{2}}[X]\right)=\operatorname{rank}(X)=\operatorname{rank}(Y)$. Como $f_{Z_{1} Z_{2}}$ preserva a ordem, segue que $f_{Z_{1} Z_{2}} \mid Z_{1} \cap Z_{2}=i d_{Z_{1} \cap Z_{2}}$. Logo, $\alpha \in f_{Z_{1} Z_{2}}[X]$ e, pela hipótese indutiva, temos que $f_{Z_{1} Z_{2}}[X] \cap$ $\alpha=Y \cap \alpha$ e $f_{Z_{1} Z_{2}}[X] \cap \alpha=X$, concluindo a demonstração.

Lema A.4. Seja $\mathcal{F}$ um $(\kappa, 1)$-pântano simplificado. Então temos:

a) para todo $X \in \mathcal{F}$ e todo $\alpha<h t(\mathcal{F})$, se $\operatorname{rank}(X)<\alpha$, então existe $Z \in \mathcal{F}$ tal que $\operatorname{rank}(Z)=\alpha$ e $X \subseteq Z$;

b) para todo $Y \in \mathcal{F}$, todo $X \in \mathcal{F} \mid Y$ e todo $\operatorname{rank}(X)<\alpha<\operatorname{rank}(Y)$, existe $Z \in \mathcal{F}$ tal que $\operatorname{rank}(Z)=\alpha$ e $X \subseteq Z \subseteq Y$.

Observe que segue de b) que $h t(\mathcal{F}) \leq \kappa$.

Demonstração. Primeiramente, sejam $X \in \mathcal{F}$ e $\alpha<h t(\mathcal{F})$. Fixe $Y^{\prime} \in \mathcal{F}$ tal que $\operatorname{rank}\left(Y^{\prime}\right) \geq$ $\alpha$ e pela condição 4 da Definição A.2, existe $Y \in \mathcal{F}$ tal que $X, Y^{\prime} \subseteq Y$ de rank minimal tal que $X \subseteq Y$.

Observe que $\operatorname{rank}(Y) \geq \alpha$. Mostremos que não podemos ter que $\operatorname{rank}(Y)>\alpha$, concluindo, portanto, a demonstração de a). Pela condição 5 da Definição A.2 para $Y$, temos dois casos:

Caso 1. $\mathcal{F} \mid Y$ é dirigido. 
Neste caso, existe $Z \in \mathcal{F} \mid Y$ tal que $\operatorname{rank}(Z)=\alpha$, já que $\operatorname{rank}(Y)>\alpha$. Seja $Z^{\prime} \in \mathcal{F} \mid Y$ tal que $X, Z \subseteq Z^{\prime}$ e observe que $\operatorname{rank}\left(Z^{\prime}\right) \geq \alpha$, contradizendo a minimalidade de $\operatorname{rank}(Y)$.

Caso 2. Existem $Y_{1}, Y_{2} \in \mathcal{F}$ tais que $Y=Y_{1} * Y_{2}$ e $\operatorname{rank}\left(Y_{1}\right)=\operatorname{rank}\left(Y_{2}\right)$.

Note que $\alpha<\operatorname{rank}(Y)=\operatorname{rank}\left(Y_{i}\right)+1$ e, portanto, $\operatorname{rank}\left(Y_{i}\right) \geq \alpha$. Daí, ou $X \in \mathcal{F} \mid Y_{1}$, ou $X \in \mathcal{F} \mid Y_{2}$ e temos, também, uma contradição com a minimalidade do $\operatorname{rank}(Y)$ e concluímos a demonstração de a).

Sejam agora $\alpha<h t(\mathcal{F}), X, Y \in \mathcal{F}$ e $X \subseteq Y$ tais que $\operatorname{rank}(X)<\alpha<\operatorname{rank}(Y)$. Por a), temos que existem $Z_{1}, Z_{2} \in \mathcal{F}$ tais que $X \subseteq Z_{1} \subseteq Z_{2}, \operatorname{rank}\left(Z_{1}\right)=\alpha$ e $\operatorname{rank}\left(Z_{2}\right)=\operatorname{rank}(Y)$. Considere $f_{Z_{2} Y}$ e temos que $f_{Z_{2} Y}[X] \subseteq f_{Z_{2} Y}\left[Z_{1}\right], f_{Z_{2} Y}\left[Z_{1}\right] \in \mathcal{F}$ e $\operatorname{rank}\left(f_{Z_{2} Y}\left(Z_{1}\right)\right)=\alpha$.

É suficiente mostrar que $f_{Z_{2} Y}(X)=X$, i.e., $f_{Z_{2} Y}(\alpha)=\alpha$ para todo $\alpha \in X$. Mas se $\alpha \in X$ e $\alpha \in Z_{2} \cap Y$, pelo Lema A.3, temos que $\operatorname{ordtp}\left(\alpha \cap Z_{2}\right)=\operatorname{ordtp}(\alpha \cap Y)$, já que $f_{Z_{2} Y}$ preserva ordem e, portanto, $f_{Z_{2} Y}(\alpha)=\alpha$.

Definição A.5. Seja $\mathcal{F}$ um $(\kappa, 1)$-pântano simplificado e seja $\alpha \in \kappa^{+}$. A seqüência $\left(\mathcal{F}_{\xi}(\alpha)\right)_{\xi<\kappa}$ é chamada de $\alpha$-seqüência (com respeito a $\mathcal{F}$ ) se para todo $\xi \in \kappa$ temos que existem $X_{\xi} \in \mathcal{F}$ tal que $\operatorname{rank}\left(X_{\xi}\right)=\xi$ e $\alpha \in X_{\xi}$ tal que $\mathcal{F}_{\xi}(\alpha)=X_{\xi} \cap \alpha$ ou $\mathcal{F}_{\xi}(\alpha)=\emptyset$.

Lema A.6. Seja $\mathcal{F}$ um $(\kappa, 1)$-pântano simplificado. Se $\alpha \in \kappa^{+}$, então a $\alpha$-seqüência é a única seqüência não-decrescente em $[\alpha]^{<\kappa}$ cuja união é igual a $\alpha$.

Demonstração. Sejam $\xi<\xi^{\prime}<\kappa$.

Se $\mathcal{F}_{\xi}(\alpha) \neq \emptyset$, então, pelo Lema A.4, existe $Z \in \mathcal{F}$ tal que $\operatorname{rank}(Z)=\xi^{\prime}$ e $\mathcal{F}_{\xi}(\alpha) \subseteq Z$. Segue do Lema A.3 que $Z \cap \alpha=\mathcal{F}_{\xi^{\prime}}(\alpha)$ e, assim, $\mathcal{F}_{\xi^{\prime}}(\alpha) \supseteq \mathcal{F}_{\xi}(\alpha)$.

Finalmente, as condições 4 e 6 da Definição A.2 garantem que $\bigcup_{\xi<\kappa} \mathcal{F}_{\xi}(\alpha)=\alpha$ e a unicidade segue do Lema A.3.

Definição A.7. $\mathcal{F}$ é um conjunto estacionário codificador se é um subconjunto estacionário de $\left[\omega_{2}\right]^{\leq \aleph_{0}}$ e existe uma função injetora $c: \mathcal{F} \rightarrow \omega_{2}$ tal que para quaisquer $X, Y \in \mathcal{F}$, se $X \subsetneq Y$, então $c(X) \in Y$.

Lema A.8 (folclore). Suponha que $\mathcal{F} \subseteq\left[\omega_{2}\right]^{\leq \aleph_{0}}$ é um conjunto estacionário codificador e $\mathcal{F} \in M \prec H\left(\omega_{3}\right),|M|=\aleph_{0}, M \cap \omega_{2} \in \mathcal{F}$. Se $X \in \mathcal{F}$ e $X \subsetneq M$, então $X \in M$. 
Demonstração. Suponha que $X \in \mathcal{F}$ e $X \subsetneq M$. Como $\mathcal{F} \in M \prec H\left(\omega_{3}\right)$, temos que, em $M$, $\mathcal{F}$ é um conjunto estacionário codificador, de forma que existe $c: \mathcal{F} \rightarrow \omega_{2}$ que testemunha esse fato em $M$. Em particular, $\alpha=c(X) \in M \cap \omega_{2}$ e então, em $M, c^{-1}(\alpha)$ é um subconjunto enumerável de $\omega_{2}$. Daí, $\left.X \in M \cap\left[\omega_{2}\right]\right]^{\leq \aleph_{0}}$, como queríamos.

Lema A.9. Suponha que $\mathcal{F} \in M \prec H\left(\omega_{3}\right),|M|=\aleph_{0}, X=M \cap \omega_{2} \in \mathcal{F}$. Então $\operatorname{rank}(X)=$ $M \cap \omega_{1}$.

Demonstração. Seja $\delta=\operatorname{rank}(X)$.

Se $\delta \in M$, então temos, em $M, Y \in \mathcal{F}$ tal que $\operatorname{rank}(Y)=\delta$. Pela condição 3 da Definição A.2, temos que existe um isomorfismo entre $\mathcal{F} \mid Y$ e $\mathcal{F} \mid X$, contradizendo a condição 1 da Definição A.2.

Mas observe que $M$ contém todos os ordinais menores que $\delta$ e, portanto, todos os elementos de $\mathcal{F}$ de rank menor que $\delta$. Logo, todos os elementos de $\mathcal{F}$ de rank menor que $\delta$ estão incluídos em $M \cap \omega_{2}=X$, de forma que $\operatorname{rank}(X) \geq \delta$.

Lema A.10. Suponha que $\mathcal{F} \subseteq\left[\omega_{2}\right]^{\leq \aleph_{0}}$ é um conjunto estacionário codificador e $\mathcal{F} \in M \prec$ $H\left(\omega_{3}\right),|M|=\aleph_{0}, M \cap \omega_{2}=X_{0} \in \mathcal{F}$. Seja $Y \in \mathcal{F}$ tal que $\operatorname{rank}(Y)<M \cap \omega_{1}=\delta$. Então existe $Z(Y) \in M$ tal que:

1) $Y \cap X_{0} \subseteq Z(Y)$;

2) $\operatorname{rank}(Z(Y))=\operatorname{rank}(Y)$.

Demonstração. Pelo Lema A.4, seja $Y^{\prime} \in \mathcal{F}$ tal que $Y^{\prime} \supseteq Y$ e $\operatorname{rank}\left(Y^{\prime}\right)=\operatorname{rank}\left(X_{0}\right)=\delta$. Daí, pela condição 3 da Definição A.2, $\mathcal{F} \mid Y^{\prime}$ é isomorfo a $\mathcal{F} \mid X_{0}$ de forma que existe uma cópia $Z(Y)=f_{Y^{\prime} X_{0}}[Y]$ de $Y$ abaixo de $X_{0}$. Note que $Y \cap X_{0} \subseteq Y^{\prime} \cap X_{0}$ e $f_{Y^{\prime} X_{0}}$ é constante em $Y^{\prime} \cap X_{0}$. Daí, $Y \cap X_{0}=Y \cap M \subseteq Z(Y)$. Agora, pelo Lema A.8, segue de $Z(Y) \in \mathcal{F} \mid X_{0}$ que $Z(Y) \in M$.

\section{A.2 Forcings $\mathcal{F}$-próprios}

Definição A.11. Seja $\mathcal{F} \subseteq\left[\omega_{2}\right]^{\leq \aleph_{0}}$. Dizemos que um forcing $\mathbb{P}$ é $\mathcal{F}$-próprio se existem $\theta>\left(2^{|\mathbb{P}|}\right)^{+}$e um conjunto fechado e ilimitado $\mathcal{C} \subseteq[H(\theta)] \leq \aleph_{0}$ tais que se $p \in M \in \mathcal{C}$ e 
$M \cap \omega_{2} \in \mathcal{F}$, então existe $p_{0} \leq p(\mathbb{P}, M)$-genérico, i.e., $D \cap M$ é pré-denso abaixo de $p_{0}$ para todo $D \in M$ denso em $\mathbb{P}$.

Lema A.12. Se $\mathcal{F} \subseteq\left[\omega_{2}\right]^{\leq \aleph_{0}}$ é um conjunto estacionário e $\mathbb{P}$ é um forcing $\mathcal{F}$-próprio, então $\mathbb{P}$ preserva $\omega_{1}$.

Demonstração. A demonstração é uma versão da demonstração de Shelah para a preservação de $\omega_{1}$ por forcings próprios (veja [4] ou [63]).

Definição A.13. Seja $\mathbb{P}$ um forcing e $q \in \mathbb{P}$. Suponha que $M \prec H(\theta)$ e $\mathbb{P}, \pi_{1}, \ldots, \pi_{k} \in M$. Dizemos que uma fórmula $\phi\left(x_{0}, x_{1}, \ldots, x_{k}\right)$ bem-reflete $q$ em $\left(M ; \pi_{1}, \ldots, \pi_{k}\right)$ se as seguintes condições são satisfeitas:

i) $H(\theta)$ satisfaz $\phi\left(q, \pi_{1}, \ldots, \pi_{k}\right)$;

ii) se $s \in M$ é tal que $M$ satisfaz $\phi\left(s, \pi_{1}, \ldots, \pi_{k}\right)$, então q e s são compatíveis.

Definição A.14. Sejam $\mathcal{F} \subseteq\left[\omega_{2}\right]^{\leq \aleph_{0}}$ e $\mathbb{P}$ um forcing. Dizemos que $\mathbb{P}$ é simplesmente $\mathcal{F}$ próprio se existe $\theta$ tal que se $p \in \mathbb{P}$ e $M \prec H(\theta)$ são tais que $p, \mathbb{P}, \mathcal{F} \in M$ e $M \cap \lambda \in \mathcal{F}$, então existe $p_{0} \leq p$ tal que para todo $q \geq p_{0}$, existem $\pi_{1}, \ldots, \pi_{k} \in M$ e uma fórmula $\phi\left(x_{0}, x_{1}, \ldots, x_{k}\right)$ que bem-reflete q em $\left(M, \pi_{1}, \ldots, \pi_{k}\right)$.

Lema A.15. Se $\mathbb{P}$ é simplesmente $\mathcal{F}$-próprio, então $\mathbb{P}$ é $\mathcal{F}$-próprio.

Demonstração. Seja $\theta$ tal que se $p \in \mathbb{P}$ e $M \prec H(\theta)$ são tais que $p, \mathbb{P}, \mathcal{F} \in M$ e $M \cap \lambda \in \mathcal{F}$, então existe $p_{0} \leq p$ tal que para todo $q \geq p_{0}$, existem $\pi_{1}, \ldots, \pi_{k} \in M$ e uma fórmula $\phi\left(x_{0}, x_{1}, \ldots, x_{k}\right)$ que bem-reflete $q$ em $\left(M, \pi_{1}, \ldots, \pi_{k}\right)$.

Vejamos que $p_{0}$ é $(\mathbb{P}, M)$-genérico. Seja $D \in M$ um subconjunto denso de $\mathbb{P}$ e mostremos que $D \cap M$ é pré-denso abaixo de $p_{0}$. Seja $q \leq p_{0}$. Podemos assumir, sem perda de generalidade, que $q \in D$. Sejam $\pi_{1}, \ldots, \pi_{k} \in M$ e $\phi\left(x_{0}, x_{1}, \ldots, x_{k}\right)$ tais que $\phi\left(x_{0}, x_{1}, \ldots, x_{k}\right)$ bem-reflete $q$ em $\left(M, \pi_{1}, \ldots, \pi_{k}\right)$. Pela condição i) da Definição A.13, temos que $H(\theta)$ satisfaz $\phi\left(q, \pi_{1}, \ldots \pi_{k}\right)$. Pela elementaridade de $M$, temos que existe $s \in M \cap \mathbb{P}$ tal que $M$ satisfaz $\phi\left(s, \pi_{1}, \ldots \pi_{k}\right)$ e $s \in D$. Segue da Definição A.14 que $s$ e $q$ são compatíveis, de forma que $D \cap M$ contém uma condição compatível com $q$, mostrando, assim, que $D \cap M$ é pré-denso abaixo de $q$ e concluindo a demonstração. 


\section{A.3 Construindo a função $f$}

Nesta seção, fixemos um $\left(\omega_{1}, 1\right)$-pântano simplificado $\mathcal{F}$ que forma um subconjunto estacionário codificador de $\left[\omega_{2}\right]^{\leq \aleph_{0}}$. Vejamos a definição do forcing $\mathbb{P}$ que adiciona a função $f:\left[\omega_{2}\right]^{2} \rightarrow\left[\omega_{2}\right]^{\leq \aleph_{0}}$ que precisamos:

Definição A.16. Seja $\mathbb{P}$ o forcing formado pelas condições $p=\left(a_{p}, f_{p}, A_{p}\right)$, onde:

a) $a_{p} \in\left[\omega_{2}\right]^{<\aleph_{0}}$;

b) $f_{p}:\left[a_{p}\right]^{2} \rightarrow\left[\omega_{2}\right]^{<\omega}$;

c) $A_{p} \in[\mathcal{F}]^{<\aleph_{0}}$;

d) para quaisquer $\xi, \eta \in a_{p}$, $\xi \neq \eta$, temos que $f_{p}(\{\xi, \eta\}) \subseteq \bigcap\left\{X: X \in A_{p}, \xi, \eta \in X\right\} \cap$ $\min \{\xi, \eta\}$

ordenado por $p \leq q$ se $a_{p} \supseteq a_{q}, f_{p} \supseteq f_{q}$ e $A_{p} \supseteq A_{q}$.

Lema A.17. Assumindo a hipótese do contínuo, $\mathbb{P}$ é simplesmente $\mathcal{F}$-próprio.

Demonstração. Seja $\theta=\omega_{3}$ e sejam $p \in \mathbb{P}$ e $M \prec H(\theta)$ como na Definição A.16. A existência de $M$ segue de nossas hipóteses sobre $\mathcal{F}$.

Seja $X_{0}=M \cap \omega_{2}$ e defina $p_{0}=\left(a_{p}, f_{p}, A_{p} \cup\left\{X_{0}\right\}\right)$. Observe que $p_{0} \in \mathbb{P}$.

Agora, dado $q \leq p_{0}$, precisamos achar $\pi_{1}, \ldots, \pi_{k} \in M$ e uma fórmula $\phi\left(x_{0}, x_{1}, \ldots, x_{k}\right)$ que bem-reflete $q$ em $\left(M, \pi_{1}, \ldots, \pi_{k}\right)$

Defina $q \mid M=\left(a_{q} \cap M, f_{q} \mid M, A_{q} \cap M\right)$ e $\delta=M \cap \omega_{1}$. Segue do Lema A.9 que $\delta=$ $\operatorname{rank}(M)$. Além disso, como $\mathcal{F}$ é um conjunto estacionário codificador, segue do Lema A.8 que $A_{q} \cap M=A_{q \mid M}=\left\{X \in A_{q}: X \in M, X \subsetneq X_{0}\right\}$. O fato que [M] $\leq^{\leq \aleph_{0}} \subseteq M$ implica que $a_{q \mid M}, A_{q \mid M} \in M$. Mais ainda, como $q \in \mathbb{P}$ e $X_{0}=M \cap \omega_{2}$, segue da condição d) da Definição A.16 que $f_{q \mid M} \in M$, ou seja, temos que $q \mid M \in M \cap \mathbb{P}$.

É claro que $q \mid M \leq p$.

Segue do Lema A.10 e do fato que $[M]^{\leq \aleph_{0}} \subseteq M$ que existe uma família $\mathcal{Z} \in M, \mathcal{Z} \subseteq \mathcal{F}$, tal que $\bigcup\left\{X \cap M: \operatorname{rank}(X)<\delta, X \in A_{q}\right\} \subseteq \bigcup \mathcal{Z}$. Como $\mathcal{F}$ é cofinal (estacionário) em 
$\left[\omega_{2}\right] \leq \aleph_{0}$ e pela elementaridade de $M$, existe $Z \in \mathcal{F} \cap M$ tal que $\bigcup\{X \cap M: \operatorname{rank}(X)<$ $\left.\delta, X \in A_{q}\right\} \subseteq Z$.

Seja $\phi\left(x_{0}, x_{1}, x_{2}, x_{3}, x_{4}\right)$ a fórmula que diz que $x_{0}$ é uma condição da ordem parcial $x_{4}$ que estende a condição $x_{3}$ e tal que a diferença entre a primeira coordenada de $x_{0}$ e $x_{2}$ é disjunta de $x_{1}$.

Afirmação. $\phi\left(x_{0}, x_{1}, x_{2}, x_{3}, x_{4}\right)$ é uma fórmula que bem-reflete $q$ em $\left(M, Z, a_{q \mid M}, q \mid M, \mathbb{P}\right)$. Além disso, se $M$ satisfaz $\phi\left(s, Z, a_{q \mid M}, q \mid M, \mathbb{P}\right), r \leq q$, s e $h:\left(a_{s} \backslash a_{q \mid M}\right) \otimes\left(a_{q} \backslash a_{q \mid M}\right) \rightarrow$ $\left[\omega_{2}\right]^{<\aleph_{0}}$ é uma função que satisfaz

$$
h(\{\xi, \eta\}) \subseteq \min (\{\xi, \eta\}) \cap \bigcap\left\{X \in A_{q}: \xi, \eta \in X, \xi \neq \eta, \operatorname{rank}(X) \geq \delta\right\}
$$

(convencionando que a intersecção da família vazia é $\omega_{2}$ ), podemos supor, sem perda de generalidade, que $f_{r} \mid\left(a_{s} \backslash a_{q \mid M}\right) \otimes\left(a_{q} \backslash a_{q \mid M}\right)=h$.

Demonstração da Afirmação. É claro que $H(\theta)$ satisfaz $\phi\left(q, Z, a_{q \mid M}, q \mid M, \mathbb{P}\right)$. Pela elementaridade de $M$, existe $s \in M$ tal que $\phi\left(s, Z, a_{q \mid M}, q \mid M, \mathbb{P}\right)$, ou seja, $s$ é uma condição de $\mathbb{P}$ que estende $q \mid M$ e $a_{s} \backslash a_{q \mid M} \cap Z=\emptyset$. Seja $h:\left(a_{s} \backslash a_{q \mid M}\right) \otimes\left(a_{q} \backslash a_{q \mid M}\right) \rightarrow\left[\omega_{2}\right]^{<\aleph_{0}}$ satisfazendo

$$
h(\{\xi, \eta\}) \subseteq \min (\{\xi, \eta\}) \cap \bigcap\left\{X \in A_{q}: \xi, \eta \in X, \xi \neq \eta, \operatorname{rank}(X) \geq \delta\right\}
$$

Defina $r=\left(a_{r}, f_{r}, A_{r}\right)$ da seguinte maneira: $a_{r}=a_{s} \cup a_{q}, f_{r}=f_{s} \cup f_{q} \cup h$ e $A_{r}=A_{s} \cup A_{q}$. É fácil ver que $r$ satisfaz as condições a), b) e c) da Definição A.16. Vejamos que $r$ satisfaz a condição d) da Definição A.16: $\operatorname{sejam~} \xi, \eta \in a_{r}, \xi \neq \eta$, e $X \in A_{r}$.

Caso 1. $\xi, \eta \in a_{s}$ e $X \in A_{s}$.

Este caso é trivial, já que $s \in \mathbb{P}$.

Caso 2. $\xi, \eta \in a_{q}$ e $X \in A_{q}$. 
Este caso é trivial, já que $q \in \mathbb{P}$.

Caso 3. $\xi, \eta \in a_{s}$ e $X \in A_{q}$.

Como $M$ satisfaz $\phi\left(s, Z, a_{q \mid M}, q \mid M, \mathbb{P}\right)$, temos duas possibilidades: ou $\operatorname{rank}(X) \geq \delta$ ou $\operatorname{rank}(X)<\delta$.

Se $\operatorname{rank}(X) \geq \delta=M \cap \omega_{1}=\operatorname{rank}(M)$, temos que $f_{r}(\{\xi, \eta\})=f_{s}(\{\xi, \eta\}) \subseteq M \cap$ $\min \{\xi, \eta\} \subseteq X$ pelo Lema A.6. Daí, como $s \in M$, segue que $M \cap \omega_{2} \in \mathcal{F}$ e temos que $r$ satisfaz a condição d) da Definição A.16.

Se, por outro lado, $\operatorname{rank}(X)<\delta$, pela definição de $\phi$ e $Z$ temos que $\xi, \eta \in a_{s} \cap a_{q}$ e segue do Caso 2 que $r$ satisfaz a condição d) da Definição A.16.

Caso 4. $\xi, \eta \in a_{q}$ e $X \in A_{s}$.

Aqui, temos que $\xi, \eta \in M$, ou seja, $\xi, \eta \in a_{s} \cap a_{q}$ e segue do Caso 1 que $r$ satisfaz a condição d) da Definição A.16.

Caso 5. $\xi \in a_{s} \backslash a_{q}$ e $\eta \in a_{q} \backslash a_{s}$.

Neste caso, temos que $X \in A_{q}$ e, pela definição de $\phi$ e $Z$, temos que $\operatorname{rank}(X) \geq \delta$. Mas $f_{r}(\{\xi, \eta\})=h(\{\xi, \eta\})$ e segue que $r$ satisfaz a condição d) da Definição A.16.

Concluímos assim a demonstração da afirmação e do lema.

Definição A.18. Dado $p \in P$, o suporte de $p$, supp $(p)$, é o conjunto $a_{p} \cup \bigcup A_{p}$.

Definição A.19. Dizemos que duas condições $p, q \in \mathbb{P}$ são isomorfas (via $\pi: \operatorname{supp}(p) \rightarrow$ $\operatorname{supp}(q))$ se $\pi: \operatorname{supp}(p) \rightarrow \operatorname{supp}(q)$ é uma bijeção que preserva ordem, constante em $\operatorname{supp}(p) \cap \operatorname{supp}(q)$ e tal que $\pi\left[a_{p}\right]=a_{q},\left\{\pi[X]: X \in A_{p}\right\}=A_{q}$ e para todo $\alpha, \beta \in a_{p}$, $f_{q}(\pi(\alpha), \pi(\beta))=f_{p}(\alpha, \beta)$.

Lema A.20. Sejam $p, q \in \mathbb{P}$ duas condições isomorfas via $\pi: \sup p(p) \rightarrow \operatorname{supp}(q)$. Seja $\Delta=\operatorname{supp}(p) \cap \operatorname{supp}(q)$ e seja $r \leq p$ tal que supp $(r) \cap \operatorname{supp}(q)=\Delta$. Se $h:\left(a_{r} \backslash \Delta\right) \otimes\left(a_{q} \backslash \Delta\right) \rightarrow$ $\left[\omega_{2}\right]^{<\aleph_{0}}$ é uma função tal que, para todo $\xi \in a_{r} \backslash \Delta$ e todo $\eta \in a_{q} \backslash \Delta, h(\{x i, \eta\}) \subseteq \min \{\xi, \eta\}$, existe $s \leq r, q$ tal que $a_{s}=a_{r} \cup a_{q}, A_{s}=A_{r} \cup A_{q}$ e $f_{s}=f_{r} \cup f_{q} \cup h$.

Demonstração. Precisamos verificar apenas que $s \in \mathbb{P}$. É claro que $s$ satisfaz as condições a), b) e c) da Definição A.16. Resta verificar que $s$ satisfaz a condição d) da Definição A.16. 
Sejam $\xi, \eta \in a_{s}, \xi \neq \eta$, e $X \in A_{s}$.

Caso 1. $\xi, \eta \in a_{r}$.

Se $X \in A_{r}$, segue diretamente do fato que $r \in \mathbb{P}$. Senão, $X \in A_{q}$ e $\xi, \eta \in X$. Daí, $\xi, \eta \in \Delta$ e, portanto, $\xi, \eta \in \pi^{-1}[X] \in A_{p} \subseteq A_{r}$ e como $r \in \mathbb{P}$, segue que a condição d) da Definição A.16 é satisfeita neste caso.

Caso 2. $\xi, \eta \in a_{q}$.

Se $X \in A_{q}$, segue diretamente do fato que $q \in \mathbb{P}$. Senão, $X \in A_{r}$ e $\xi, \eta \in X$. Daí, $\xi, \eta \in \Delta$ e, portanto, $\xi, \eta \in \pi^{-1}[X] \in A_{q}$ e como $q \in \mathbb{P}$, segue que a condição d) da Definição A.16 é satisfeita neste caso.

Caso 3. $\xi \in a_{r} \backslash a_{q}, \eta \in a_{q} \backslash a_{r}$.

Observe que não existe $X \in A_{s}$ tal que $\xi, \eta \in X$. Como, neste caso, $f_{s}(\{\xi, \eta\})=h(\{\xi, \eta\}) \subseteq$ $\min \{\xi, \eta\}$, segue que a condição d) da Definição A.16 também é satisfeita neste caso.

Lema A.21. Assumindo a hipótese do contínuo, o forcing $\mathbb{P}$ é $\omega_{2}$-c.c. e, portanto, $\mathbb{P}$ preserva cardinais.

Demonstração. Para cada $\alpha<\omega_{2}$, sejam $p_{\alpha}=\left(a_{\alpha}, f_{\alpha}, A_{\alpha}\right) \in \mathbb{P}$. Por um argumento usual de contagem, podemos garantir, usando a hipótese do contínuo, que existem $\alpha<\beta<\omega_{2}$ tais que $p_{\alpha}$ e $p_{\beta}$ são isomorfos. Daí, fixe $h:\left(a_{\alpha} \backslash a_{\beta}\right) \otimes\left(a_{\beta} \backslash a_{\alpha}\right) \rightarrow\left[\omega_{2}\right]^{<\aleph_{0}}$ arbitrária e defina $q=\left(a_{q}, f_{q}, A_{q}\right)$ por: $a_{q}=a_{\alpha} \cup a_{\beta}, f_{q}=f_{\alpha} \cup f_{\beta} \cup h$ e $A_{\alpha} \cup A_{\beta}$. Segue do lema anterior que $q \in \mathbb{P}$ e concluímos que $\mathbb{P}$ é $\omega_{2}$-c.c. e preserva, portanto, cardinais maiores ou iguais a $\aleph_{2}$.

Por outro lado, segue do Lema A.17, que $\mathbb{P}$ é simplesmente $\mathcal{F}$-próprio. Daí, pelo Lema A.15 que $\mathbb{P}$ é $\mathcal{F}$-próprio e, então, pelo Lema A.12, temos que $\mathbb{P}$ preserva $\aleph_{1}$. Portanto, temos que $\mathbb{P}$ preserva cardinais.

Teorema A.22. Em $V^{\mathbb{P}}$, existe uma função $f:\left[\omega_{2}\right]^{2} \rightarrow\left[\omega_{2}\right]^{\leq \aleph_{0}}$ tal que para todo $\xi<\eta<$ $\omega_{2}, f(\{\xi, \eta\}) \subseteq \xi$ e se $A$ é uma família não-enumerável de subconjuntos finitos de $\omega_{2}$ que forma um $\Delta$-sistema, então existem $a, b \in A$ e uma bijeção $e: a \rightarrow b$ que preserva ordem constante em $a \cap b$ e tal que para todo $\xi \in a, \xi \leq e(\xi)$ e para todo $\zeta \in a \cap b$, todo $\xi \in a \backslash b$ e 
todo $\eta \in b \backslash a$ :

(i) se $\zeta<\xi$, então $f(\{\zeta, \eta\}) \subseteq f(\{\xi, \eta\})$;

(ii) se $\zeta<\eta$, então $f(\{\zeta, \xi\}) \subseteq f(\{\xi, \eta\})$;

(iii) $a \cap \xi \cap \eta \subseteq f(\{\xi, \eta\})$.

Demonstração. Seja $G$ um filtro $\mathbb{P}$-genérico sobre $V$ e considere $f=\bigcup\left\{f_{p}: p \in G\right\}$. Seja $\dot{f}$ um nome para $f$.

Fixe uma família não-enumerável $\left\{\dot{a}_{\alpha}: \alpha<\omega_{1}\right\} \subseteq\left[\omega_{2}\right]^{n}$ de nomes para subconjuntos finitos de $\omega_{2}$ que formam um $\Delta$-sistema. Sem perda de generalidade, podemos assumir que existe $n \in \mathbb{N}$ e para todo $\alpha<\omega_{1}$ existem $\dot{a}_{1}^{\alpha}, \ldots, \dot{a}_{n}^{\alpha}$ nomes para elementos de $\omega_{2}$ tais que $\dot{a}_{\alpha}=\left\{\dot{a}_{1}^{\alpha}, \ldots, \dot{a}_{n}^{\alpha}\right\}$.

Seja $p \in P$. Considere $M \prec H\left(\omega_{3}\right)$ tal que $M \cap \omega_{2}=X_{0} \in \mathcal{F}, p, \mathbb{P}, \mathcal{F} \in M$ e $\left\{\dot{a}_{\alpha}: \alpha<\omega_{1}\right\} \in M$. Mostremos que existem $\alpha<\beta<\omega_{1}$ e $r \leq p$ tais que $r$ força que as condições i), ii) e iii) do enunciado são satisfeitas para $\dot{a}_{\alpha}$ e $\dot{a}_{\beta}$.

Primeiramente, seja $p_{0} \leq p$ dada por $a_{p}=a_{p_{0}}, f_{p}=f_{p_{0}}, A_{p}=A_{p_{0}} \cup\left\{X_{0}\right\}$.

Sejam $q \leq p_{0}$ e $\alpha \in \omega_{1}$ tais que existe $b$ tal que $b \backslash M \neq \emptyset, q$ força que $\dot{a}_{\alpha}=\check{b}$ e $b \subseteq a_{q}$. Podemos fazer isso já que $\left\{\dot{a}_{\alpha}: \alpha<\omega_{1}\right\}$ é uma seqüência de nomes para elementos de um $\Delta$-sistema não-enumerável de conjuntos e $|M|=\aleph_{0}$. Como na demonstração do Lema A.17, obtemos $s \geq q \mid M$ satisfazendo $\phi\left(s, Z, a_{q \mid M}, q \mid M, \mathbb{P}\right)$ da Afirmação do Lema A.17. Novamente, pela disjunção dos conjuntos e pela elementaridade de $M$, podemos obter $s$ e $\beta$ tais que $M$ satisfaz $\phi\left(s, Z, a_{q \mid M}, q \mid M, \mathbb{P}\right)$ e existe $a \in[M \backslash Z]^{<\aleph_{0}}$ tal que $q$ força que $\dot{a}_{\beta}=\check{a}$ e $a \subseteq a_{s}$.

Segue da Afirmação do Lema A.17 que podemos definir

$$
h(\{\xi, \eta\})=D \cap \min \{\xi, \eta\} \cap \bigcap\left\{X \in A_{q}: \xi, \eta \in X, \operatorname{rank}(X) \geq \delta\right\}
$$

para $\xi \in a \backslash b$ e $\xi \in b \backslash a$, onde

$$
D=(a \cup b) \cup \bigcup\left\{f_{r}(\{\zeta, \xi\}): \zeta \in a \cap b, \zeta<\eta\right\} \cup \bigcup\left\{f_{r}(\{\zeta, \eta\}): \zeta \in a \cap b, \zeta<\xi\right\},
$$


obtendo $r$ como no Lema A.17, $r \leq p$.

Resta verificar que as condições i), ii) e iii) do enunciado estão satisfeitas para $a, b$ e $f_{r}$ como acima. Isso é suficiente, já que $r$ força que $\dot{a}_{\alpha}=\check{b}, \dot{a}_{\beta}=\check{a}$ e $f_{r} \subseteq \dot{f}$. Sejam então $\xi \in a \backslash b$ e $\eta \in b \backslash a$ e note que segue do Lema A.6 que se $\xi, \eta \in X \in A_{q}$ e $\operatorname{rank}(X) \geq \delta$, então $X_{0} \cap \xi \subseteq X$.

Para verificar i), seja $\zeta \in a \cap b$ e suponha que $\zeta<\xi$. Observe que $\min \{\zeta, \eta\} \leq \min \{\xi, \eta\}$ e daí, pela definição de $f_{r}$, temos que $f_{r}(\{\xi, \eta\})=h(\{\xi, \eta\})$ e, portanto, $f_{r}(\{\zeta, \eta\}) \subseteq$ $D \cap \min \{\xi, \eta\}$. Seja agora $X \in A_{q}$ tal que $\xi, \eta \in X$ e $\operatorname{rank}(X) \geq \delta$ e mostremos que $f_{r}(\{\zeta, \eta\}) \subseteq X$. Como $\zeta \in a \cap \xi \subseteq M \cap \xi \subseteq X_{0} \cap \xi \subseteq X$ e como $r \leq q$ e $q \in \mathbb{P}$, segue que $f_{r}(\{\zeta, \eta\})=f_{q}(\{\zeta, \eta\}) \subseteq X$, como queríamos.

Para verificar ii), seja $\zeta \in a \cap b$ e suponha que $\zeta<\eta$. Observe que $\min \{\zeta, \xi\} \leq \min \{\xi, \eta\}$ e daí, pela definição de $f_{r}$, temos que $f_{r}(\{\xi, \eta\})=h(\{\xi, \eta\})$ e, portanto, $f_{r}(\{\zeta, \xi\}) \subseteq D \cap$ $\min \{\xi, \eta\}$. Seja agora $X \in A_{q}$ tal que $\xi, \eta \in X$ e $\operatorname{rank}(X) \geq \delta$ e mostremos que $f_{r}(\{\zeta, \xi\}) \subseteq$ $X$. Como $\zeta, \xi \in a \subseteq M \cap \omega_{2}=X_{0}$ e como $r \leq s$ e $s \in \mathbb{P}$, segue que $f_{r}(\{\zeta, \xi\})=f_{s}(\{\zeta, \xi\}) \subseteq$ $X_{0} \cap \min \{\zeta, \xi\} \subseteq X$, como queríamos.

Finalmente, observe que $a \cap \xi \cap \eta \subseteq D \cap \min \{\xi, \eta\}$ e como $a \cap \xi \subseteq M \cap \xi \subseteq X_{0} \cap \xi \subseteq X$, segue que $a \subseteq f_{r}(\{\xi, \eta\})$ e concluímos a demonstração de iii) e do teorema. 


\section{Apêndice B}

\section{Constructions génériques d'espaces d'Asplund C(K)}

Un espace de Banach $X$ est dit d'Asplund si toute fonction réelle continue et convexe, définie sur $X$, est Fréchet-différentiable en chaque point d'un sous-ensemble $G_{\delta}$ dense de $X$.

En 1975, Asplund [2] a montré que les espaces d'une certaine classe d'espaces de Banach qui inclut les espaces $c_{0}(\Gamma)$ (où $\Gamma$ est n'importe quel ensemble) et les espaces réflexifs ont cette propriété. Depuis, de nombreuses caractérisations des espaces d'Asplund sont apparues dans la littérature (voir, par exemple, [29], [42] et [46]) et ces espaces jouent, aujourd'hui, un rôle important dans la théorie des espaces de Banach et, plus particulièrement, dans la théorie des renormages.

Pour tout espace de Banach séparable $X$, une conjonction de résultats dûs à plusieurs mathématiciens, y compris Asplund, Gregory, Kadec, Klee, Namioka, Phelps et Stegall, parmi d'autres, implique que $X$ admet un renormage Fréchet-différentiable si et seulement si $X$ admet une fonction bosse Fréchet-différentiable et si et seulement si $X$ est un espace d'Asplund.

Il est alors naturel de poser la question suivante: que se passe-t-il dans le cas non séparable? 
Pour n'importe quel espace de Banach il est facile de construire, à partir d'une norme équivalente Fréchet-différentiable, une fonction bosse Fréchet-différentiable, et on peut montrer que si $X$ admet une fonction bosse Fréchet-différentiable, alors $X$ est un espace d'Asplund. Il reste donc à vérifier si les implications réciproques sont vraies.

La classe des espaces de Banach $C(K)$ fournit des exemples intéressants d'espaces de Banach non séparables (voir, par exemple, [9], [24], [39], [40], [48] et [52]). Ici, la situation est semblable: plusieurs exemples intéressants d'espaces d'Asplund non séparables sont des espaces $C(K)$. Namioka et Phelps [46] ont prouvé que pour tout espace compact $K$ (ou localement compact $L$ ), nous avons que $C(K)$ (ou $C_{0}(L)$ ) est d'Asplund si et seulement si $K$ (ou $L$ ) est clairsemé, c'est-à-dire, si tout sous-ensemble de $K$ (ou de $L$ ) possède des points isolés.

Les exemples d'espaces compacts clairsemés les plus simples à définir sont: le compactifié d'Alexandroff d'un espace discret; et un ordinal successeur avec la topologie de l'ordre. Mazurkiewicz et Sierpiński [44] ont montré que tout espace compact, métrique et clairsemé est homéomorphe à un ordinal dénombrable avec la topologie de l'ordre.

Toutefois, nous sommes intéressés par les espaces d'Asplund non séparables et, donc, nous ne considérons que les espaces compacts et clairsemés non métrisables. Il existe dans la littérature de nombreuses constructions de tels espaces qui ne sont pas homéomorphes à des ordinaux et qui sont construits par de méthodes ensemblistes: ([49], [17], [56], [28]) et même de tels espaces qui sont compacts ou localement compacts ([3], [38], [36]). Le premier exemple d'un espace compact et clairsemé dont la hauteur est $\aleph_{2}$ et la largeur est dénombrable est celui de Baumgartner et Shelah [5], obtenu par forcing.

Rappelons-nous que nous sommes intéressés par les propriétés des espaces d'Asplund $C(K)$ non séparables et retournons aux questions que nous nous sommes posées: est-ce que tout espace d'Asplund possède une norme équivalente Fréchet-différentiable ou admet une fonction bosse Fréchet-différentiable, comme chez les espaces d'Asplund séparables?

Haydon [25] construit un arbre $T$ tel que $C_{0}(T)$ n'admet pas de renormage Gâteaux- 
différentiable. Les arbres sont des espaces localement compacts et clairsemés et, donc, nous avons que $C_{0}(T)$ est un espace d'Asplund; puisque la Fréchet-différentiabilité d'une fonction implique sa Gâteaux-différentiabilité, un tel espace est un exemple d'espace d'Asplund sans renormage Fréchet-différentiable.

Par contre, Haydon [26] prouve que pour tout arbre $T, C_{0}(T)$ admet une fonction bosse Fréchet-différentiable, de sorte que l'exemple construit dans son travail précédent, aussi bien que ceux associés à n'importe quel arbre, ne répond pas à la question suivante, toujours sans réponse: existe-t-il un espace d'Asplund qui n'admet pas de fonction bosse Fréchetdifférentiable?

Dans l'espoir de répondre négativement à cette question par l'exemple d'un espace $C(K)$, le résultat de Haydon nous oblige à chercher des exemples d'espaces compacts (ou localement compacts) et clairsemés non métrisables, qui ne sont pas des arbres.

L'espace $C(K)$, où $K$ est le compact de Kunen (dont la construction est reproduite dans [48]), est un des espaces de fonctions continues les plus importants du point de vue de la théorie des espaces de Banach : $K$ est un espace compact clairsemé, construit sous l'hypothèse du continu et dont toute puissance finie est héréditairement séparable. On peut alors prouver que l'espace d'Asplund $C(K)$ correspondant n'admet pas de renormage Fréchet-différentiable ni de système biorthogonal non dénombrable (voir [31] et [47]). Cependant, on ne sait pas si $C(K)$ admet un renormage Gâteaux-différentiable ni si $C(K)$ admet une fonction bosse Fréchet-différentiable. Les espaces que nous étudions dans ce travail possèdent des propriétés similaires.

Notre but est de présenter une méthode de construction par forcing d'espaces compacts et clairsemés non métrisables $K$ et d'analyser les propriétés topologiques et géométriques des espaces d'Asplund $C(K)$ correspondants. Cette méthode est fondée sur l'espace construit par Rabus [53] pour montrer la consistance de l'existence d'un espace initialement $\omega_{1}$ compact de tightness dénombrable et non compact $^{1}$, ce qui répond à une question de Dow et van Douwen. L'idée de cette construction vient de celle de Baumgartner et Shelah [5]. Dans [33], Juhasz et Soukup font un abordage alternatif de la construction de Rabus, par

\footnotetext{
${ }^{1}$ N.B. De fait, Rabus [53] a construit une algèbre de Boole superatomique, dont l'espace de Stone possède un point distingué, de sorte que, si nous retirons ce point-là, l'espace obtenu a les propriétés mentionnées.
} 
un langage topologique au lieu du langage des algèbres de Boole.

Le travail est composé de quatre chapitres suivis d'une annexe.

\section{Définitions et résultats préliminaires}

Dans le premier chapitre nous dressons simplement une liste des résultats déjà connus qui seront utilisés dans les chapitres suivants.

\section{Constructions d'espaces clairsemés génériques}

Dans le deuxième chapitre, nous présentons la méthode de construction d'espaces compacts clairsemés que nous avons déjà mentionnée: nous définissons un forcing qui introduit une topologie sur un ordinal non dénombrable $\theta \leq \omega_{2}$, qui dépend, comme dans [33], d'une fonction qui associe un sous-ensemble dénombrable de $\theta$ à chaque paire de $\theta$. Les propriétés de $f$ ont une influence sur les propriétés de la topologie introduite $\operatorname{sur} \theta$ :

Définition 1. Soient $\theta \leq \omega_{2}$ un ordinal non dénombrable et $f:[\theta]^{2} \rightarrow[\theta] \leq \aleph_{0}$ une fonction telle que pour tous $\xi, \eta \in \theta, \xi \neq \eta, f(\{\xi, \eta\}) \subseteq \min \{\xi, \eta\}$. Soit $\mathbb{P}_{f}$ le forcing des conditions $p=\left(D_{p}, h_{p}, i_{p}\right)$, ò̀:

1. $D_{p} \in[\theta]^{<\aleph_{0}}$;

2. $h_{p}: D_{p} \rightarrow \wp\left(D_{p}\right)$ et pour tout $\xi \in D_{p}$, on a que $\max h_{p}(\xi)=\xi$;

3. $i_{p}:\left[D_{p}\right]^{2} \rightarrow\left[D_{p}\right]^{<\aleph_{0}}$ et pour tous $\xi, \eta \in D_{p}, \xi<\eta$, on a que:

(a) si $\xi \in h_{p}(\eta)$, alors $h_{p}(\xi) \backslash h_{p}(\eta) \subseteq \bigcup_{\gamma \in i_{p}(\{\xi, \eta\})} h_{p}(\gamma)$,

(b) si $\xi \notin h_{p}(\eta)$, alors $h_{p}(\xi) \cap h_{p}(\eta) \subseteq \bigcup_{\gamma \in i_{p}(\{\xi, \eta\})} h_{p}(\gamma)$,

(c) et $i_{p}(\{\xi, \eta\}) \subseteq f(\{\xi, \eta\})$;

ordonné par $p \leq q$ si $D_{p} \supseteq D_{q}$ et pour tout $\xi \in D_{q}, h_{p}(\xi) \cap D_{q}=h_{q}(\xi)$ et $\left.i_{p}\right|_{\left[D_{q}\right]^{2}}=i_{q}$. 
Ensuite nous montrons que ce forcing introduit une topologie sur $\theta$ de sorte que nous obtenons un espace compact clairsemé non métrisable: fixons $V$ un modèle et $G$ un filtre générique sur $\mathbb{P}_{f}$.

Définition 2. Pour chaque $\xi<\eta<\theta$, soit, dans $V[G]$,

$$
h(\xi)=\bigcup_{p \in G} h_{p}(\xi) \quad \text { et } \quad i(\{\xi, \eta\})=\bigcup_{p \in G} i_{p}(\{\xi, \eta\}) .
$$

Il est facile de voir que pour chaque $\xi<\theta, \xi=\max h(\xi)$ et que si pour n'importe quels $\xi<\eta<\theta$, on dénote

$$
h(\xi) * h(\eta)= \begin{cases}h(\xi) \backslash h(\eta) & \text { si } \xi \in h(\eta), \\ h(\xi) \cap h(\eta) & \text { si } \xi \notin h(\eta),\end{cases}
$$

alors $i(\{\xi, \eta\})$ est un sous-ensemble fini de $\xi$ tel que

$$
h(\xi) * h(\eta) \subseteq \bigcup_{\gamma \in i(\{\xi, \eta\})} h(\gamma)
$$

Dans $V[G]$, soit $L$ l'espace topologique générique $L=(\theta, \tau)$, où $\tau$ est la topologie sur $\theta$ qui possède l'ensemble

$$
\{h(\xi): \xi<\theta\} \cup\{L \backslash h(\xi): \xi<\theta\}
$$

comme sous-base. Nous appelons $h(\xi)$ voisinage générique de $\xi$.

Nous prouvons quelques propriétés du forcing et de l'espace $L$ qui seront utilisées dans les chapitres suivants.

Puis, nous prouvons deux lemmes combinatoires qui assurent l'existence d'amalgamations, c'est-à-dire, d'extensions communes à deux conditions spécifiques du forcing, sous quelques hypothèses concernant ces deux conditions: les Lemmes 2.13 et 2.14. Le Lemme 2.13 a des hypothèses assez fortes sur les conditions du forcing, mais qui assurent la possibilité de construire une amalgamation qui "copie une condition dans l'autre". Dans le Lemme 2.14, nous mixturons des idées des amalgamations introduites dans [33] et [53] et celles du Lemme 2.13. Dans le deuxième lemme, les hypothèses sur les conditions sont moins fortes alors que la difficulté augmente. 


\section{Un espace d'Asplund et ses propriétés topologiques}

Dans le troisième chapitre, nous fixons $\theta=\omega_{1}$ et $f=\min$ et nous obtenons un espace localement compact clairsemé $L_{1}$ de poids $\aleph_{1}$, de sorte que $C\left(K_{1}\right)$ est un espace d'Asplund non séparable, où $K_{1}=L_{1} \cup\{*\}$ est la compactification d'Alexandroff de $L_{1}$. Comme la fonction $f$ ici est simple et possède des propriétés fortes, nous obtenons les hypothèses du Lemme 2.13 et nous montrons le théorème suivant:

Théorème 1. Toute puissance finie de $K_{1}$ est héréditairement séparable.

Après, nous déduisons le résultat suivant à propos de l'espace de Banach $C\left(K_{1}\right)$ :

Théorème 2. $C\left(K_{1}\right)$ est un espace d'Asplund de densité $\aleph_{1}$ qui ne possède pas de système biorthogonal non dénombrable, ni de renormage Fréchet-différentiable.

Puis, nous modifions le Lemme 5.4 de Rabus [53] pour montrer la proposition suivante:

Proposition 1. Si $\left(\mu_{n}\right)_{n \in \mathbb{N}} \subseteq B_{C\left(K_{1}\right)^{*}}$ est une suite convergente vers $\delta_{*}$ dans la topologie faible-étoile, alors il existe $n_{0} \in \mathbb{N}$ tel que pour tout $n \in \mathbb{N}, n \geq n_{0}$, on a que $\mu_{n}(\{*\}) \neq 0$.

Finalement, nous utilisons cette proposition pour fournir un nouvel exemple d'un espace qui possède la propriété $(\mathrm{C})$ et qui ne possède pas la propriété (E): la propriété $(\mathrm{C})$ a été introduite par Corson dans [12]: un espace de Banach a la propriété (C) si toute famille d'ensembles convexes et fermés dont l'intersection est vide possède une sous-famille dénombrable dont l'intersection est vide; la propriété (E) a été introduite par Efremov dans [18]: on dit qu'un espace de Banach $X$ possède la propriété (E) si pour tout ensemble convexe et borné $C$ de $X^{*}$, si $\varphi \in \bar{C}^{w^{*}}$, alors il existe une suite $\left(\varphi_{n}\right)_{n \in \mathbb{N}} \subseteq C$ telle que $\left(\varphi_{n}\right)_{n \in \mathbb{N}}$ converge vers $\varphi$ dans la topologie faible-étoile. On montre, en effet, le résultat suivant:

Théorème 3. $C\left(K_{1}\right)$ possède la propriété $(C)$ de Corson et ne possède pas la propriété (E) de Efremov.

Le premier exemple d'un espace qui possède la propriété $(\mathrm{C})$ et qui ne possède pas la propriété (E) est une modification (non publiée) dûe à Justin T. Moore de l'espace d'Ostaszewski [49] (qui suppose le principe $\diamond$ ). 


\section{Un espace d'Asplund qui ne possède pas de système bior- thogonal non dénombrable}

Dans le quatrième chapitre, nous fixons $\theta=\omega_{2}$ et $f:\left[\omega_{2}\right]^{2} \rightarrow\left[\omega_{2}\right]^{<\omega}$ telle que pour tous $\xi<\eta<\theta, f(\{\xi, \eta\}) \subseteq \xi$ et si $A$ est une famille non dénombrable de sous-ensembles finis de $\omega_{2}$ qui forment un $\Delta$-système, alors il existe $a, b \in A$ et une bijection $e: a \rightarrow b$ qui préserve l'ordre qui est constante sur $a \cap b$ et tels que pour tout $\xi \in a, \xi \leq e(\xi)$ et pour tout $\zeta \in a \cap b$, tout $\xi \in a \backslash b$ et tout $\eta \in b \backslash a$ :

(i) si $\zeta<\xi$, alors $f(\{\zeta, \eta\}) \subseteq f(\{\xi, \eta\})$;

(ii) si $\zeta<\eta$, alors $f(\{\zeta, \xi\}) \subseteq f(\{\xi, \eta\})$;

(iii) $a \cap \xi \cap \eta \subseteq f(\{\xi, \eta\})$.

La consistance de l'existence d'une telle fonction est un résultat non publié dû à Koszmider dont la preuve se trouve dans l'Annexe A. Nous obtenons un espace localement compact clairsemé $L_{2}$ de poids $\aleph_{2}$, de sorte que $C\left(K_{2}\right)$ est un espace d'Asplund non séparable, où $K_{2}=L_{2} \cup\{*\}$ est le compactifié d'Alexandroff de $L_{2}$. Notre but principal dans ce chapitre est d'analyser les propriétés des espaces $K_{2}$ et $C\left(K_{2}\right)$.

Grâce aux propriétés de la fonction $f$, nous obtenons les hypothèses de l'autre lemme-clef prouvé dans le Chapitre 2 et nous montrons le résultat suivant:

Théorème 4. Toute puissance finie de $K_{2}$ est héréditairement séparable.

Puis, nous prouvons le fait suivant:

Théorème 5. La hauteur de $K_{2}$ est $\omega_{2}$.

Nous avons, donc, le premier exemple consistant d'un espace compact et clairsemé, héréditairement séparable dont la hauteur est $\omega_{2}$. Rappelons-nous que l'hypothèse du continu implique qu'il n'existe pas d'espace compact et clairsemé de hauteur $\omega_{2}$ et largeur dénombrable et Just [35] a utilisé le forcing de Cohen pour montrer que la non existence de tels espaces est consistante avec la négation de l'hypothèse du continu. Le premier exemple 
d'un espace compact et clairsemé dont la hauteur est $\omega_{2}$ et la largeur est dénombrable est celui construit par Baumgartner et Shelah [5].

Ensuite, nous déduisons le théorème suivant:

Théorème 6. $K_{2}$ est un espace tel que le degré de Lindelöf héréditaire de $K_{2}$ est strictement plus grand que le cardinal successeur de la densité héréditaire de $K_{2}$, c'est-à-dire, $h L\left(K_{2}\right) \not \leq$ $h d\left(K_{2}\right)^{+}$.

Ce résultat s'oppose au résultat qui assure l'inégalité duale, dû à Shapirovskiı̌ [62]: pour tout espace compact $K$, nous avons que $h d(K) \leq h L(K)^{+}$.

Finalement, nous déduisons quelques propriétés de l'espace de Banach $C\left(K_{2}\right)$ :

Théorème 7. $C\left(K_{2}\right)$ est un espace d'Asplund de densité $\aleph_{2}$ qui ne possède pas de systèmes biorthogonaux non dénombrables, ni de renormage Fréchet-différentiable.

Donc, $C\left(K_{2}\right)$ est le premier exemple d'un espace de Banach non séparable qui ne possède pas de systèmes biorthogonaux non dénombrables et dont la densité est $>\aleph_{1}$. Ce fait s'oppose au résultat suivant dû à Todorcevic [65]: tout espace $C(K)$ de densité $>\aleph_{1}$ possède un système semi-biorthogonal non dénombrable.

Ainsi, notre espace montre que ce résultat ne peut pas être généralisé, en remplaçant l'existence d'un système semi-biorthogonal non dénombrable par l'existence d'un système biorthogonal non dénombrable, ce qui répond à une question posée par Todorcevic.

\section{A. Morasses et la propriété $\Delta$}

Dans l'Annexe A, nous présentons la construction d'un forcing, qui utilise les morasses simplifiés de Velleman (voir [66] et [67]), qui force l'existence d'une fonction comme celle dont nous avons besoin au Chapitre 4: c'est un résultat non publié de Koszmider. 


\section{Referências Bibliográficas}

[1] P. Alexandroff, Über die Metrisation der im Kleinen kompakten topologischen Räume, Math. Ann. 92 (1924), no. 3-4, 294-301.

[2] E. Asplund, Fréchet differentiability of convex functions, Acta Math. 121 (1968), 31-47.

[3] J. Bagaria, Locally-generic Boolean algebras and cardinal sequences, Algebra Universalis 47 (2002), no. 3, 283-302.

[4] J. E. Baumgartner, Applications of the proper forcing axiom, Handbook of set-theoretic topology, North-Holland, Amsterdam, 1984, pp. 913-959.

[5] J. E. Baumgartner and S. Shelah, Remarks on superatomic Boolean algebras, Ann. Pure Appl. Logic 33 (1987), no. 2, 109-129.

[6] C. Brech, Aspectos combinatórios da geometria de espaços de Banach $C(K)$ com a propriedade de Grothendieck, Master's thesis, Universidade de São Paulo, 2004.

[7] - On the density of Banach $C(K)$ spaces with the Grothendieck property, Proc. Amer. Math. Soc. 134 (2006), no. 12, 3653-3663.

[8] K. Ciesielski, Set theory for the working mathematician, London Mathematical Society Student Texts, vol. 39, Cambridge University Press, Cambridge, 1997. 
[9] K. Ciesielski and R. Pol, A weakly Lindelöf function space $C(K)$ without any continuous injection into $c_{0}(\Gamma)$, Bull. Polish Acad. Sci. Math. 32 (1984), no. 11-12, 681-688.

[10] P. J. Cohen, The independence of the continuum hypothesis, Proc. Nat. Acad. Sci. U.S.A. 50 (1963), 1143-1148.

[11] _ The independence of the continuum hypothesis. II, Proc. Nat. Acad. Sci. U.S.A. 51 (1964), 105-110.

[12] H. H. Corson, The weak topology of a Banach space, Trans. Amer. Math. Soc. 101 (1961), 1-15.

[13] H. G. Dales and W. H. Woodin, An introduction to independence for analysts, London Mathematical Society Lecture Note Series, vol. 115, Cambridge University Press, Cambridge, 1987.

[14] R. Deville, G. Godefroy, and V. Zizler, Smooth bump functions and geometry of Banach spaces, Mathematika 40 (1993), no. 2, 305-321.

[15] _ Smoothness and renormings in Banach spaces, Pitman Monographs and Surveys in Pure and Applied Mathematics, vol. 64, Longman Scientific \& Technical, Harlow, 1993.

[16] J. Diestel and J. J. Uhl, Jr., Vector measures, American Mathematical Society, Providence, R.I., 1977, With a foreword by B. J. Pettis, Mathematical Surveys, No. 15.

[17] A. Dow and P. Simon, Thin-tall Boolean algebras and their automorphism groups, Algebra Universalis 29 (1992), no. 2, 211-226.

[18] N. M. Efremov, A condition for a Banach space to be a dual, Izv. Vyssh. Uchebn. Zaved. Mat. (1984), no. 4, 11-13. 
[19] R. Engelking, General topology, second ed., Sigma Series in Pure Mathematics, vol. 6, Heldermann Verlag, Berlin, 1989, Translated from the Polish by the author.

[20] M. Fabian, P. Habala, P. Hájek, V. Montesinos Santalucía, J. Pelant, and V. Zizler, Functional analysis and infinite-dimensional geometry, CMS Books in Mathematics/Ouvrages de Mathématiques de la SMC, 8, Springer-Verlag, New York, 2001.

[21] R. Fry and S. McManus, Smooth bump functions and the geometry of Banach spaces: a brief survey, Expo. Math. 20 (2002), no. 2, 143-183.

[22] J. R. Giles, D. A. Gregory, and B. Sims, Characterisation of normed linear spaces with Mazur's intersection property, Bull. Austral. Math. Soc. 18 (1978), no. 1, 105-123.

[23] A. S. Granero, M. Jiménez-Sevilla, and J.-P. Moreno, Intersections of closed balls and geometry of Banach spaces, Extracta Math. 19 (2004), no. 1, 55-92.

[24] R. Haydon, A nonreflexive Grothendieck space that does not contain $l_{\infty}$, Israel J. Math. 40 (1981), no. 1, 65-73.

[25] _ A counterexample to several questions about scattered compact spaces, Bull. London Math. Soc. 22 (1990), no. 3, 261-268.

[26] _ Trees in renorming theory, Proc. London Math. Soc. (3) 78 (1999), no. 3, $541-584$.

[27] R. Hodel, Cardinal functions. I, Handbook of set-theoretic topology, North-Holland, Amsterdam, 1984, pp. 1-61.

[28] M. Hrušák, P. J. Szeptycki, and Á. Tamariz-Mascarúa, Spaces of continuous functions defined on Mrówka spaces, Topology Appl. 148 (2005), no. 1-3, 239-252. 
[29] J. E. Jayne and C. A. Rogers, Borel selectors for upper semicontinuous set-valued maps, Acta Math. 155 (1985), no. 1-2, 41-79.

[30] T. Jech, Set theory, Springer Monographs in Mathematics, Springer-Verlag, Berlin, 2003, The third millennium edition, revised and expanded.

[31] M. Jiménez Sevilla and J.-P. Moreno, Renorming Banach spaces with the Mazur intersection property, J. Funct. Anal. 144 (1997), no. 2, 486-504.

[32] I. Juhász, K. Kunen, and M. E. Rudin, Two more hereditarily separable non-Lindelöf spaces, Canad. J. Math. 28 (1976), no. 5, 998-1005.

[33] I. Juhász and L. Soukup, How to force a countably tight, initially $\omega_{1}$-compact and noncompact space?, Topology Appl. 69 (1996), no. 3, 227-250.

[34] I. Juhász and W. Weiss, On thin-tall scattered spaces, Colloq. Math. 40 (1978/79), no. $1,63-68$.

[35] W. Just, Two consistency results concerning thin-tall Boolean algebras, Algebra Universalis 20 (1985), no. 2, 135-142.

[36] P. Koepke and J. C. Martínez, Superatomic Boolean algebras constructed from morasses, J. Symbolic Logic 60 (1995), no. 3, 940-951.

[37] P. Koszmider, On a problem of Rolewicz about Banach spaces that admit support sets, preprint.

[38] _ Forcing minimal extensions of Boolean algebras, Trans. Amer. Math. Soc. 351 (1999), no. 8, 3073-3117. 
[39] _ Banach spaces of continuous functions with few operators, Math. Ann. 330 (2004), no. 1, 151-183.

[40] _ A space $C(K)$ where all nontrivial complemented subspaces have big densities, Studia Math. 168 (2005), no. 2, 109-127.

[41] K. Kunen, Set theory. an introduction to independence proofs, Studies in Logic and the Foundations of Mathematics, vol. 102, North-Holland Publishing Co., Amsterdam, 1980.

[42] E. B. Leach and J. H. M. Whitfield, Differentiable functions and rough norms on Banach spaces, Proc. Amer. Math. Soc. 33 (1972), 120-126.

[43] S. Mazur, Über schwache Konvergenz in den Raumen $L^{p}$, Studia Math. 4 (1933), 129 133.

[44] S. Mazurkiewicz and W. Sierpiński, Contribution a la topologie des ensembles denombrables, Fund. Math. 1 (1920), 17-27.

[45] R. E. Megginson, An introduction to Banach space theory, Graduate Texts in Mathematics, vol. 183, Springer-Verlag, New York, 1998.

[46] I. Namioka and R. R. Phelps, Banach spaces which are Asplund spaces, Duke Math. J. 42 (1975), no. 4, 735-750.

[47] S. Negrepontis, The Stone space of the saturated Boolean algebras, Trans. Amer. Math. Soc. 141 (1969), 515-527.

[48] _ Banach spaces and topology, Handbook of set-theoretic topology, NorthHolland, Amsterdam, 1984, pp. 1045-1142. 
[49] A. J. Ostaszewski, A countably compact, first-countable, hereditarily separable regular space which is not completely regular, Bull. Acad. Polon. Sci. Sér. Sci. Math. Astronom. Phys. 23 (1975), no. 4, 431-435.

[50] A Plichko, A Banach space without a fundamental biorthogonal system, Dokl. Akad. Nauk SSSR 254 (1980), no. 4, 798-802.

[51] A. Plichko and D. Yost, Complemented and uncomplemented subspaces of Banach spaces, Extracta Math. 15 (2000), no. 2, 335-371, III Congress on Banach Spaces (Jarandilla de la Vera, 1998).

[52] R. Pol, A function space $C(X)$ which is weakly Lindelöf but not weakly compactly generated, Studia Math. 64 (1979), no. 3, 279-285.

[53] M. Rabus, An $\omega_{2}$-minimal Boolean algebra, Trans. Amer. Math. Soc. 348 (1996), no. 8, $3235-3244$.

[54] M. Rajagopalan, A chain compact space which is not strongly scattered, Israel J. Math. 23 (1976), no. 2, 117-125.

[55] F. Riesz, Sur les opérations fonctionelles linéaires, C. R. Acad. Sci. Paris 149 (1909), 974-977.

[56] J. Roitman, Basic $S$ and L, Handbook of set-theoretic topology, North-Holland, Amsterdam, 1984, pp. 295-326.

[57] _ Superatomic Boolean algebras, Handbook of Boolean algebras, Vol. 3, NorthHolland, Amsterdam, 1989, pp. 719-740. 
[58] S. Rolewicz, On convex sets containing only points of support, Comment. Math. Special Issue 1 (1978), 279-281, Special issue dedicated to Władysław Orlicz on the occasion of his seventy-fifth birthday.

[59] H. P. Rosenthal, The Banach spaces $C(K)$, Handbook of the geometry of Banach spaces, Vol. 2, North-Holland, Amsterdam, 2003, pp. 1547-1602.

[60] Z. Semadeni, Banach spaces of continuous functions. Vol. I, PWN-Polish Scientific Publishers, Warsaw, 1971, Monografie Matematyczne, Tom 55.

[61] N. A. Shanin, A theorem from the general theory of sets, C. R. (Doklady) Acad. Sci. URSS (N.S.) 53 (1946), 399-400.

[62] B. È. Shapirovskiǔ, Cardinal invariants in compacta, Seminar on General Topology, Moskov. Gos. Univ., Moscow, 1981, pp. 162-187.

[63] S. Shelah, Proper forcing, Lecture Notes in Mathematics, vol. 940, Springer-Verlag, Berlin, 1982.

[64] M. H. Stone, Applications of the theory of boolean rings to general topology, Trans. Amer. Math. Soc. 41 (1937), no. 3, 375-481.

[65] S. Todorcevic, Biorthogonal systems and quotient spaces via Baire category methods, Math. Ann. 335 (2006), no. 3, 687-715.

[66] D. Velleman, Morasses, diamond, and forcing, Ann. Math. Logic 23 (1982), no. 2-3, 199-281 (1983).

[67] _ _ Simplified morasses, J. Symbolic Logic 49 (1984), no. 1, 257-271. 
[68] K. Weierstrass, Über die analytische Darstellbarkeit sogenannter willkürlicher Functionen einer reellen Veränderlichen, Sitzungsberichte der Königlich Preussischen Akademie der Wissenschaften zu Berlin (1885), no. II, 633-639, 789-805.

[69] V. Zizler, Nonseparable Banach spaces, Handbook of the geometry of Banach spaces, Vol. 2, North-Holland, Amsterdam, 2003, pp. 1743-1816. 


\section{Índice Remissivo}

$C(K), 35$

$C_{0}(L), 35$

$K_{1}, 61$

$K_{2}, 72$

$L(X), 27$

$L_{1}, 61$

$L_{2}, 71$

$X^{\prime}, 29$

$X^{(\alpha)}, 29$

$\Delta$, propriedade, 40

$\Delta$-sistema, 25

lema do, 26

$d(X), 26$

$h L(X), 27$

$h d(X), 27$

$h t(K), 29$

$w(X), 26$

$w d(K), 29$

(C), propriedade, 68
(E), propriedade, 69

bump, função, 32

Alexandroff, compactificação de, 26

altura, 29

Asplund, espaço de, 32

biortogonal, sistema, 34

$\operatorname{Bor}(\mathrm{X}), 28$

Cantor-Bendixon, teorema de, 29

compactificação de Alexandroff, 26

conjunto derivado, 29

densidade, 26

hereditária, 27

derivado, conjunto, 29

Dirac, medida de, 28

disperso, espaço, 28

domínio de $p, 39$

espaço

de Asplund, 32 
disperso, 28

topológico genérico, 42

zero-dimensional, 26

Fréchet-diferenciável, função, 32

função

bump, 32

Fréchet-diferenciável, 32

grau de Lindelöf, 27

hereditário, 27

hereditária, densidade, 27

hereditário, grau de Lindelöf, 27

hereditariamente Lindelöf, 27

hereditariamente separável, 27

largura, 29

lema do $\Delta$-sistema, 26

Lindelöf, 27

Lindelöf hereditário, grau de, 27

Lindelöf, grau de, 27

$\mathrm{M}(\mathrm{K}), 28$

Mazur, propriedade de intersecção de, 33

medida

atômica, 31

de Dirac, 28 de Radon, 28

Namioka-Phelps, teorema de, 36

norma Fréchet-diferenciável, 32

pântano simplificado, 80

peso, 26

propriedade

$\Delta, 40$

(C), 68

(E), 69

de intersecção de Mazur, 33

Radon, medida de, 28

raiz do $\Delta$-sistema, 25

representação de Riesz, teorema de, 35

Riesz, teorema de representação de, 35

semi-biortogonal, sistema, 33

separável, 26

seqüência separada

à direita, 27

à esquerda, 27

sistema

biortogonal, 34

semi-biortogonal, 33

Stone-Weierstrass, teorema de, 35 
teorema

de Cantor-Bendixon, 29

de Namioka-Phelps, 36

de representação de Riesz, 35

de Stone-Weierstrass, 35

vizinhança genérica, 42

zero-dimensional,espaço, 26 